\title{
Clinical Relevance of Drug Interactions in HIV-Infected Patients Receiving Antiretroviral Therapy
}

\author{
Pedro Amariles ${ }^{1}$, Newar Giraldo Alzate ${ }^{1}$ and Maria Jose Faus ${ }^{2}$ \\ 1 University of Antioquia, Medellin, \\ 2University of Granada, Granada, \\ ${ }^{1}$ Colombia \\ 2Spain
}

\section{Introduction}

The identification, prevention, and clinical solution of drug interactions (DIs) are a critical aspect to achieve desired pharmacotherapy goals in patients infected with human immunodeficiency virus and/or affected by acquired immunodeficiency syndrome (HIV/AIDS) receiving antiretroviral (ARV) therapy, mainly because DIs may lead that ARV therapy will be unsafe and/or ineffective and thus, DIs may be clinical relevant. Additionally, in this group of patients the DIs are more frequent among other aspects by:

- $\quad$ The use of Highly Active Antiretroviral Therapy (HAART) or combined Antiretroviral Therapy (cART) includes three or more ARV drugs; (DHHS, 2011) therefore it is associated with a greater likelihood of DIs.

- The pharmacokinetic properties of ARV drugs, for instance several of them are metabolized through complimentary cytochrome P450 isoenzymes, thus their therapeutic use could be accompanied by frequent DIs. (Miller et al., 2007)

- $\quad$ ARV drugs are concurrently used with other class of medications for other common conditions, mainly infections and cardiovascular disease; and many of these medications used to treat these conditions are metabolized through complimentary cytochrome P450 isoenzymes, so several pharmacokinetic DIs may occur.

Accordingly, some studies illustrate that the $96 \%$ of patients receiving HAART or cART has at least a clinical condition or use a concomitant drug that could cause that ARV therapy may be unsafe (adverse drug reactions) or ineffectiveness (therapeutic failure). (Grimes et al., 30 2002) Therefore, identifying, preventing, and solving clinically relevant DIs is recognized both as a topic of great importance in achieving therapeutic goals for drug therapy (Kashuba, 2005) as a constant challenge to health care providers to HIV-infected patients receiving HAAR or cART. In addition, the clinical significance of a DI depends on the disposition and toxicity profile of the drug being administered. Thus, in HIV-infected patients assessing the clinical relevance of a DI is complex due to the large interpatient variability in pharmacokinetics exhibited by most ARV drugs, and then the evaluation and prediction of clinical effect of a DI is critical in the pharmacotherapy of patients with HIV/AIDS. 
Since most of ARV drug DIs are clinically relevant, it is considered appropriate both to outline the concept, types, mechanisms, and effects of ARV DIs on drug therapy, and to present a comprehensive summary of those drugs that are affected and the clinical relevance of ARV DIs. In this way, the aim of this chapter is provide evidence and systematize information about DIs in HIV-infected receiving ART therapy, which allow define, evaluate, and predict the clinical relevance of the DIs, highlight those associated to pharmacokinetic mechanism. In this way, a proposal to identify, evaluate, and predict DIs considered as clinically relevant is presented, in which clinical relevance of a DI is defined according to the probability of their occurrence and to the severity of clinical effect in patient health (adverse event or therapeutic failure). (Amariles et al., 2007a)

Previous review about DIs with ARV, (Amariles et al., 2007b; Giraldo et al., 2010) achieved as a result of searched in Pubmed/Medline database, have showed that, in the case of clinically relevant pharmacokinetic interactions, nearly $80 \%$ are related to changes in systemic clearance, mainly associated to the systemic inhibition or induction of the metabolic activity of the cytochrome P-450 (CYP-450), mostly CYP3A4 isoform, whereas approximately $15 \%$ are related to changes in bioavailability (changes in gastrointestinal $\mathrm{pH}$, presystemic clearance [mediated by CYP3A4 hepatic or intestinal]) or in P-glycoprotein activity). (Amariles et al., 2007b; Giraldo et al., 2010)

For this chapter, the earlier published information (Amariles et al., 2007a, 2007b; Giraldo et al., 2010; Amariles, 2002.) have been complement with information achieved from both a structured and systematic review of publications on Pubmed/Medline and references cited in relevant articles, and in other electronic databases (SIETES, MEDSCAPE, and TRIPDATABASE), and supplemented by other primary and secondary information sources to identify DIs in HIV-infected patients. Thus, searched MeSH terms were drug interactions, antiretroviral agents (or drugs), drug food interactions, drug nutrient interactions, drug laboratory test interference, drug in special situations (age, diseases), drug herbal plant interactions, computerized drug interactions, decision clinical computer based, and clinical relevance, clinically relevant or significantly relevant.

Finally, according with clinical relevance of the DIs, pairs of the identified DIs have been classified in four levels, according to rate probability and severity, (Amariles et al., 2007a, 2007 b; Giraldo et al., 2010) and then, the different drug pairs have been structured in a software designed to facilitate the identification, evaluation, and prediction of clinical relevant DIs. Current, 1,082 drug pairs of potential DIs have been identified, near to $80 \%$ of them due to pharmacokinetic mechanism (changes in plasma concentration), mainly associated to systemic enzyme inhibition. The scaling of these 1,082 drug pairs of recognized DIs, according to different dosage forms and strengths of identified drugs, generates a total of 6,087 pairs of DIs, in which, according their clinical relevance, 4,158 (68.3\%) are clinical relevant (Levels 1 and 2) in HIV-infected patients receiving ARV therapy. Thus, the designed software meets the requirements defined for this type of program (Gaikwad et al., 2007; Rodríguez et al., 2009) and most important it facilitates the assessment, prediction, and decision on clinical relevance of 4,158 ARV DIs, which are considered of clinical interest in patients with HIV/AIDS (levels 1 and 2).

\section{Drug interactions in HIV-infected patients receiving antiretroviral therapy}

\subsection{Concept, type, and mechanism of drug interactions}

Concept of DI. In patients with HIV/AIDS a DI could be assumed as non-therapeutic and quantitative modification in the magnitude or duration of the drug effect (decrease the 
efficacy or increase the toxicity) that may lead to therapeutic failure or adverse drug reactions associated to a previous or a concomitant use of another drug (drug-drug interactions), including herbal drug products (herbal-drug interactions), certain type of food (drug-food interactions) or due to a patient's physio-pathological condition (drugdisease interaction). (Amariles et al., 2007a) Additionally, changes on the results of certain laboratory tests that may produce some drugs (drug - laboratory tests interactions) (Maddox et al., 1980) or on the bioavailability of several nutrients (drug - nutrient interactions) (Chan, 2002; Santos \& Boullata, 2005) may be considered as a DI. (Amariles, 2002)

Elsewhere, the increasing use of herbal products worldwide and the growth of the herbal product industry have led to rising the identification and characterization of clinically relevant DIs among several drugs with some of these products, for instance St. John's Wort (Hypericum Perforatum L.), which has been the characterization of another type of DI: herbal-drug drug interactions. (Markowitz \& DeVane, 2001)

Although from a pharmacological perspective, some DIs may lead to a required therapeutic effect, for instance "pharmacokinetic enhancement or ritonavir boosting, strategy in which low doses of ritonavir -100 to $200 \mathrm{mg}$ - (a cytochrome P4503A inhibitor) are used in combination with other protease inhibitors to increase antiretroviral drug exposure (Rathbun \& Rossi, 2002), from a risk perspective, the efforts should focus on evaluating, predicting, and solving DIs with high probability to produce effects that are undesirable and to arise toxicities or therapeutic failures, which are termed as clinically relevant drug interactions. (Amariles, 2002; Amariles et al., 2007a)

Pharmacological mechanism of drug interactions. The previous or concomitant use of a drug, herbal drug product, or food (like a patient's physio-pathological condition) may cause a non-therapeutic and quantitative modification in the magnitude or duration of the drug effect because such substance or situation causes an alteration that involve one or more of the three pharmacologic processes namely biopharmaceutics, pharmacokinetics, or pharmacodynamics.

The biopharmaceutics is relating both to factors that influence the drug release from a drug product and the drug dissolution rate in the absorption site. Whereas, the pharmacokinetics is relating to drug absorption, distribution, metabolism, and excretion (ADME) (what the body does to the drug). As most drugs proceed through first order kinetics, and the process of ADME usually follows first order kinetics as well, the relationship between dose/time and drug plasma concentration for most drugs is linear. Thus, the biopharmaceutics and pharmacokinetics phases determine the drug plasma concentration. Similarly, since the relationship between plasma concentration and drug available on the site of action or biophase is linear, and the concentration on biophase is directly related to the intensity and duration of therapeutic response for most drugs as well, the relationship between drug plasma concentration and the magnitude of drug effect for most drugs is linear (figure 1).

Pharmacodynamics phase (what the drug does to the body): the pharmacodynamics studies the mechanism of action (specific molecular drug-target interaction, usually as a result of binding to a receptor or an enzyme, through which a drug causes its pharmacological response or effect (pharmacological surrogate or clinical effect as result of drug- target interaction). 


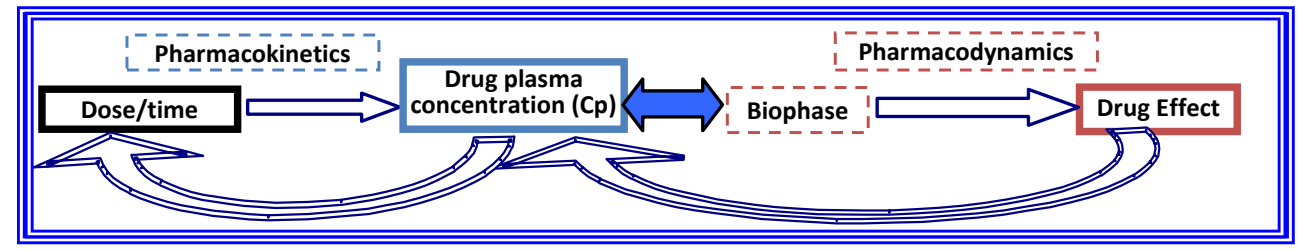

Fig. 1. Pharmacokinetics, plasmatic concentration (Cp), and pharmacologic effect.

Based on the pharmacological mechanism that explains the non-therapeutic and quantitative modification in the magnitude or duration of the drug effect, the DI may be classified as:

- Pharmacokinetic DI: The non-therapeutic and quantitative modification in the magnitude or duration of the drug effect is explained mainly by a change in the drug plasma concentration associate with substance or situation that causes the DI. The change in the drug plasma concentration may be attributed to a biopharmaceutic (drug release and dissolution) and/or pharmacokinetic (ADME) alteration. Thus, biopharmaceutic DIs are included in pharmacokinetic DIs (both biopharmaceutic and pharmacokinetic DIs are attributed to a change in the drug plasma concentration).

- Pharmacodynamic DI: The non-therapeutic and quantitative modification in the magnitude or duration of the drug effect occurs without change in the drug plasma concentration. This type of DIs are mainly due to drugs or substances that have either similar (synergism) pharmacological effect or opposing (antagonistic) pharmacological effect or physio-pathological condition (drug-disease interactions) that contributes or facilities (synergism) the therapeutic or toxic effect of the drug, or that diminish or counteract its therapeutic effect (antagonism). In general, in one patient, the use of drugs those have a similar unsafe profile increases the likelihood and severity of adverse drug effects, for instance the use of drug-induced hepatic or renal toxicity.

\subsection{Proposal to evaluate and predict the clinical relevance of pharmacokinetic drug interactions in HIV-infected patients receiving antiretroviral therapy (Amariles et al., 2007b)}

HAART or CART has improved survival of HIV-infected patients, but they currently have chronic co-morbidities which require pharmacologic interventions with several medications, increasing the risk of DIs. In clinical practice, it is known that DIs may lead important pharmacotherapy problems especially for illnesses which require using various medications. Therefore, clinically relevant DIs are frequent among HIV-infected patients who are receiving ARV therapy. (Miller et al., 2007) In this context, ARV drugs may lower the efficacy or enhance side effects or toxicity of several of these drugs, and similarly some of these drugs may cause therapeutic failure or increase the toxicity of ARV drugs. (Fletcher et al., 2000)

DIs are especially important for drugs with narrow therapeutic indices and may either be pharmacodynamic or pharmacokinetic in nature. However, pharmacokinetic DIs may be more frequent complex to evaluate and to predict the effect among HIV-infected patients receiving ARV therapy. For instance, protease inhibitors (PIs), non-nucleoside reverse transcriptase inhibitors (NNRTIs), and the CCR5 antagonist maraviroc are metabolized through the CYP450 system, mostly by CYP3A4. (DHHS, 2011) In addition, each of the NNRTIs and PIs induce and/or inhibit specific CYP450 enzymes and consequently are prone to cause pharmacokinetic DIs, (Pau \& Boyd, 2010) mainly when they are concomitant used in patients with HIV and 
with others important co-morbidities, such as dyslipidaemia, hypertension, tuberculosis, and opiate dependence, in which is needing to use several drugs that may be both substrate and selected inducers or inhibitors of CYP3A4. (Josephson, 2010)

In general, the clinical relevance of a pharmacodynamic DI can often be evaluated, predicted, and monitored easily, because the process is supported by knowledge of the drugs mechanism of action and pharmacological effects (therapeutic and adverse), complemented with the definition and monitoring of parameters related to the drug clinical effects (clinical effects and toxicity profile), if possible in a quantitative way. Whereas, the pharmacokinetic DIs (alterations in drug plasma concentration associated to changes in the release, dissolution, absorption, distribution, metabolism, or excretion of drug) are more complex and may not be as easily evaluated, predicted, and monitored as the pharmacodynamic DIs; thus, the process requires both knowledge of pharmacology, pharmacotherapy, and clinical expertise. Thus, it is important to present a development proposal, which have been adjusted with goal to evaluate and predict the clinical relevance of pharmacokinetic DIs in HIV-infected patients receiving ARV therapy. (Amariles et al., 2007b; Giraldo et al., 2010)

\subsubsection{Identifying and assessing if one of the medication that the patient is using (or} that the patient will be use) is considered as a drug with narrow therapeutic indices The therapeutic index of a drug is the ratio of the dose that produces toxicity (drug plasma concentration that elicits the toxic effect in 50 percent of treated individuals $-\mathrm{TD}_{50^{-}}$), and the dose that produces a clinically desired or effective response in a population of individuals (drug plasma concentration that elicits the therapeutic effect in $\mathbf{5 0}$ percent of the treated individuals $-E^{-} D_{50^{-}}$) as shown in equation 1 (Katzung, 2009)

$$
\text { Equation 1. Index therapeutic }=\mathrm{TD}_{50} / \mathrm{ED}_{50}
$$

Both $\mathrm{TD}_{50}$ and $\mathrm{ED}_{50}$ are calculated from dose response curves, which represent the frequency with which each drug plasma concentration elicits the therapeutic effect or the toxic effect in the population (figure 2).

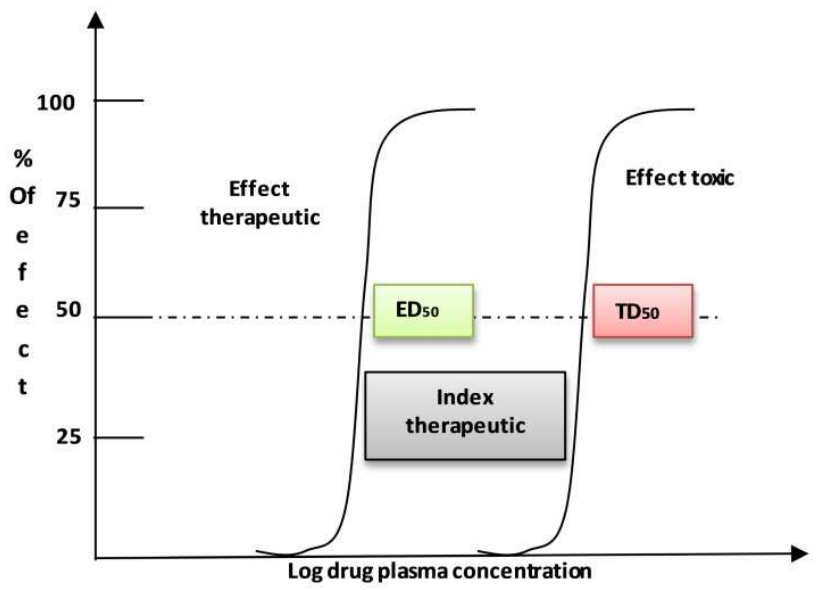

Fig. 2. Dose-responsive curves and Therapeutic Index 
From a clinical perspective, drug therapeutic range corresponds at drug plasma concentrations associate to likelihood of achieving, in the most patients, the maximum therapeutic effect with the minimum toxic effect. The probability that a pharmacotherapy process will be effective and safe increases if both the maximum drug plasma concentration and the minimum drug plasma concentration of the steady state associate to a specific dosing schedule in a patient are included within the therapeutic range (population) or therapeutic index (individual) of the drug (minimum effective level and minimum toxic level, which are theoretical). If the concentrations achieved at steady state are outside of the therapeutic range or index (excluded), it is increases the probability of drug failure or ineffective (if the concentration is lesser than the minimum effective level) or of adverse or toxic effects (if the concentration is higher than the minimum toxic level). Therefore, drug therapy should obtain that the achieved drug plasma concentrations at steady state are included within the therapeutic range or index (theoretical) is a specific patient, as shown in figure 3.

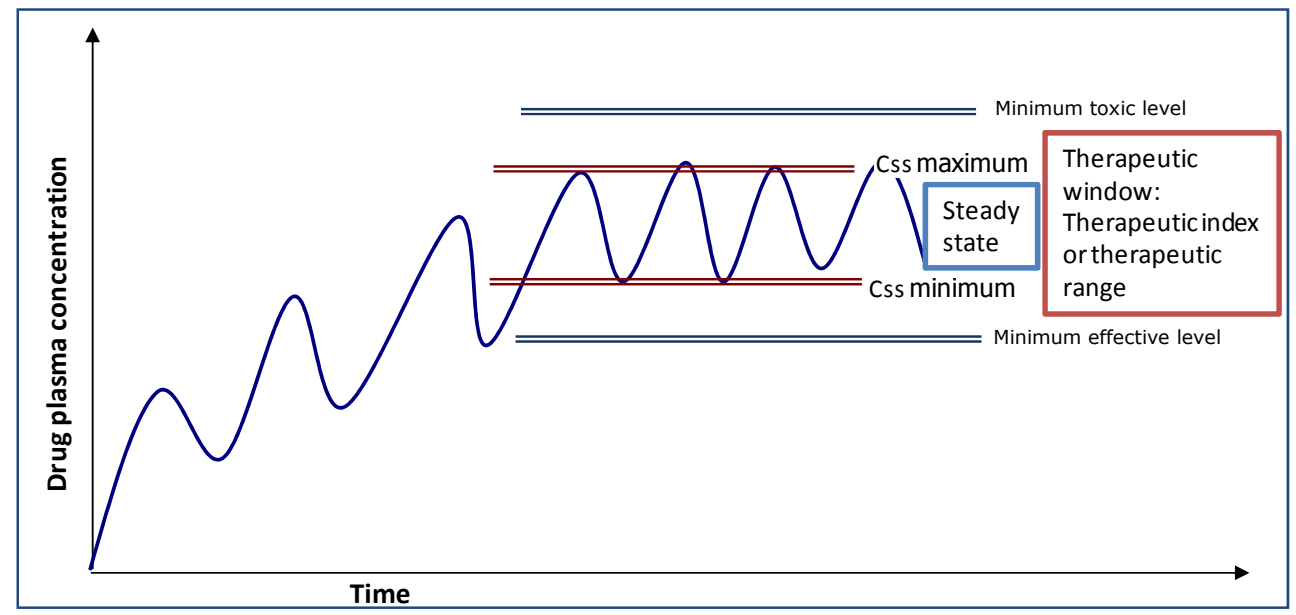

Fig. 3. Relationship between drug plasma concentrations achieved in steady state and the theoretical therapeutic window (therapeutic index or therapeutic margin)

The probability that a pharmacokinetic DI causes that the drug achieved drug plasma concentrations at steady state are outside of the therapeutic range or index is inversely proportional to the difference between minimum effective level and minimum toxic level that are defined in the therapeutic index or range of the drug. The probability is higher (increased) for drugs with small difference between the minimum effective concentrations and the minimum toxic concentrations (drugs with a narrow therapeutic range or index).

It could be practice to consider a narrow therapeutic range or index drug if the drug: (FDA, 2005) (1) Require pharmacokinetics (therapeutic drug concentration) or pharmacodynamic (measuring clinical effects) monitoring, thus the effective and safe use of the drug require careful dosage titration and patient monitoring; (2) have less than a 2-fold difference in median lethal dose $\left(\mathrm{LD}_{50}\right)$ and median effective dose $\left(\mathrm{ED}_{50}\right)$ values; or $(3)$ have less than a 2fold difference in the minimum toxic plasma concentrations and the minimum effective plasma concentrations

From a clinical practical perspective, there are lists of narrow therapeutic index drugs, including their minimum effective concentrations and the minimum toxic concentrations, 
which include drug as digoxin, lithium, vancomycin, aminoglycosides, and anticonvulsants. Although it is knowing that some drugs has narrow therapeutic index, for instance oral anticoagulants and insulin, they may not be included in this kind of lists, mainly due to: (1) Absence of population data of minimum toxic concentrations and minimum effective concentrations; (2) presence of a large interindividual pharmacodynamic variability (in some patient a similar drug plasma concentration may cause different magnitude or duration of the clinical effects); and (3) there are not a reasonable relationship between drug plasma concentrations and clinical effects. For that reason, although data for dose-response curves is obtained from several individuals, the therapeutic index is assumed more as an individual perspective whereas therapeutic range is assumed more as a population perspective.

In drug therapy process using drugs with a narrow therapeutic range or index, pharmacokinetic DIs may cause an increase in blood/serum concentrations and thus could lead to drug toxic effects, or may cause a decrease in blood/serum concentrations and thus could lead to drug failure or ineffectiveness. Therefore, in the process of evaluate and predict the clinical relevance of a DI, the first stage is to establish whether any drugs that patient is using (or that will use) is considered as a narrow therapeutic range or index drug. In general, if the drugs used have a broad therapeutic index or margin, the probability of a pharmacokinetic DI makes that the drug be unsafe or ineffective is low, because the probability of a change leads to achieved drug plasma concentrations are excluded at the therapeutic range or index (below of the minimum effective concentrations or above of the minimum toxic concentrations) is minimum. In general, if pharmacokinetic DIs (as well as pharmacodynamic DIs) involves drug products that are not considered as narrow therapeutic range or index drugs they will be clinical irrelevant; therefore in these cases the evaluating and predicting of DI may not be needed.

ARV drugs as Narrow Therapeutic or Range Index Drugs. Due to their pharmacological and clinical features, ARV drugs may be considered as narrow therapeutic or range index drugs. (FDA, 2005) For instance, although there are some controversies due to broad intraand inter-individual pharmacokinetic variability of ARV drugs, (Nettles et al., 2006) PIs and NNRTIs have defined drug plasma concentrations related to maximum efficacy and safety (therapeutic range) and, therefore ARV drugs are susceptible to therapeutic drug monitoring (assessing and monitoring drug concentration) (Justesen, 2006; Wertheimer et al., 2006), as shown in table 1 . As consequence, it is possible to establish, that patients that are receiving PI/NNRT have a high susceptibility to present clinically relevant DIs. Additionally, some medications used in HIV-infected patients for treatment or prevention of some chronic co-morbidities and opportunistic infections may be considered as narrow therapeutic range or index drugs, for instance rifampin, rifabutin and other antibiotics, anticonvulsants, statins, antidepressants, antihypertensives, and opioids (DHHS, 2011).

\subsubsection{Identifying and predicting consequences of drug interaction on the pharmacokinetics, plasma concentrations, and affected drug clinical effects (step more complex)}

The consequences of a pharmacokinetic DI on the main pharmacokinetic process, on the drug plasma concentrations, and thus on the affected drug clinical effects, depends among other aspects, of: (1) the magnitude that the respective pharmacokinetic process affects 


\begin{tabular}{|l|l|}
\hline Drug & Therapeutic range or index $\mathbf{( n g} / \mathbf{m L})$ \\
\hline Saquinavir & $250-600$ \\
\hline Ritonavir (solo) & $150-2.100$ \\
\hline Indinavir & $100-1.000$ \\
\hline Nelfinavir & $800-3.000$ \\
\hline Amprenavir & $400-2.200$ \\
\hline Lopinavir/ritonavir & $1.000-9.000$ \\
\hline Atazanavir & $150-1.000$ \\
\hline Tipranavir & $6.500-50.000$ \\
\hline Nevirapine Cmin & 3.500 \\
\hline Efavirenz & $1.000-4.000$ \\
\hline Delavirdine & Limited information \\
\hline
\end{tabular}

aAssessment as its active metabolite (m8). Cmin: minimum concentration

Table 1. Drug plasma concentrations related to effective minimum level and toxic minimum level that defined the therapeutic index or range of ARV drugs. (Justesen, 2006; Nettles et al., 2006; Wertheimer et al., 2006)

achieved drug plasma concentrations with the dosing-schedule used in the patient; and (2) the magnitude of change that the DI causes on altered pharmacokinetic process. These two aspects determine the influence of a DI both on the magnitude of change on drug plasma concentrations and on the probability of achieved drug plasma concentrations at steady state are excluded from the therapeutic range or index. Relate to this issue, the average steady-state concentration (Cpss) is an excellent estimator of achieved drug plasma concentrations, which is determined by the ratio between the drug delivery rate (input rate, which depends directly on the dose -D-, and the bioavailability -F-, and inversely on the dosing interval -T-), and the clearance rate-CL- (output rate), as shown in equation 2.

$$
\text { Equation 2. Cpss }=\frac{\mathbf{D} \mathbf{x} \mathbf{F}}{\boldsymbol{\tau} \mathbf{X} \mathbf{C L}}
$$

Bioavailability (F). Although, bioavailability of drug is classically defined as the rate and extent to which the active ingredient (drug substance) is absorbed from the dosage form (upon oral administration), from a clinical perspective, this pharmacokinetic parameter may be assumed as the amount of active ingredient (drug substance) from the dosage form (drug product, upon oral administration) that reaches the systemic circulation unchanged. Therefore, the bioavailability of drug is influenced by: (1) the disintegration of the dosage form, the release of drug from a drug product, and the dissolution of drug in the absorption site; (2) the presystemic metabolism both in the gut lumen (extrahepatic) and in the liver by CYP450, especially CYP3A4; and (3) the contribution of intestinal transporters, which may decrease the bioavailability, by efflux effect of intestinal P-glycoprotein (P-gp) in drug absorbed from apical to basolateral), or may increase the bioavailability, by effect of the anionic organic polypeptide (TAOP), especially the type B. (Ho \& Kim, 2005)

Systemic or total clearance (CL) is defined as the volume of plasma in the vascular compartment cleared of drug per unit of time [volume/time], mainly by hepatic metabolism 
(hepatic clearance) or by renal excretion (renal clearance) but also by other ways, such as biliary excretion. CL is a measure of the efficiency of human organism to remove irreversibly a drug from the systemic circulation or bloodstream by all routes of elimination, mainly by biotransformation (or drug metabolism) and excretion.

Drugs are metabolized (changed) usually by enzymes found mainly in the liver but also in small intestine, lung, kidney, and skin to a metabolites by process known as drug metabolism or biotransformation. Biotransformation often changes no-polar or lipophilic drugs into metabolites more polar or hydrophilic, which tend to be excreted in the urine (renal excretion) or in the stool (biliary excretion) as glucuronate, sulphate or acetate conjugates. While, polar or hydrophilic drugs may be excreted without drug metabolism through renal excretion.

A renal pharmacokinetic DI may be clinically relevant if: (1) there is a competitive inhibition of tubular secretion of the drug and (2) the renal clearance contributes more than $30 \%$ and drug systemic clearance. (Bonate et al., 1998; Launay et al., 2006) Additionally, certain drugs, for instance ritonavir may inhibit the renal secretion of certain drugs, which may be critical for drugs that are mainly eliminated by this via. For example, when digoxin is used concomitant with ritonavir, the PI may lead to an increase in the levels and pharmacological effects of digoxin. (Ding et al., 2004)

Concepts provide above, particularly the equation 2, lead to understand why clinically relevant pharmacokinetic DIs are mainly explained by changes in systemic clearance and bioavailability. Thus, almost $80 \%$ of pharmacokinetic DIs are related to changes in systemic clearance, mainly associated to the systemic inhibition or induction of the metabolic activity of the CYP450 isoenzymes, and approximately 15\% related to changes in bioavailability (changes in gastrointestinal $\mathrm{pH}$, presystemic clearance [by hepatic or intestinal CYP3A4 isoenzyme]) or by in P-gp activity). (Amariles et al., 2007b; Giraldo et al., 2010)

The process of evaluating and predicting of the clinical relevance of a pharmacokinetic DI continues with the identification if the main route of drug elimination is hepatic $o$ renal, and thus if the drug is remove irreversibly from the systemic circulation or bloodstream by excretion renal or by hepatic metabolism. Generally, if the drug is eliminating by hepatic metabolism, the probability that a pharmacokinetic DI will be clinical relevant is elevated. In these cases, subsequent to identify whether the systemic elimination of any drugs that patient is using (or drugs that patient will use) occurs primarily by hepatic metabolism, the process must continue with the evaluation of the effect that may cause one possible hepatic metabolism inhibition or induction on drug plasma concentrations.

Because a near to $80 \%$ of clinically relevant pharmacokinetic DIs are related to hepatic metabolism, both systemic and presystemic, during the process of developing new drugs it is important to characterize and to predict DIs related to hepatic metabolism. With this goal, both cell cultures are used to establishing the ability of the new drug to modify the activity of major CYP450 isoenzymes, and the assessment of the susceptibility of the drug metabolism to be affected by drugs recognized as enzyme inhibitors and inducers. (Tucker, 2001; Obach et al., 2005, 2006)

The major route of elimination of PIs and NNRTIs is by hepatic metabolism and thus the pharmacokinetic DIs may be clinical relevant. Not at all, for nucleoside or nucleotide 
analogue reverse transcriptase inhibitors (NRTIs), due to they are eliminated primarily by renal excretion, clinically relevant pharmacokinetic DIs are less frequent. However, among NRTIs, abacavir is metabolized by alcohol dehydrogenase and zidovudine by gucoroniltransferase, which may cause that these two drugs to have interactions associated to changes in the activity of the respective enzymes. Additionally, there is evidence that tenofovir may modify the atazanavir metabolism and thus drug plasma concentrations and clinical effects of this PI; similarly, atazanavir and lopinavir/ritonavir may alter the drug plasma concentrations and clinical effects of tenofovir (see below). In addition, the systemic elimination of some drugs, considered as narrow therapeutic index or margin drugs, and commonly used in patients with HIV/AIDS (such as rifamycins, anticonvulsants, statins, and antidepressants) occurs by hepatic metabolism and, therefore, clinically relevant pharmacokinetic DIs are likely to arise.

\subsubsection{Evaluation of the effect that may cause one possible hepatic metabolism inhibition or induction on the plasma concentrations of the potentially affected drug}

The process of evaluation of a DI associated to hepatic metabolism needs: (1) identify the CYP450 enzyme which is responsible for the biotransformation of the drug whose metabolism can be altered, and (2) identifying agents that alter (induce or inhibit) the metabolic capacity of the CYP450 enzyme. The proper observance of this stage implies following three steps.

a. Identification of the CYP450 enzyme which is responsible for the biotransformation of the drug that may be altered. A detailed and updated list of major CYP450 isoenzymes, together with their most common substrates, inhibitors, and inducers can be found on the following web sites:

- $\quad$ http://medicine.iupui.edu/clinpharm/DDIs/ and

- $\quad$ http://medicine.iupui.edu/clinpharm/DDIs/ClinicalTable.asp

In the case of PIs and NNRTIs, their systemic metabolism occurs primarily by CYP3A4. Additionally, there are other drugs commonly administered to HIV/AIDS patients which are metabolized by CYP3 or CYP2 families.

b. Identifying drugs that may modify (inducing or inhibiting) the metabolic capacity of CYP2 and CYP3 families. For instance, rifampin, rifabutin, carbamazepine, phenobarbital, phenytoin, and Saint-John's-wort may induce the activity of CYP2 and CYP3 families, whereas azole antifungals, macrolides, calcium antagonists, immunosuppressants, and grapefruit juice may inhibit CYP3 family. Most the drugs ARV used in the treatment of HIV are metabolized by the CYP450 enzymes and they have the capacity to modify the activity of CYP3 family and, to a lesser extent, the activity of CYP2 family. Among the NNRTIs, efavirenz and nevirapine mainly induce CYP3A4 and CYP2B6, while delavirdine is primarily an enzymatic inhibitor of the CYP3A4, and etravirine inhibits CYP2C9 and CYP2C19 while inducing CYP3A4. However, efavirenz may inhibit the activity of CYP3A4, CYP2C9, and CYP2C19 isoenzymes. In vitro studies show most of the PIs are inhibitors of activity of CYP3A4 isoenzyme (atazanavir, darunavir/ritonavir, lopinavir/ritonavir, saquinavir, tipranavir/ritonavir) or strong CYP3A4 inhibitors (ritonavir, indinavir, nelfinavir). Some PIs both inhibit and induce CYP3A4 (amprenavir, fosamprenavir). Ritonavir also inhibits CYP2D6. The NRTIs, the fusion inhibitor enfuvirtide, and the integrase inhibitor raltegravir are not metabolized by the CYP450 system. 
In clinical practice, inhibition of hepatic CYP enzymes or metabolic inhibition is one of the most common DI mechanisms and it is usually reversible and competitive. Most pharmacokinetic DIs occur when a drug increases or decreases metabolism of other drugs in the liver (CYP enzymes or glucuronidation). When a drug or substance causes a decrease in the systemic hepatic metabolism and clearance of a drug, this substance generates an increase in plasma concentrations and may lead to emerge of adverse drug events or toxicities. Thus, the process of evaluating and predicting the effects of this kind of DI needs to identify the drugs that are strong or moderate inhibitors of activity of different CYP450 isozymes known also as "enzyme inhibitors for excellence".

c. Determining if the pharmacokinetic DI related to hepatic metabolism might be either one-way or two-way direction (bidirectional) and if it might affect the levels and effects of the two drugs involved; situation that is likely to occur, because the need to use simultaneously either inhibitor drugs (e.g., macrolides and antifungal azoles) or inducer drugs (for instance rifampicin and carbamazepine) together with PIs and/or NNRTIs is common.

\subsubsection{Evaluation of changes in the bioavailability of drugs orally administered}

Changes in presystemic metabolism (due to inhibition or induction of extra-hepatic or hepatic CYP3A subfamily), in the activity of P-gp, or in the gastrointestinal $\mathrm{pH}$ may affect both the amount absorbed and the effects of some ARV drugs. In turn, antiretroviral drugs, particularly PIs, may modify the bioavailability and effects of some drugs, mainly through inhibition of presystemic metabolism and, to a lesser extent, of the activity of P-gp. Didanosine, especially in its dosage form as buffer solution, can change gastrointestinal $\mathrm{pH}$ and the amount absorbed by some drugs.

For drugs with kinetics elimination of first order or linear (most drugs used at therapeutic doses, phenytoin is one important exception), as it may be deduced from the equation 2 , the increase or decreases of average steady-state concentration (Cpss) is inversely related to the decrease or increase of $C L$ (Cpss $\cong 1 / C L)$. Generally, for narrow range or index drugs, a pharmacokinetic DI may be clinically relevant, if the DI causes a change in the achieved Cpss of $+/-20 \%$ (FDA, 1999). As Cpss $\cong 1 / C L$, a decrease (associate to a reduction of hepatic metabolism) in the CL of $10 \%, 15 \%, 20 \%, 30 \%, 50 \%$, and $75 \%$ may produce an increase in the Cpss of $11 \%, 18 \%, 25 \%, 43 \%, 100 \%$, and $400 \%$, respectively. The expected increasing of Cpss is calculated from the ratio of 100 divided by 100 less the percentage that CL is decreasing [100/ (100 less \% of decrease of CL)]. Thus, if CL decreases in $10 \%$, the increase of $11 \%$ is obtained from the ratio of 100 divided by 100 less 10 (100/90), which result is $111 \%$ and it is showing that Cpss increases in $11 \%$. Similarly, $43 \%$ is obtained from the ratio of 100 divided by 100 less 30 (100/70) which result is $143 \%$ and it is showing that Cpss increases in $43 \%$; and so similarly for other values. (Amariles, 2002) In the situations of increases of hepatic metabolism (induction enzymatic), it is requiring a minimal increase of $25 \%$ in CL, which may be caused a decrease of $20 \%$ in the achieved Cpss.

The drug plasma concentration before of the pharmacokinetic DI is another factor that contributes significantly to the magnitude of change in the clinical effect. For example, if diltiazem may decreased CL of quinidine (therapeutic range: $1-4 \mu \mathrm{g} / \mathrm{mL}$ ) in 35\% (Laganiere et al., 1996), thus it may cause an increase of $54 \%$ in the achieved Cpss of quinidine. Thus, if the quinidine Cpss on time interaction was of $2.5 \mu \mathrm{g} / \mathrm{mL}$, it would increase to $3.85 \mu \mathrm{g} / \mathrm{mL}$, whereas if the quinidine Cpss was of $3.5 \mu \mathrm{g} \mathrm{mL}$, it would increase to $5.39 \mu \mathrm{g} / \mathrm{mL}$. As 
consequence, the probability of toxicity at $5.39 \mu \mathrm{g} / \mathrm{mL}$ (outside the therapeutic range) is higher than at $3.85 \mu \mathrm{g} / \mathrm{mL}$ (within the therapeutic range). (Amariles, 2002)

2.3 Determining and predicting of the clinical relevance level (Amariles et al., 2007a) A relevance analysis of a DI should result in determining and predicting the clinical relevance level based on the gravity and probability of occurrence of the DI. The probability of the DI is set to 3 categories: defined, probable, and possible, whereas the gravity of the DI is grouped into 3 categories: grave, moderate, and mild. Based on the possible combinations of gravity and probability of occurrence, interactions can be grouped into 4 categories:

- Level 1 (Very high risk) resulting from the combination of: defined and grave or probable and grave. The simultaneous use of drugs is considered absolutely contraindicated.

- Level 2 (High Risk) resulting from the combinations of: possible and severe, defined and moderate, or probable and moderate. The simultaneous use of drugs is considered contraindicated: combined administration should be avoided or, if it is need, the dosage regimen of affected drug may be adjusted and to assess signs and symptoms associated to treatment effectiveness and safety, ideally in a quantitative form.

- Level 3 (Medium risk) resulting from the combination of: possible and moderate, defined and mild, or probable and mild. The simultaneous use of drugs requires assessing signs and symptoms associated to treatment effectiveness and safety, ideally in a quantitative form.

- Level 4 (Low risk) resulting from the combination can be mild. The interaction is of little clinical relevance.

From a clinical perspective, predicting of the clinical relevance of a DI should be improvement by clinical experience and knowledge obtained from situations similar; thus, the clinical interpretation of the information, including drug history and the patient's clinical condition is critical. Therefore, the presence of concomitant diseases and the need to use other drugs, the condition of renal and hepatic function, as the age and nutritional condition are factors that influence the clinical relevance of a determine DI.

\section{Clinical relevance of pharmacokinetic drug interactions in HIV-infected patients receiving antiretroviral therapy}

In this apart, using the previously described proposal, the evaluating and predicting the clinical relevance of pharmacokinetic DIs in HIV-infected patients receiving antiretroviral therapy is presented. (Amariles et al., 2007b; Giraldo et al., 2010)

\subsection{Drug interactions due to enzyme inhibition mediated by PIs or NNRTIs}

The process of evaluating and predicting the effects of this kind of DI needs to identify the drugs that are strong or moderate inhibitors of activity of different CYP450 isozymes known also as "enzyme inhibitors for excellence".

In general, PIs (ritonavir $>$ indinavir $\approx$ nelfinavir $\approx$ lopinavir $\approx$ atazanavir $\approx$ amprenavir fosamprenavir- $\approx$ darunavir $\approx$ tipranavir $>$ saquinavir) (Boffito et al., 2006), delavirdine (Tran et al., 2001) and, in some cases, efavirenz, (DeSilva et al., 2001) can inhibit the systemic metabolism 


\begin{tabular}{|c|c|c|}
\hline $\begin{array}{l}\text { Drug group or drugs } \\
\text { affected }\end{array}$ & $\begin{array}{c}\text { Clinical } \\
\text { relevance: level }\end{array}$ & Comments and suggestions \\
\hline $\begin{array}{l}\text { Antiarrhythmic drugs a } \\
\text { - Flecainide } \\
\text { - } \text { Disopyramide } \\
\text { - Amiodarone }\end{array}$ & 2: high risk & $\begin{array}{l}\text { Increased likelihood of security problems, } \\
\text { especially gastrointestinal, muscular and } \\
\text { cardiac conduction problems. } \\
\text { Recommendation: dose adjustment and } \\
\text { monitoring plasma levels }\end{array}$ \\
\hline $\begin{array}{l}\text { Antihistamines anti-H1 }{ }^{a} \\
\text { - } \quad \text { Terfenadine } \\
\text { - } \quad \text { Astemizole }\end{array}$ & $\begin{array}{l}\text { 2: high risk } \\
\text { 1: very high risk }\end{array}$ & $\begin{array}{l}\text { More likelihood of increasing QTc interval on } \\
\text { the electrocardiogram and occurrence of } \\
\text { cardiac arrhythmias, as well as dizziness } \\
\text { Recommendation: avoid co-administration }\end{array}$ \\
\hline $\begin{array}{l}\text { Ergot alkaloids } a \\
\text { - } \quad \text { Ergotamine } \\
\text { - } \text { Dihydroergotamine } \\
\text { - } \text { Ergonovine } \\
\text { - } \text { Methylergonovine } \\
\end{array}$ & 2: high risk & $\begin{array}{l}\text { Increased likelihood of ergotism: } \\
\text { hypertension, nervousness, hallucinations, } \\
\text { seizures, gastrointestinal, and muscle } \\
\text { disorders. Recommendation: dose adjustment } \\
\text { and monitoring }\end{array}$ \\
\hline $\begin{array}{l}\text { Benzodiazepines }{ }^{a} \\
\text { - } \quad \text { Midazolam } \\
\text { - } \quad \text { Alpriazolam } \\
\text { - }\end{array}$ & 2: high risk & $\begin{array}{l}\text { Increased likelihood of respiratory depression, } \\
\text { sedation and muscle weakness. Oxazepam, } \\
\text { lorazepam or temazepam are an alternative, } \\
\text { because they are eliminated by conjugation } \\
\text { with glucuronic acid and are hardly affected by } \\
\text { the simultaneous use of PIs. Recommendation: } \\
\text { dose adjustment and monitoring }\end{array}$ \\
\hline $\begin{array}{l}\text { Statins a (Aberg et al., } \\
\text { 2006; Bays, 2006; Benesic } \\
\text { et al., 2004; Cooper et al., } \\
\text { 2003; Fichtenbaum et al., } \\
\text { 2002; Fichtenbaum \& } \\
\text { Gerber, 2002; Hare et al., } \\
\text { 2002; Jacobson, 2004; Sax, } \\
\text { 2006; Sudano et al., 2006) } \\
\text { - Lovastatin } \\
\text { - Simvastatin } \\
\text { - Atorvastatin }\end{array}$ & 2: high risk & \multirow[t]{2}{*}{$\begin{array}{l}\text { Increased risk of myopathy, rhabdomyolysis, } \\
\text { and even death. Avoid the use of lovastatin or } \\
\text { simvastatin in patients using ritonavir, } \\
\text { atazanavir and saquinavir. Recommendation: } \\
\text { to use the lowest possible dose (for } \\
\text { atorvastatin) and to monitor signs and } \\
\text { symptoms of muscle toxicity, or use statins } \\
\text { involving lower risk for this type of } \\
\text { interaction, such as pravastatin, fluvastatin, or } \\
\text { rosuvastatin }\end{array}$} \\
\hline $\begin{array}{ll}\text { - } & \text { Rosuvastatin } \\
\text { - } & \text { Pluvastatin } \\
& \text { Pravastatin } \\
\end{array}$ & 3: medium risk & \\
\hline $\begin{array}{l}\text { Calcium antagonists no- } \\
\text { dihydro-pyridines }{ }^{a} \\
\text { - Verapamil } \\
\text { - Diltiazem }\end{array}$ & 2: high risk & $\begin{array}{l}\text { Increased risk of hypotension and reduced } \\
\text { cardiac conduction. It is recommended to } \\
\text { reduce the dose of these two drugs by half }\end{array}$ \\
\hline $\begin{array}{l}\text { Phosphodiesterase type } V^{a} \\
\text { - Sildenafil } \\
\text { - Tadalafil } \\
\text { - Vardenafil }\end{array}$ & 2: high risk & $\begin{array}{l}\text { Increased risk of hypotension, priapism, } \\
\text { headache, and visual disturbances. Doses } \\
\text { should be adjusted: sildenafil to } 25 \mathrm{mg} / 48 \\
\text { hours, tadalafil to } 10 \mathrm{mg} / 72 \text { hours, and } \\
\text { vardenafil to } 2.5 \mathrm{mg} / 72 \text { hours }\end{array}$ \\
\hline Cisapride $^{\mathbf{a}}$ & 2: high risk & $\begin{array}{l}\text { Increased likelihood of increasing the QTc } \\
\text { interval on the electrocardiogram and cardiac }\end{array}$ \\
\hline
\end{tabular}




\begin{tabular}{|c|c|c|}
\hline & & $\begin{array}{l}\text { arrhythmias, as well as gastrointestinal } \\
\text { disturbances and dizziness. Recommendation: } \\
\text { Dose adjustment and monitoring }\end{array}$ \\
\hline Pimozide a & 2: high risk & $\begin{array}{l}\text { Increased likelihood of involuntary } \\
\text { movements (tics), agitation, confusion, } \\
\text { behavioral disturbances, and tachycardia. } \\
\text { Recommendation: Dose adjustment and } \\
\text { monitoring }\end{array}$ \\
\hline $\begin{array}{l}\text { Warfarina } \\
\text { (acenocumarol) }\end{array}$ & 2: high risk & $\begin{array}{l}\text { The risk of bleeding may increase, when this } \\
\text { combination is used. Recommendation: dose } \\
\text { adjustment and monitoring international } \\
\text { normalized ratio (INR) }\end{array}$ \\
\hline $\begin{array}{l}\text { Fluticasone a (Arrington- } \\
\text { Sanders et al., 2006) }\end{array}$ & 2: high risk & $\begin{array}{l}\text { PIs, especially ritonavir alone or with other } \\
\text { PIs, tipranavir, and indinavir may increase } \\
\text { fluticasone levels, and even lead to the } \\
\text { development of Cushing syndrome, especially } \\
\text { with the use of ritonavir in children. } \\
\text { Recommendation: Dose adjustment and } \\
\text { monitoring }\end{array}$ \\
\hline \begin{tabular}{|l|} 
Opioid analgesics \\
(Armstrong \& Cozza, \\
2003a, 2003b) Oxycodone \\
- Oxycodone \\
- $\quad$ Buprenorphine
\end{tabular} & 2: high risk & \multirow{3}{*}{$\begin{array}{l}\text { Protease inhibitors may inhibit CYP2D6 and } \\
\text { CYP3A4 and thus the metabolism of } \\
\text { oxycodone and buprenorphine, increasing } \\
\text { their plasma concentrations as well as the } \\
\text { likelihood of toxic effects (sedation and } \\
\text { respiratory depression) } \\
\text { Dihydrocodeine, hydrocodone, and codeine are } \\
\text { pro-drugs and require activation by CYP2D6 or } \\
\text { glucuronyltransferase. Therefore, protease } \\
\text { inhibitors can inhibit the metabolism and the } \\
\text { formation of the active compound and, } \\
\text { therefore, the pharmacological effect of these } \\
\text { drugs. However, codeine is considered a } \\
\text { suitable option for pain control in HIV-infected } \\
\text { patients receiving antiretroviral therapy }\end{array}$} \\
\hline 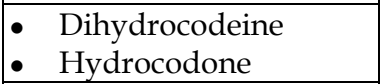 & 2: high risk & \\
\hline - Codeine & 3: medium risk & \\
\hline 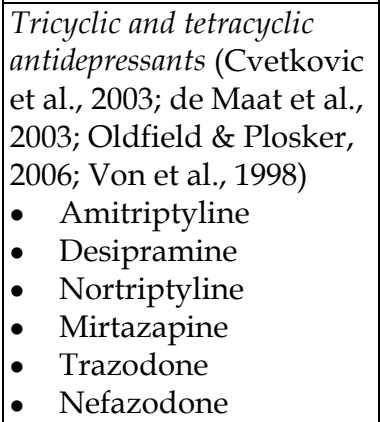 & 2: high risk & $\begin{array}{l}\text { Ritonavir alone or with another PI can inhibit } \\
\text { CYP2D6, and thus, the metabolism of these } \\
\text { drugs, which can generate toxicity problems, } \\
\text { particularly decreased conduction and cardiac } \\
\text { arrest, as well as increased anticholinergic } \\
\text { effects (constipation, dry mouth, urinary } \\
\text { retention) and cardiac abnormalities. It is } \\
\text { recommended to reduce the dose by half (or } \\
\text { use the lowest dose possible) of these two } \\
\text { drugs, with adjustments based on their } \\
\text { effectiveness and safety }\end{array}$ \\
\hline \begin{tabular}{|l|} 
Antidepressants reuptake \\
inhibitors (SSRI) (Aberg,
\end{tabular} & 2: high risk & $\begin{array}{l}\text { Ritonavir alone or with another PI can inhibit } \\
\text { CYP2D6, and thus, the metabolism of these }\end{array}$ \\
\hline
\end{tabular}




\begin{tabular}{|c|c|c|c|c|c|}
\hline \multicolumn{2}{|c|}{ 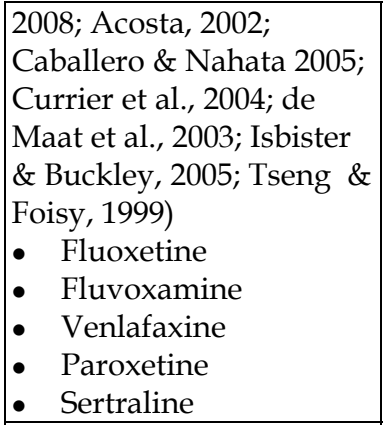 } & & & \multicolumn{2}{|c|}{$\begin{array}{l}\text { drugs, which can lead to increased levels and } \\
\text { toxicity of SSRIs (serotonin syndrome). It is } \\
\text { recommended using half or the lowest dose } \\
\text { possible of either drug, setting the dose in } \\
\text { terms of their effectiveness and safety. } \\
\text { Citalopram and possibly escitalopram and } \\
\text { sertraline, due to their low metabolism and } \\
\text { effect on CYP activity, are considered the most } \\
\text { suitable option in HIV-infected patients } \\
\text { receiving antiretroviral therapy }\end{array}$} \\
\hline \multicolumn{2}{|c|}{$\begin{array}{l}\text { Integrase inhibitors } \\
\text { Maraviroc (Aberg, 2008) }\end{array}$} & \multicolumn{2}{|c|}{ 2: high risk } & \multicolumn{2}{|c|}{$\begin{array}{l}\text { PIs increase plasma levels of maraviroc. } \\
\text { Recommendation: reduce the dose of } \\
\text { maraviroc up to } 50 \% \text { and monitoring }\end{array}$} \\
\hline \multicolumn{6}{|l|}{ With specific PIs } \\
\hline $\begin{array}{l}\text { Drug group or } \\
\text { drugs affected }\end{array}$ & \multicolumn{4}{|c|}{$\begin{array}{c}\text { Clinical } \\
\text { relevance: } \\
\text { level }\end{array}$} & Comments and suggestions \\
\hline $\begin{array}{l}\text { Simvastatin } \\
\text { (Schmidt et al., } \\
2007)\end{array}$ & \multicolumn{2}{|c|}{ Atazanavir } & \multicolumn{2}{|c|}{ 2: high risk } & $\begin{array}{l}\text { Inhibition of CYP3A4 by ATV, the } \\
\text { increase in the levels and toxicity } \\
\text { simvastatin increases the risk of } \\
\text { rhabdomyolysis and acute renal } \\
\text { failure. Recommendation: dose } \\
\text { adjustment and monitoring } \\
\end{array}$ \\
\hline \begin{tabular}{|l} 
- Efavirenz \\
- Paclitaxel \\
- Losartan \\
- Diclofenac \\
- Phenytoin \\
- Amitriptyline \\
- Omeprazole \\
- Fluoxetine \\
- Warfarin \\
- Iibuprofen \\
- Glibenclamide \\
(Dixit et al, \\
2007) \\
\end{tabular} & \multicolumn{2}{|c|}{$\begin{array}{l}\text { Ritonavir, } \\
\text { nelfinavir }\end{array}$} & \multicolumn{2}{|c|}{$\begin{array}{l}\text { 3: medium } \\
\text { risk }\end{array}$} & $\begin{array}{l}\text { Increased metabolism of drugs } \\
\text { metabolized by CYP2B6, CYP2C8, } \\
\text { CYP2C9, CYP2C19. Recommendation: } \\
\text { monitoring }\end{array}$ \\
\hline $\begin{array}{l}\text { Tenofovir (Tong } \\
\text { et al., 2007) }\end{array}$ & \multicolumn{2}{|c|}{$\begin{array}{l}A T V, P V, D R V, \\
S Q V, F P V\end{array}$} & \multicolumn{2}{|c|}{ 2: high risk } & $\begin{array}{l}\text { The coadministration of TDF with } \\
\text { ATV, LPV, DRV and SQV increased } \\
\text { Cp of tenofovir, while decreases Cp of } \\
\text { FPV. Recommendation: dose } \\
\text { adjustment and monitoring }\end{array}$ \\
\hline $\begin{array}{l}\text { Warfarin (Hughes } \\
\text { et al., 2007) }\end{array}$ & \multicolumn{2}{|l|}{$L P V / r$} & \multicolumn{2}{|c|}{$\begin{array}{l}\text { 3: medium } \\
\text { risk }\end{array}$} & $\begin{array}{l}\mathrm{LPV} / \mathrm{r} \text { may increase the metabolism of } \\
\mathrm{S} \text { enantiomer of warfarin by } \\
\text { stimulation of CYP2C9, as well as the } \\
\mathrm{R} \text { enantiomer by stimulation of }\end{array}$ \\
\hline
\end{tabular}




\begin{tabular}{|c|c|c|c|}
\hline & & & $\begin{array}{l}\text { CYP1A2. Recommendation: INR } \\
\text { monitoring }\end{array}$ \\
\hline $\begin{array}{l}\text { Minociline } \\
\text { (DiCenzo et al., } \\
\text { 2008) }\end{array}$ & $A T V$ & $\begin{array}{l}\text { 3: medium } \\
\text { risk }\end{array}$ & $\begin{array}{l}\text { Decreased levels and effect of ATV, } \\
\text { possibly due to enterohepatic cycle } \\
\text { interference associated to alterations of } \\
\text { the intestinal bacterial flora. } \\
\text { Recommendation: monitoring }\end{array}$ \\
\hline \multirow[t]{6}{*}{$\begin{array}{l}\text { Etravirine (Aberg, } \\
\text { 2008) }\end{array}$} & $A T V / r$ & $\begin{array}{l}\text { 1: very high } \\
\text { risk }\end{array}$ & $\begin{array}{l}\text { ATV Cp is decreased by } 38 \% \text {. Avoid } \\
\text { co-administration. }\end{array}$ \\
\hline & Tipranavir & $\begin{array}{l}\text { 1: very high } \\
\text { risk }\end{array}$ & $\begin{array}{l}\text { Etravirine } \mathrm{Cp} \text { was reduced by } 75 \% \text {. } \\
\text { Avoid co-administration }\end{array}$ \\
\hline & $F P V / r$ & $\begin{array}{l}\text { 1: very high } \\
\text { risk }\end{array}$ & $\begin{array}{l}\text { FPV Cp is decreased by } 77 \% . \text { Avoid } \\
\text { co-administration }\end{array}$ \\
\hline & $L P V / r$ & 2: high risk & $\begin{array}{l}\text { Etravirine Cp may be increased by } \\
85 \% . \text { Recommendation: dose } \\
\text { adjustment and monitoring }\end{array}$ \\
\hline & $D R V / r$ & $\begin{array}{l}\text { 3: medium } \\
\text { risk }\end{array}$ & $\begin{array}{l}\text { Etravirine Cp may be decreased by } \\
50 \% \text {. Recommendation: monitoring }\end{array}$ \\
\hline & $N F V$ & 2: high risk & $\begin{array}{l}\text { Concomitant use increase plasma } \\
\text { concentrations of nelfinavir, due to the } \\
\text { inhibitory effect of etravirine on } \\
\text { CYP3A4. Recommendation: dose } \\
\text { adjustment and monitoring (Schöller } \\
\text { et al., 2006a; Sekar et al., 2006a) }\end{array}$ \\
\hline \multirow[t]{2}{*}{$\begin{array}{l}\text { Raltegravir } \\
(\text { Aberg, 2008) }\end{array}$} & Tipranavir & 2: high risk & $\begin{array}{l}\text { Plasma concentrations of raltegravir } \\
\text { are reduced when it is used with } \\
\text { tipranavir. Recommendation: dose } \\
\text { adjustment and monitoring }\end{array}$ \\
\hline & $A T V$ & 2: high risk & $\begin{array}{l}\text { Plasma concentrations of raltegravir } \\
\text { are increased when it is used with } \\
\text { ATV. Recommendation: dose } \\
\text { adjustment and monitoring }\end{array}$ \\
\hline \multirow[t]{4}{*}{ Darunavir } & \begin{tabular}{|l|}
$S Q V($ Sekar et \\
al., 2006a) \\
\end{tabular} & 2: high risk & \multirow{4}{*}{$\begin{array}{l}\text { Plasma concentrations and effects of } \\
\text { these PIs may be increased, due to the } \\
\text { inhibitory effect of darunavir. } \\
\text { Recommendation: dose adjustment and } \\
\text { monitoring }\end{array}$} \\
\hline & \begin{tabular}{|l|}
$L P V$ (Sekar et \\
al., 2006b) \\
\end{tabular} & 2: high risk & \\
\hline & $I D V$ & $\begin{array}{l}\text { 3: medium } \\
\text { risk }\end{array}$ & \\
\hline & $N V P$ & $\begin{array}{l}\text { 3: medium } \\
\text { risk }\end{array}$ & \\
\hline $\begin{array}{l}\text { - Pravastatin } \\
\text { - Sildenafil } \\
\text { - Vardenafil } \\
\text { - Tadalafil }\end{array}$ & $\begin{array}{l}D R V \text { (Sekar et } \\
\text { al., 2008) }\end{array}$ & 2: high risk & $\begin{array}{l}\text { Plasma concentrations and effects of } \\
\text { these medications may be increased, } \\
\text { due to the inhibitory effect of } \\
\text { darunavir. Recommendation: dose } \\
\text { adjustment and/or monitoring } \\
\end{array}$ \\
\hline $\begin{array}{l}\text { - Budesonide } \\
\text { - Quinine }\end{array}$ & $\begin{array}{l}R T V \text { and } L P V / r \\
\text { (Daveluy et al, }\end{array}$ & 2: high risk & $\begin{array}{l}\text { Plasma concentrations and effects of } \\
\text { these medications may be increased, }\end{array}$ \\
\hline
\end{tabular}




\begin{tabular}{|c|c|c|c|}
\hline $\begin{array}{l}\text { - Docetaxel } \\
\text { - Fluticasone } \\
\text { - Oxycodone } \\
\text { - Alprazolam } \\
\text { - Sirolimus } \\
\text { - Quetiapine }\end{array}$ & $\begin{array}{l}\text { 2009; Gray et } \\
\text { al., 2010; } \\
\text { Gruber \& } \\
\text { McCance-Katz, } \\
\text { 2010) }\end{array}$ & & $\begin{array}{l}\text { due to the inhibitory effect of } \\
\text { ritonavir. Recommendation: dose } \\
\text { adjustment and monitoring }\end{array}$ \\
\hline $\begin{array}{l}\text { Cannabinoids } \\
\text { (Abrams et al., } \\
2003 \text { ) }\end{array}$ & $\begin{array}{l}\text { IDV } \\
\text { NFV } \\
\text { RTV }\end{array}$ & 2: high risk & $\begin{array}{l}\text { Plasma concentrations and effects of } \\
\text { cannabinoids and marihuana derivates } \\
\text { may be increased, due to the } \\
\text { inhibitory effect of PIs. } \\
\text { Recommendation: dose adjustment } \\
\text { and monitoring, or avoid use of } \\
\text { cannabinoids }\end{array}$ \\
\hline \multirow[t]{5}{*}{ Methadone } & $\begin{array}{l}L P V / r \\
\text { (McCance-Katz } \\
\text { et al., 2003) }\end{array}$ & 2: high risk & \multirow{5}{*}{$\begin{array}{l}\text { Plasma concentrations and effects of } \\
\text { methadone may be increased, due to } \\
\text { inhibitory effect of PIs on CYP3A4. } \\
\text { Recommendation: dose adjustment of } \\
\text { methadone and/or monitoring } \\
\text { adverse effects of methadone }\end{array}$} \\
\hline & $\begin{array}{l}N F V \\
\text { (McCance-Katz } \\
\text { et al., 2004) }\end{array}$ & 2: high risk & \\
\hline & $\begin{array}{l}\text { ATV (Friedland } \\
\text { et al, 2005) }\end{array}$ & $\begin{array}{l}\text { 3: medium } \\
\text { risk }\end{array}$ & \\
\hline & $\begin{array}{l}F P V \text { (Cao et al., } \\
2008) \\
\end{array}$ & $\begin{array}{l}\text { 3: medium } \\
\text { risk }\end{array}$ & \\
\hline & $I D V$ & $\begin{array}{l}\text { 3: medium } \\
\text { risk }\end{array}$ & \\
\hline $\begin{array}{l}\text { Quetiapine } \\
\text { (Hantson et al., } \\
2010)\end{array}$ & $A T V$ & 2: high risk & $\begin{array}{l}\text { Plasma concentrations and effects of } \\
\text { quetiapine are increased, due to } \\
\text { inhibitory effect of atazanavir. } \\
\text { Recommendation: dose adjustment of } \\
\text { quetiapine and monitoring }\end{array}$ \\
\hline $\begin{array}{l}\text { Antineoplasics } \\
\text { (Levêque et al., } \\
\text { 2009; Makinson et } \\
\text { al., 2010) } \\
\text { - Irinotecan } \\
\text { - Vinblastine } \\
\text { - Vincristine }\end{array}$ & $L P V / r$ & $\begin{array}{l}\text { 3: medium } \\
\text { risk }\end{array}$ & $\begin{array}{l}\text { Plasma concentrations and effects of } \\
\text { these antineoplastics may be } \\
\text { increased, due to inhibitory effect of } \\
\text { LPV/r on CYP3A5. } \\
\text { Recommendation: monitoring }\end{array}$ \\
\hline
\end{tabular}

a Protease inhibitors (ritonavir $\rightarrow$ indinavir $\approx$ nelfinavir $\approx$ atazanavir $\approx$ amprenavir- fosamprenavir $-\approx$ tipranavir $\rightarrow$ saquinavir), mainly through inhibition of CYP3A4, may decrease the metabolism of these drugs, which can cause increased plasma concentrations and toxicity.

Table 2. General interactions due to enzyme inhibition by protease inhibitors (Boffito et al., 2006; Busti et al., 2004; DeSilva et al., 2001; DHHS, 2011; Krikorian \& Rudorf, 2005; Piscitelli \& Gallicano, 2001; Kashuba, 2005a; Robertson et al., 2005a; Tran et al., 2001; Winston \& Boffito, 2005; Wire et al., 2006)

of several drugs, increase their plasma levels, and may cause adverse drug reactions, which could cause grave health problems in patients. Thus, according to their clinical relevance, most 
could be classified in level 1 or 2 . Table 2 lists the interactions mediated by enzyme inhibition caused by PIs. Table 3 contains the interactions mediated by delavirdine and efavirenz.

\begin{tabular}{|c|c|c|c|}
\hline \multirow{2}{*}{$\begin{array}{l}\text { Drug group or drugs } \\
\text { affected }\end{array}$} & \multicolumn{2}{|c|}{ Clinical relevance: level } & \multirow[t]{2}{*}{ Comments and suggestions } \\
\hline & Delavirdine & Efavirenz & \\
\hline $\begin{array}{l}\text { Anti-H1 antihistamines }{ }^{a} \\
\text { - Terfenadine }\end{array}$ & 2: high risk & 2: high risk & \multirow{2}{*}{$\begin{array}{l}\text { More likely to increase QTc interval } \\
\text { on the electrocardiogram and } \\
\text { cardiac arrhythmias, as well as } \\
\text { dizziness. Recommendation: avoid } \\
\text { co-administration }\end{array}$} \\
\hline - Astemizole & $\begin{array}{l}\text { 1: very high } \\
\text { risk }\end{array}$ & $\begin{array}{l}\text { 1: very high } \\
\text { risk }\end{array}$ & \\
\hline $\begin{array}{l}\text { Ergot alkaloids }{ }^{a} \\
\text { - Ergotamine } \\
\text { - Dihydroergotamine } \\
\text { - Ergonovine } \\
\text { - Methylergonovine }\end{array}$ & 2: high risk & 2: high risk & $\begin{array}{l}\text { Increased likelihood of ergotism: } \\
\text { hypertension, nervousness, } \\
\text { hallucinations, seizures, } \\
\text { gastrointestinal and muscle } \\
\text { disorders. Recommendation: dose } \\
\text { adjustment and monitoring }\end{array}$ \\
\hline $\begin{array}{l}\text { Benzodiazepines }^{a} \\
\text { - } \quad \text { Midazolam } \\
\text { - } \quad \text { Triazolam } \\
\text { - } \quad \text { Alprazolam }\end{array}$ & 2: high risk & 2: high risk & $\begin{array}{l}\text { Increased likelihood of respiratory } \\
\text { depression, sedation and muscle } \\
\text { weakness. Oxazepam, lorazepam } \\
\text { or temazepam are an alternative, } \\
\text { because they are eliminated by } \\
\text { conjugation with glucuronic acid } \\
\text { and are hardly affected by the } \\
\text { simultaneous use of PI }\end{array}$ \\
\hline $\begin{array}{l}\text { Statins (Bays, 2006; } \\
\text { Cooper et al., 2003; } \\
\text { Fichtenbaum \& Gerber, } \\
\text { 2002; Jacobson, 2004; Sax, } \\
\text { 2006; Sudano et al., 2006.) } \\
\text { - Lovastatin } \\
\text { - Simvastatin } \\
\text { - } \quad \text { Atorvastatin }\end{array}$ & 2: high risk & \multirow[t]{2}{*}{$\begin{array}{l}\text { Efavirenz } \\
\text { acts as an } \\
\text { inducer of } \\
\text { statins } \\
\text { metabolism } \\
\text { (see } \\
\text { interactions } \\
\text { induction) }\end{array}$} & \multirow[t]{2}{*}{$\begin{array}{l}\text { Increased risk of myopathy, } \\
\text { rhabdomyolysis, and even death. It } \\
\text { should be avoided in patients using } \\
\text { delavirdine (see text: 3.1.1 statins } \\
\text { with PIs or delavirdine) }\end{array}$} \\
\hline $\begin{array}{ll}\text { - } & \text { Rosuvastatin } \\
\text { - } & \text { Fluvastatin } \\
\text { - Pravastatin }\end{array}$ & $\begin{array}{l}\text { 3: medium } \\
\text { risk }\end{array}$ & & \\
\hline $\begin{array}{l}\text { Calcium antagonists not } \\
\text { dihydro-pyridine }{ }^{a} \\
\text { - Verapamil }\end{array}$ & 2: high risk & \multirow[t]{2}{*}{$\begin{array}{l}\text { Information } \\
\text { not } \\
\text { available }\end{array}$} & \multirow{2}{*}{$\begin{array}{l}\text { Especially with delavirdine, it } \\
\text { increases the risk of hypotension } \\
\text { and reduced cardiac conduction. } \\
\text { Consider to use the half dosing } \\
\text { schedule of these two drugs }\end{array}$} \\
\hline - Diltiazem & $\begin{array}{l}\text { 3: medium } \\
\text { risk }\end{array}$ & & \\
\hline $\begin{array}{l}\text { Phosphodiesterase Type V } \\
\text { inhibitors a } \\
\text { - Sildenafil } \\
\text { - Tadalafil } \\
\text { - Vardenafil }\end{array}$ & 2: high risk & 2: high risk & $\begin{array}{l}\text { It increases the risk of hypotension, } \\
\text { priapism, headache, and visual } \\
\text { disturbances. Doses should be } \\
\text { adjusted: sildenafil to } 25 \mathrm{mg} / 48 \\
\text { hours, tadalafil to } 10 \mathrm{mg} / 72 \text { hours, } \\
\text { and vardenafil to } 2.5 \mathrm{mg} / 72 \text { hours }\end{array}$ \\
\hline
\end{tabular}




\begin{tabular}{|c|c|c|c|}
\hline $\begin{array}{l}\text { Opioid analgesics } \\
\text { (Armstrong \& Cozza, } \\
\text { 2003a, 2003b) } \\
\text { - Oxycodone } \\
\text { - Buprenorphine }\end{array}$ & 2: high risk & \begin{tabular}{|l|} 
No \\
information \\
available on \\
clinically \\
relevant \\
interactions
\end{tabular} & $\begin{array}{l}\text { Inhibition of CYP3A4 and } \\
\text { metabolism of oxycodone and } \\
\text { buprenorphine, increasing plasma } \\
\text { concentrations and the likelihood } \\
\text { of toxic effects (sedation and } \\
\text { respiratory depression) }\end{array}$ \\
\hline $\begin{array}{l}\text { - } \text { Dihydrocodeine } \\
\text { - Hydrocodone } \\
\text { - } \text { Codeine }\end{array}$ & $\begin{array}{l}\text { 3: medium } \\
\text { risk }\end{array}$ & & $\begin{array}{l}\text { Dihydrocodeine, hydrocodone, and } \\
\text { codeine are pro-drugs and require } \\
\text { activation by CYP2D6 or } \\
\text { glucuronyl-transferase. Therefore, } \\
\text { delavirdine may inhibit the } \\
\text { metabolism and the formation of } \\
\text { the active compound and, } \\
\text { consequently, the pharmacological } \\
\text { effect of these drugs }\end{array}$ \\
\hline $\begin{array}{l}\text { Antidepressants reuptake } \\
\text { inhibitors (SSRI) } \\
\text { (Caballero \& Nahata, } \\
\text { 2005; Currier et al., 2004; } \\
\text { DeSilva et al., 2001; } \\
\text { Isbister \& Buckley, 2005; } \\
\text { Tseng \& Foisy, 1999) } \\
\text { - Fluoxetine } \\
\text { - Fluvoxamine } \\
\text { - Venlafaxine } \\
\text { - Paroxetine } \\
\text { - Sertraline }\end{array}$ & $\begin{array}{l}\text { No } \\
\text { information } \\
\text { available on } \\
\text { clinically } \\
\text { relevant } \\
\text { interactions } \\
\text { with } \\
\text { delavirdine }\end{array}$ & 2: high risk & $\begin{array}{l}\text { Efavirenz, in cases of deficiency of } \\
\text { CYP2D6, may inhibit CYP2C9 and } \\
\text { CYP2C19, which can lead to } \\
\text { increased levels and toxicity of } \\
\text { SSRIs (serotonin syndrome). We } \\
\text { recommend using half or the } \\
\text { lowest dose possible of these two } \\
\text { drugs, adjusting the dose in terms } \\
\text { of their effectiveness and safety }\end{array}$ \\
\hline Warfarin ${ }^{a}$ (acenocumarol) & 2: high risk & \begin{tabular}{|l|} 
Efavirenz \\
induces \\
warfarin \\
metabolism \\
\end{tabular} & $\begin{array}{l}\text { With delavirdine it increases the } \\
\text { risk of bleeding. It is recommended } \\
\text { dose adjustment and monitoring of } \\
\text { INR }\end{array}$ \\
\hline Cisapride $^{a}$ & 2: high risk & 2: high risk & $\begin{array}{l}\text { More likelihood of increasing QTc } \\
\text { interval on the electrocardiogram } \\
\text { and cardiac arrhythmias, as well as } \\
\text { gastrointestinal disturbances and } \\
\text { dizziness }\end{array}$ \\
\hline Raltegravir (Aberg, 2008) & $\begin{array}{l}\text { No } \\
\text { information } \\
\text { available }\end{array}$ & 2: high risk & $\begin{array}{l}\text { The Cp of raltegravir is reduced } \\
\text { when is co-administered with } \\
\text { efavirenz }\end{array}$ \\
\hline
\end{tabular}

a Delavirdine, primarily through inhibition of CYP3A4, and efavirenz through inhibition of CYP3A4 and CYP2D6, may decrease the metabolism of these drugs, which can cause increased plasma concentrations and toxicity.

Table 3. General interactions due to enzyme inhibition by inhibitors of non-nucleoside transcriptase (DeMaat et al., 2003; DHHS, 2011; Krikorian \& Rudorf, 2005; Piscitelli \& Gallicano, 2001; Tran et al., 2001) 
From a practical perspective and specifying the type of PI, it is generally recommended to avoid the following combinations (level 1 or level 2: very high risk or high risk): (DHHS, 2011)

- Indinavir with: atazanavir, simvastatin or lovastatin, amiodarone, cisapride, pimozide, astemizole or terfenadine, midazolam or triazolam, ergotamine, ergonovine, or methyl ergonovine.

- Ritonavir (alone or with another PI) with: voriconazole (with a ritonavir dose higher than $400 \mathrm{mg} / 12$ hours), fluticasone, simvastatin or lovastatin, amiodarone, flecainide, propafenone, or quinidine, cisapride, pimozide or clozapine, trazodone or nefazodone, astemizole or terfenadine, midazolam or triazolam, ergotamine, ergonovine or methylergonovine.

- Saquinavir with: simvastatin or lovastatin, cisapride, pimozide, astemizole or terfenadine, midazolam or triazolam, ergotamine, ergonovine, or methyl ergonovine.

- Lopinavir/ritonavir with: fluticasone, simvastatin or lovastatin, flecainide or propafenone, cisapride, pimozide, astemizole or terfenadine, midazolam or triazolam, ergotamine, ergonovine, or methyl ergonovine.

- Nelfinavir with indinavir, irinotecan, simvastatin or lovastatin, cisapride, pimozide, astemizole or terfenadine, midazolam or triazolam, ergotamine, ergonovine or methylergonovine.

- Atazanavir with: simvastatin or lovastatin, cisapride, pimozide, astemizole or terfenadine, midazolam or triazolam, ergotamine, ergonovine, or methyl ergonovine.

- Amprenavir (same for the fosamprenavir) with: simvastatin or lovastatin, cisapride, pimozide, astemizole or terfenadine, midazolam or triazolam, ergotamine, ergonovine, or methyl-ergonovina. ( Wire et al., 2006)

- Tipranavir with: fluticasone, simvastatin or lovastatin, amiodarone, flecainide, propafenone, or quinidine, cisapride, pimozide, astemizole or terfenadine, midazolam or triazolam, ergotamine, ergonovine, or methyl-ergonovina.( Boffito et al., 2006)

From a practical perspective and specifying the type of NNRTIs, it is generally recommended to avoid the following combinations (level 1 or level 2: very high risk or high risk): (DHHS, 2011)

- Delavirdine with: amprenavir or fosamprenavir, simvastatin or lovastatin, cisapride, astemizole or terfenadine, alprazolam, midazolam or triazolam, ergotamine, ergonovine or methyl ergonovine.

- Efavirenz with: voriconazole, cisapride, astemizole or terfenadine, midazolam or triazolam, ergotamine, ergonovine or methyl ergonovine.

Related to PIs and NNRTIs Di, it is important to note that for delavirdine, its combination with amprenavir or fosamprenavir can cause a decrease in the levels and effects of delavirdine. (Tran et al., 2001; Wire et al., 2006) Additionally, some studies have shown a possible interaction between fosamprenavir with lopinavir/ritonavir, in fact mediated by a two-way increase in CYP3A4 and/or P-glycoprotein (P-gp) metabolic activity by fosamprenavir and lopinavir. (Huang et al., 2001; Taburet et al., 2004; Tranet al., 2002) This interaction is associated to a $50-60 \%$ decrease in lopinavir plasma concentration, and a $60-$ $70 \%$ decrease in amprenavir levels (ritonavir levels do not change significantly). Consequently, combining these 3 PIs may be inappropriate, due to a high probability of generating a virologic failure (Kashuba et al., 2005) (level 2: high risk). The negative effect of this interaction is not neutralized by increasing ritonavir dose from 100 to $200 \mathrm{mg} / 12$ hours; while this strategy is associated with increased gastric problems associated to the use of ritonavir. (Mauss et al., 2004) 
On the other hand, it seems that the combination atazanavir with lopinavir/ritonavir does not generate this problem (although decreased levels of lopinavir by the action of atazanavir may occur (Colombo et al., 2006), which is considered a double pharmacokinetic extension (boosted) with PI, useful in a certain group of patients. (Ribera et al., 2006) Contrary to this effect (of atazanavir on lopinavir levels), when using a saquinavir/ritonavir and atazanavir $(1.600 / 100$ and $200 \mathrm{mg})$ an increase in saquinavir plasma and cellular levels is observed (without effect on ritonavir). Therefore, it is believed that adding atazanavir $200 \mathrm{mg} /$ day to saquinavir/ritonavir $1.600 / 100 \mathrm{mg} /$ day may be a good strategy for patients in who the Cpss are under the minimum effective one. (Ford et al., 2006)

Due to it is common used of this group of drugs in HIV infected patients receiving antiretroviral therapy; it is important focus following drugs therapeutic class:

Statins. The simultaneous use of PI or delavirdine with statins (lovastatin $\approx$ simvastatin $>$ atorvastatin $>$ rosuvastatin $\approx$ fluvastatin $\approx$ pravastatin) increases the risk of myopathy, rhabdomyolysis, and even death. (Hare et al., 2002) Thus, for atorvastatin the lowest possible doses are recommended. Also, it is suggested to monitor muscle toxicity signs and symptoms or to use statins less likely to have this type of interaction, such as pravastatin, fluvastatin or rosuvastatin from. (Benesic et al., 2004; DHHS, 2011; Fichtenbaum et al., 2002; Fichtenbaum \& Gerber, 2002; Jacobson, 2004; Sax, 2006) In this regard, statins (simvastatin, lovastatin, and atorvastatin, except pravastatin, fluvastatin, and rosuvastatin) are metabolized by CYP3A4 and, therefore, their use should be avoided in patients using PIs, especially ritonavir, atazanavir and saquinavir. Both metabolism and levels of pravastatin, as well as, most likely, fluvastatin and rosuvastatin are slightly affected by the combined use of ritonavir, indinavir, atazanavir, saquinavir, and nelfinavir and therefore they could be combined in patients receiving cART or HAART. (Aberg et al., 2006; Bays, 2006; Benesic et al., 2004; Cooper et al., 2003; Jacobson, 2004; Sudano et al., 2006). However, in some cases it may be necessary to increase the dose of pravastatin, as appears likely in the case of nelfinavir. (Aberg et al., 2006)

Selective serotonin reuptake inhibitors (SSRI). The use of ritonavir (alone or in combination with another PI) by inhibiting CYP2D6 and efavirenz by inhibiting CYP2C9 and CYP2C19, when a deficit of CYP2D6 exists, may lead to increased levels and toxic effects of SSRIs (fluoxetine, fluvoxamine, venlafaxine, paroxetine and, sertraline). Particularly, it may lead to the development of serotonin syndrome (DeSilva et al., 2001). Therefore, it is advisable to use half the dose or the lowest dose possible of these drugs, setting the dose in terms of their effectiveness and safety. (DHHS, 2011) Additionally, citalopram, and possibly escitalopram and sertraline, due to their pharmacological properties (low metabolism and minimal effect on CYP activity), are considered the most suitable option in patients receiving cART or HAART. (Caballero \& Nahata, 2005; Currier et al., 2004; DHHS, 2011; Kashuba, 2005)

Generally, serotonin syndrome is a disorder caused by an increase in serotonin levels, resulting in cognitive disorders (lethargy, confusion, coma, agitation, hallucinations, and seizures); neuromuscular activity disorders (myoclonus, tremor, hyperreflexia, rigidity, hyperactivity); autonomic nervous system disorders (hypotension or hypertension, tachycardia, chills, hyperthermia, diaphoresis, diarrhea, salivation, abdominal pain, tachypnea) (Isbister \& Buckley, 2005). In a patient under treatment with a SSRI (or other drugs with serotonergic activity in the central nervous system), such change can occur due to pharmacodynamic interactions (simultaneous treatment with other drugs that increase serotonin activity or agonist) or to pharmacokinetic interactions (treatment with an CYP2D6 
inhibitor or because of a situation leading to an increase in serotonin levels and effects). (DeSilva et al., 2001; Tseng \& Foisy, 1999)

\subsection{Drug interactions mediated mainly by enzymatic inhibition of PIs and NNRTIs with other known enzyme inhibitors (for some drugs metabolism inhibition may occur while for others may occur metabolism induction)}

Since PIs and NNRTIs are metabolized by CYP3A, strong inhibitors of CYP3A isoenzyme may inhibit its metabolism, increase plasma levels and therefore the risk of developing adverse drug reactions or toxicities. In these cases, generally, inhibition is bidirectional (the strong inhibitors may increase the PIs and NNRTIs levels and toxicity as well as PIs and NNRTIs may increase the inhibitors plasma concentrations and toxicity). However, in some cases the interaction can be in one direction, while in others both NNRTI metabolism inhibition (by the known inhibitor) and known inhibitor metabolism induction (by the NNRTIs) may occur, especially with efavirenz and nevirapine. In table 4 (for PIs) and table 5 (for NNRTIs) the most relevant interactions of this type are included.

Due to it is important some examples of the one-way drug interactions mediated mainly by enzymatic inhibition of PIs and NNRTIs are detailed:

Azoles antifungals

- Azoles antifungals (ketoconazole, itraconazole, fluconazole, voriconazole) with amprenavir. Antifungal may inhibit amprenavir hepatic metabolism (and probably fosamprenavir metabolism), which can lead to an increase in their levels, although such increase does not seem to be clinically relevant (level 3: medium risk). (Vourvahis \& Kashuba, 2007)

- Fluconazole with tipranavir/ritonavir. Fluconazole causes an increase in the area under the curve. In any case, similar to what happens when PIs are used with azoles antifungals; it is advisable to monitor the hepatic function and not to exceed a dose of $200 \mathrm{mg} /$ daily fluconazole. (DHHS, 2011)

- Ketoconazole with delavirdine. Ketoconazole may increase delavirdine levels (Level 3: medium risk), (DHHS, 2011) but ketoconazole levels do not change significantly.

Macrolides and immunosuppressants

- Clarithromycin with amprenavir (and probably fosamprenavir). Clarithromycin produces an $18 \%$ an increase in the area under the curve of amprenavir, while the effect of PI on macrolide levels is lowest. (Brophy et al., 2000)

- Immunosuppressants with efavirenz or nevirapine. Cyclosporine and tacrolimus may increase the levels and toxicity of these two NNRTIs. It is thus recommended to monitor toxicity signs and symptoms of these drugs and, if required, to adjust its dose (Level 3: medium risk). (Jain et al., 2002; Vogel et al., 2004)

3.3 Drug interactions due to enzyme induction of NNRTIS (Back et al., 2003; Bergshoeff et al., 2005; DHHS, 2011; Kashuba, 2005; Krikorian \& Rudorf, 2005; Mildvan et al., 2002; (Pérez et al., 2009; Piscitelli \& Gallicano, 2001; Saraga et al., 2006; Young, 2005)

Efavirenz and nevirapine due to their ability to increase the hepatic metabolism may cause a decrease in PIs levels and consequently therapeutic failure. Therefore, in general, using a single PI with efavirenz (in particular) or nevirapine is considered contraindicated (level 2 interaction: high risk). For example, efavirenz can cause a $39 \%$ reduction in the minimum 


\begin{tabular}{|c|c|c|}
\hline $\begin{array}{l}\text { Group drugs or } \\
\text { drugs affected }\end{array}$ & \begin{tabular}{|c|} 
Clinical \\
relevance of the \\
bidirectional \\
inhibition: level
\end{tabular} & Comments and suggestions \\
\hline $\begin{array}{l}\text { Azole antifungals a } \\
\text { (Polk et al., 1999; } \\
\text { Vourvahis \& } \\
\text { Kashuba, 2007) } \\
\text { - Ketoconazole } \\
\text { - Itraconazole } \\
\text { - Fluconazole }\end{array}$ & 2: high risk & $\begin{array}{l}\text { Except for amprenavir and fosamprenavir, there is a } \\
\text { bidirectional increase in the levels of antifungal and } \\
\text { PIs, which can lead to toxicity problems. It is } \\
\text { recommended using up to } 200 \mathrm{mg} / \text { day of } \\
\text { ketoconazole or } 200-400 \mathrm{mg} / \text { day of itraconazole. In } \\
\text { the case of fluconazole combination with tipranavir / } \\
\text { ritonavir an increase in levels of tipranavir, without } \\
\text { significant changes in fluconazole is observed. } \\
\text { (Vourvahis \& Kashuba, 2007) }\end{array}$ \\
\hline - Voriconazole & 3: medium risk & $\begin{array}{l}\text { The coadministration of darunavir and ketoconazole } \\
\text { increase the plasma concentrations of both drugs, } \\
\text { creating the same toxic effects (Sekar et al., 2008) }\end{array}$ \\
\hline $\begin{array}{l}\text { Macrolides a } \\
\text { - Erythromycin } \\
\text { - Clarithromycin }\end{array}$ & 2: high risk & $\begin{array}{l}\text { Except for amprenavir and fosamprenavir, a } \\
\text { bidirectional increase in the levels of macrolides and } \\
\text { PIs is generated, which can lead to toxicity problems. } \\
\text { Macrolides may increase QTc interval on the } \\
\text { electrocardiogram. It is recommended to use } \\
\text { maximum } 1 \mathrm{~g} / \text { day of these antibiotics (for } \\
\text { clarithromycin it is suggested to reduce } 50-75 \% \text { in the } \\
\text { level, if the patient has a creatinine clearance }<60 \mathrm{ml} \\
\text { / minute). In general, it is considered that using } \\
\text { erythromycin with a strong inhibitors of CYP3A4 } \\
\text { should be avoided (Ray et al., 2004) }\end{array}$ \\
\hline $\begin{array}{l}\text { Immuno- } \\
\text { suppressants a } \\
\text { (Jain et al., 2002; } \\
\text { Vogel et al., 2004) } \\
\text { - Cyclosporine } \\
\text { - Tacrolimus }\end{array}$ & 2: high risk & $\begin{array}{l}\text { Increased levels and toxicity of immunosuppressants } \\
\text { can be generated, so it is recommended to monitor } \\
\text { plasma levels and to adjust the treatment regime. In } \\
\text { the case of nelfinavir/tacrolimus combination it is } \\
\text { recommended to reduce by } 50 \% \text { the dose of the } \\
\text { immunosuppressant (Jain et al., 2002). In the case of } \\
\text { cyclosporine with lopinavir / ritonavir, a } 5 \% \text { initial } \\
\text { reduction is recommended in cyclosporine dose (a } \\
\text { reduction of up to } 20 \% \text { ) may be necessary. (Vogel et } \\
\text { al., 2004) Cyclosporine and tacrolimus may increase } \\
\text { the levels and toxicity of PIs and therefore it is } \\
\text { recommended to monitor signs and symptoms of } \\
\text { toxicity, and if required, to adjust the dose }\end{array}$ \\
\hline
\end{tabular}

a PIs can inhibit CYP3A4 and may decrease the metabolism of these drugs, which can cause increased plasma concentrations and toxicity. In turn, these drugs, also due to CYP3A4 inhibition, may decrease the metabolism of PIs and increase plasma concentrations and toxicity.

Table 4. Clinical relevant bidirectional drug interactions mediated by PIs enzyme inhibition with other known drugs (Brophy et al., 2000; DHHS, 2011; Kashuba, 2005a, 2005b; Krikorian \& Rudorf, 2005; Robertson et al., 2005b; Young, 2005) 


\begin{tabular}{|c|c|c|c|c|}
\hline \multirow{2}{*}{$\begin{array}{l}\text { Drug group or drugs } \\
\text { affected }\end{array}$} & \multicolumn{3}{|c|}{ Clinical relevance: level } & \multirow{2}{*}{$\begin{array}{l}\text { Comments and } \\
\text { suggestions }\end{array}$} \\
\hline & Delavirdine & Efavirenz & Nevirapine & \\
\hline $\begin{array}{l}\text { Azole antifungals a } \\
\text { - Ketoconazole }\end{array}$ & $\begin{array}{l}\text { 3: medium } \\
\text { risk }\end{array}$ & $\begin{array}{l}\text { No } \\
\text { information } \\
\text { available }\end{array}$ & $\begin{array}{l}\text { 3: medium } \\
\text { risk }\end{array}$ & $\begin{array}{l}\text { With Delavirdine: } \\
\text { Delavirdine levels } \\
\text { increased with no } \\
\text { change in ketoconazole } \\
\text { levels. } \\
\text { With nevirapine: } \\
\text { increased levels of } \\
\text { nevirapine and } \\
\text { decreased ketoconazole } \\
\text { levels }\end{array}$ \\
\hline - Itraconazole & $\begin{array}{l}\text { 3: medium } \\
\text { risk }\end{array}$ & $\begin{array}{l}\text { No } \\
\text { information } \\
\text { available }\end{array}$ & $\begin{array}{l}\text { No } \\
\text { information } \\
\text { available }\end{array}$ & $\begin{array}{l}\text { In general, slight clinical } \\
\text { relevance }\end{array}$ \\
\hline - Fluconazole & $\begin{array}{l}\text { No } \\
\text { significant } \\
\text { changes }\end{array}$ & $\begin{array}{l}\text { No } \\
\text { information } \\
\text { available }\end{array}$ & 2: high risk & $\begin{array}{l}\text { With nevirapine: } \\
\text { increased levels and } \\
\text { potential liver toxicity of } \\
\text { nevirapine and no } \\
\text { changes on fluconazole }\end{array}$ \\
\hline - Voriconazole & $\begin{array}{l}\text { 3: medium } \\
\text { risk }\end{array}$ & 2: high risk & $\begin{array}{l}\text { 3: medium } \\
\text { risk }\end{array}$ & $\begin{array}{l}\text { With delavirdine: } \\
\text { increased levels of both } \\
\text { drugs. The toxic effects } \\
\text { of both drugs should be } \\
\text { monitored. } \\
\text { Efavirenz and } \\
\text { nevirapine: NNRTI } \\
\text { levels increased and } \\
\text { decreased levels } \\
\text { voriconazole }\end{array}$ \\
\hline $\begin{array}{l}\text { Macrolides a } \\
\text { - Erythromycin } \\
\text { - Clarithromycin }\end{array}$ & 2: high risk & 2: high risk & $\begin{array}{l}\text { No } \\
\text { information } \\
\text { available }\end{array}$ & $\begin{array}{l}\text { A bidirectional increase } \\
\text { in the levels of } \\
\text { macrolides and } \\
\text { delavirdine and } \\
\text { efavirenz, which may } \\
\text { lead to problems of } \\
\text { toxicity of macrolides } \\
\text { and NNRTIs } \\
\end{array}$ \\
\hline $\begin{array}{l}\text { Immunosuppressant } \\
\text { - Cyclosporine } \\
\text { - Tacrolimus }\end{array}$ & $\begin{array}{l}\text { 3: medium } \\
\text { risk }\end{array}$ & $\begin{array}{l}\text { 3: medium } \\
\text { risk }\end{array}$ & $\begin{array}{l}\text { 3: medium } \\
\text { risk }\end{array}$ & $\begin{array}{l}\text { Immunosuppressants } \\
\text { inhibit the metabolism } \\
\text { of efavirenz and } \\
\text { nevirapine. In addition, } \\
\text { there are reports of } \\
\text { decreased levels of } \\
\text { cyclosporine by }\end{array}$ \\
\hline
\end{tabular}




\begin{tabular}{|l|l|l|l|l|}
\hline & & & & $\begin{array}{l}\text { efavirenz, so it is } \\
\text { recommended to } \\
\text { monitor the levels of } \\
\text { immunosuppressants } \\
\text { (Tseng et al., 2002) }\end{array}$ \\
\hline $\begin{array}{l}\text { Pimozide (DHHS, } \\
\text { 2011) }\end{array}$ & $\begin{array}{l}\text { No } \\
\text { information } \\
\text { available }\end{array}$ & 2: high risk & $\begin{array}{l}\text { No } \\
\text { information } \\
\text { available }\end{array}$ & $\begin{array}{l}\text { Efavirenz with drugs } \\
\text { primarily metabolized } \\
\text { by CYP3A4 may } \\
\text { increase plasma } \\
\text { concentrations of drugs, } \\
\text { due to a weak inhibitory } \\
\text { effect on this enzyme. } \\
\text { Recommendation: There } \\
\text { may be potential for } \\
\text { serious or life- } \\
\text { threatening reactions } \\
\text { such as cardiac } \\
\text { arrhythmias with } \\
\text { pimozide, so it is not } \\
\text { recommended for use } \\
\text { concomitantly }\end{array}$ \\
\hline
\end{tabular}

a NNRTIs may inhibit CYP3A4 and may decrease the metabolism of these drugs, which can cause increased plasma concentrations and toxicity. In turn, these drugs, also due to CYP3A4 inhibition, may decrease the metabolism of NNRTIs and increase plasma concentrations and toxicity.

Table 5. Clinical relevant bidirectional drug interactions mediated by enzyme inhibition (induction in some cases) of NNRTIs with other known enzyme inhibitors (Brophy et al., 2000; Jain et al., 2002; Krikorian \& Rudorf, 2005; Piscitelli \& Gallicano, 2001; Polk et al., 1999; Ray et al., 2004; Robertson et al., 2005b; Sekar et al., 2008; Vogel et al., 2004; Vourvahis \& Kashuba, 2007; Young, 2005)

lopinavir steady state concentration. (Dailly et al., 2005; Solas et al., 2004) The use of another low-dose PI as a pharmacokinetic extension agent (boosted), for instance ritonavir, is a strategy to counter this problem. (Acosta, 2002; Rathbun \& Rossi, 2002; Zeldin \& Petruschke, 2004) When using atazanavir with efavirenz it is recommended to add $100 \mathrm{mg}$ of ritonavir to the usual dose $(300 \mathrm{mg} / 24$ hours $)$. For lopinavir/ritonavir and efavirenz, lopinavir/ritonavir should be increased from 400/100 mg/12 hours (3 capsules) to 533/133 $\mathrm{mg} / 12$ hours (4 capsules) without change in the efavirenz dose. (DHHS, 2011; Solas et al., 2004; Dailly et al., 2005). For pediatric patients, in order to compensate the induction of efavirenz hepatic metabolism, it is suggested to increase the lopinavir/ritonavir dose to $300 / 75 \mathrm{mg} / \mathrm{m}^{2}$ twice daily. (Dailly et al., 2006) Related to this topic, some studies have shown that cimetidine may be useful as a pharmacokinetic extension agent (effect similar to ritonavir) when combined with saquinavir. (Boffito et al., 2002)

Besides the effect of efavirenz and nevirapine on PIs levels, these ITINNs, (Dejesus et al., 2006) efavirenz (mainly) and nevirapine may increase metabolism, lower levels and cause therapeutic failure with various medications, such as statins (Gerber et al., 2005) and warfarin. In table 6 the most relevant interactions due to enzyme induction mediated by these two NNRTIs are included. 


\begin{tabular}{|c|c|c|c|c|}
\hline \multirow{2}{*}{$\begin{array}{l}\text { Drug group or } \\
\text { drugs affected }\end{array}$} & \multicolumn{3}{|c|}{ Clinical relevance: level } & \multirow[t]{2}{*}{ Comments and suggestions } \\
\hline & Delavirdine & Efavirenz & Etravirine & \\
\hline $\begin{array}{l}\text { Protease inhibitors } \\
\text { (PI) }{ }^{a} \\
\text { - Indinavir (IDV) } \\
\text { (Harris et al., } \\
\text { 2006b) } \\
\text { - Nelfinavir (NFV) } \\
\text { - Ritonavir (RTV) } \\
\text { - Amprenavir } \\
\text { (APV) } \\
\text { - Tipranavir (TPV) } \\
\text { - Lopinavir/Riton } \\
\text { avir (LPV/r) } \\
\text { (Bergshoeff et al., } \\
\text { 2005; Dailly et al., } \\
\text { 2005; Solas et al., } \\
\text { 2004) }\end{array}$ & 2: high risk & 2: high risk & 2: high risk & $\begin{array}{l}\text { In general, the use of a single PI } \\
\text { with efavirenz (specially) or } \\
\text { nevirapine should be avoided. } \\
\text { Efavirenz may decrease by } 39 \% \\
\text { the minimum concentration of } \\
\text { lopinavir. The dose should be } \\
\text { increased to } 533 / 133 \mathrm{mg} / 12 \\
\text { hours }\end{array}$ \\
\hline $\begin{array}{l}\text { Saquinavir (SAQ) } \\
\text { (Boffito et al., 2002) }\end{array}$ & $\begin{array}{l}\text { 4: low risk } \\
\text { (Fletcher et } \\
\text { al., 2000) }\end{array}$ & $\begin{array}{l}\text { 3: medium } \\
\text { risk (Baker, } \\
1998)\end{array}$ & 2: high risk & $\begin{array}{l}\text { Concomitant use of etravirine } \\
\text { with saquinavir causes a } \\
\text { decrease of the saquinavir } \\
\text { plasma concentrations, due to } \\
\text { etravirine induction effect on } \\
\text { CYP3A4. (Etravirine, 2006; } \\
\text { Harris et al., 2006b) }\end{array}$ \\
\hline $\begin{array}{l}\text { Atazanavir (ATZ) } \\
\text { (Mullin et al., 2004) }\end{array}$ & 2: high risk & 2: high risk & $\begin{array}{l}\text { 1: Very } \\
\text { high risk }\end{array}$ & $\begin{array}{l}\text { It is recommended to add } 100 \\
\text { mg of ritonavir. } \\
\text { The plasma concentration of } \\
\text { atazanavir is reduced by } 38 \% \text { if } \\
\text { it is administered concomitantly } \\
\text { with etravirine. } \\
\text { Recommendation: Concomitant } \\
\text { use of etravirine with } \\
\text { atazanavir/ritonavir. (Aber, } \\
2008 \text { ) }\end{array}$ \\
\hline $\begin{array}{l}\text { Fosamprenavir } \\
(\mathrm{f}-\mathrm{APV}) \text { ( Back et } \\
\text { al, 2003) }\end{array}$ & 2: high risk & 2: high risk & $\begin{array}{l}\text { 1: Very } \\
\text { high risk }\end{array}$ & $\begin{array}{l}\text { Nevirapine may decrease } \\
\text { plasma concentrations between } \\
25 \text { and } 35 \% \\
\text { Fosamprenavir plasma } \\
\text { concentration is decreased by } \\
77 \% \text { if it is administered } \\
\text { concomitantly with etravirine. } \\
\text { Recommendation: avoiding } \\
\text { concomitant administration of }\end{array}$ \\
\hline
\end{tabular}




\begin{tabular}{|c|c|c|c|c|}
\hline & & & & $\begin{array}{l}\text { etravirine with fosamprenavir. } \\
\text { (Holdich et al., 2007) }\end{array}$ \\
\hline Darunavir & $\begin{array}{l}\text { No } \\
\text { information } \\
\text { available }\end{array}$ & 2: high risk & $\begin{array}{l}\text { No } \\
\text { information } \\
\text { available }\end{array}$ & $\begin{array}{l}\text { Efavirenz may decrease plasma } \\
\text { concentrations of darunavir } \\
\text { (Schöller et al., 2007) }\end{array}$ \\
\hline $\begin{array}{l}\text { Statins }{ }^{a} \text { (Gerber et } \\
\text { al., 2005; Sekar et } \\
\text { al., 2007a) } \\
\text { - Simvastatin } \\
\text { - Atorvastatin } \\
\text { - Pravastatin } \\
\text { - Lovastatin } \\
\text { - Rosuvastatin }\end{array}$ & $\begin{array}{l}\text { 3: medium } \\
\text { risk }\end{array}$ & $\begin{array}{l}\text { 3: medium } \\
\text { risk }\end{array}$ & $\begin{array}{l}\text { 3: medium } \\
\text { risk }\end{array}$ & $\begin{array}{l}\text { Possible reduction effect of these } \\
\text { hypolipidemic. Pharmacological } \\
\text { response should be traced and } \\
\text { dose adjusted, where necessary is } \\
\text { recommended to monitor } \\
\text { parameters the effectiveness of } \\
\text { lovastatin (lipid profile) and if } \\
\text { necessary make an adjustment in } \\
\text { medication dosage }\end{array}$ \\
\hline $\begin{array}{l}\text { Methadone (Altice } \\
\text { et al., 1999; Bruce et } \\
\text { al., 2006; Clarke et } \\
\text { al., 2001; Stocker et } \\
\text { al., 2004) }\end{array}$ & 2: high risk & 2: high risk & $\begin{array}{l}\text { No } \\
\text { information } \\
\text { available }\end{array}$ & $\begin{array}{l}\text { See text: methadone and } \\
\text { efavirenz (nevirapine) }\end{array}$ \\
\hline Warfarina ${ }^{a}$ & $\begin{array}{l}\text { 3: medium } \\
\text { risk }\end{array}$ & $\begin{array}{l}\text { 3: medium } \\
\text { risk }\end{array}$ & $\begin{array}{l}\text { No } \\
\text { information } \\
\text { available }\end{array}$ & Monitoring INR \\
\hline $\begin{array}{l}\text { Ethinyl estradiol a } \\
\text { (oral contraceptives) } \\
\text { (Mildvan et al, 2002) }\end{array}$ & 2: high risk & 2: high risk & $\begin{array}{l}\text { No } \\
\text { information } \\
\text { available }\end{array}$ & $\begin{array}{l}\text { Additional contraceptive } \\
\text { method should be used (barrier } \\
\text { method, for example) }\end{array}$ \\
\hline $\begin{array}{l}\text { Valproic acid } \\
\text { (Saraga et al., 2006) }\end{array}$ & 2: high risk & $\begin{array}{l}\text { No } \\
\text { information } \\
\text { available }\end{array}$ & $\begin{array}{l}\text { No } \\
\text { information } \\
\text { available }\end{array}$ & $\begin{array}{l}\text { Efavirenz may decrease } \\
\text { valproate concentrations by } \\
\text { induction of glucoronosil } \\
\text { diphosphate transferase }\end{array}$ \\
\hline $\begin{array}{l}\text { Macrolides } \\
\text { - Clarithromycin } \\
\text { (Schöller et al., } \\
\text { 2006b) } \\
\text { - Erythromycin }\end{array}$ & $\begin{array}{l}\text { No } \\
\text { information } \\
\text { available }\end{array}$ & $\begin{array}{l}\text { No } \\
\text { information } \\
\text { available }\end{array}$ & 2: high risk & $\begin{array}{l}\text { Etravirine may decrease the } \\
\text { drugs plasma concentrations, } \\
\text { because it exerts on enzyme } \\
\text { induction of CYP3A4 }\end{array}$ \\
\hline Maraviroc & $\begin{array}{l}\text { No } \\
\text { information } \\
\text { available }\end{array}$ & 2: high risk & $\begin{array}{l}\text { 3: medium } \\
\text { risk }\end{array}$ & $\begin{array}{l}\text { Efavirenz is an inducer of } \\
\text { CYP3A4 metabolism which can } \\
\text { lead to reduced plasma } \\
\text { concentrations of maraviroc. } \\
\text { Recommendation: In the } \\
\text { presence of enzyme inducers } \\
\text { such as efavirenz, maraviroc } \\
\text { may be increased to } 600 \mathrm{mg}\end{array}$ \\
\hline
\end{tabular}




\begin{tabular}{|c|c|c|c|c|}
\hline & & & & $\begin{array}{l}\text { twice daily (Abel et al., 2008) } \\
\text { Simultaneous administration of } \\
\text { etravirine and maraviroc causes } \\
\text { a decrease in plasma } \\
\text { concentrations of maraviroc } \\
\text { (Cmin decreased } 29 \% \text {, Cmax by } \\
60 \% \text { and AUC by } 53 \% \text { ). } \\
\text { Recommendation: dose } \\
\text { adjustment (Davis et al., 2007) }\end{array}$ \\
\hline $\begin{array}{l}\text { NNRTI } \\
\text { • Efavirenz } \\
\text { - Nevirapine }\end{array}$ & $\begin{array}{l}\text { No } \\
\text { information } \\
\text { available }\end{array}$ & $\begin{array}{l}\text { No } \\
\text { information } \\
\text { available }\end{array}$ & 2: high risk & $\begin{array}{l}\text { Concomitant use of etravirine } \\
\text { with nevirapine and efavirenz } \\
\text { can cause a decrease in plasma } \\
\text { concentrations of etravirine and } \\
\text { its therapeutic effect (Kakuda et } \\
\text { al., 2006) }\end{array}$ \\
\hline Raltegravir & $\begin{array}{l}\text { No } \\
\text { information } \\
\text { available }\end{array}$ & 2: high risk & 2: high risk & $\begin{array}{l}\text { Concomitant administration of } \\
\text { etravirine and raltegravir with } \\
\text { efavirenz can cause a decrease } \\
\text { in plasma concentration of } \\
\text { raltegravir. Cmin is reduced by } \\
34 \% \text { and AUC by } 10 \% \text {. } \\
\text { (Anderson et al., 2008; Menard } \\
\text { et al., 2009; Wittkop et al., 2009) }\end{array}$ \\
\hline Sildenafil & $\begin{array}{l}\text { No } \\
\text { information } \\
\text { available }\end{array}$ & $\begin{array}{l}\text { No } \\
\text { information } \\
\text { available }\end{array}$ & $\begin{array}{l}\text { 3: medium } \\
\text { risk }\end{array}$ & $\begin{array}{l}\text { Coadministration of etravirine } \\
\text { with sildenafil can cause a } \\
\text { decrease in sildenafil } \\
\text { concentrations by } 57 \% \text {. } \\
\text { Recommendation: } \\
\text { dose adjustment of sildenafil } \\
\text { Pérez et al., 2009) }\end{array}$ \\
\hline Buprenorphine & $\begin{array}{l}\text { No } \\
\text { information } \\
\text { available }\end{array}$ & $\begin{array}{l}\text { No } \\
\text { information } \\
\text { available }\end{array}$ & 2: high risk & $\begin{array}{l}\text { Coadministration of efavirenz } \\
\text { with buprenorphine may } \\
\text { decrease plasma concentrations } \\
\text { of its active metabolite, } \\
\text { norbuprenorphine, due to the } \\
\text { inductive effect exerted by the } \\
\text { efavirenz on CYP3A4 } \\
\text { (McCance-Katz et al., 2006) }\end{array}$ \\
\hline
\end{tabular}

a Efavirenz, in particular, and nevirapine can primarily induce CYP3A4 and increase the metabolism of these drugs, which can cause a decrease plasma concentrations and therapeutic effects.

Table 6. Clinical relevant drug interactions due to enzyme induction mediated by NNRTIs (DeJesus et al., 2006; DHHS, 2011; Krikorian \& Rudorf, 2005; Young, 2005) 
Methadone and efavirenz (nevirapine). Efavirenz and nevirapine may reduce methadone area under curve (AUC) by $57 \%$ and $46 \%$, respectively. (Altice et al., 1999) Therefore, when it is necessary to use methadone in patients that are using these NNRTIs, it is advisable to gradually increase the opiate dose from 10 to $10 \mathrm{mg}$, (Stocker et al., 2004) adjusting it based on effectiveness and safety parameters. Particular attention must be paid to withdrawal clinical manifestations. Withdrawal manifestations usually appear 7-10 days after initiating treatment with NNRTI and must not be confused with neurological toxicity symptoms associated with efavirenz (dizziness, headache, insomnia, concentration difficulty, nightmares, and agitation) that may occur within 1-2 days of starting treatment with efavirenz and which may be present during 14-28 days. (Bruce et al., 2006) Additionally, at sites with a suitable infrastructure, plasma levels of methadone can be monitored, aiming to reach a concentration of 400 and 250 micrograms/mL for (R, S)-methadone and (R) - methadone, respectively. (Bruce et al., 2006; Clarke et al., 2001; DHHS, 2011; Stocker et al., 2004)

Ritonavir as enzymatic inductor. Ritonavir, alone or in combination with other PIs, due to its ability to induce mainly glucuronyl-transferase and, to a lesser extent CYP2B6, which may produce ineffectiveness of some drugs:

- Ethinyl estradiol (oral contraceptive component). The contraceptive effect may be diminished when used simultaneously with ritonavir. (Ouellet et al., 1998) It is therefore advisable to inform the patient of the need to use a barrier method as a complementary birth control method (DHHS, 2011) (level 2: high risk).

- Thyroid hormones. It may cause metabolic inactivation of thyroid hormones and treatment failure (level 2: high risk). It is therefore recommended to monitor and adjust the levels of thyroid hormones in patients under treatment with ritonavir. (Touzot et al., 2006)

- Lamotrigine. It produces a decrease in anticonvulsant plasma levels, which can lead to ineffective treatment (level 2: high risk). It may be necessary to double lamotrigine dose in order to achieve therapeutic levels. (Van der Lee et al., 2006)

- Bupropion. It causes a decrease both in bupropion levels and its metabolite (hydroxybupropion), which could lead to ineffective treatment (Level 3: medium risk) and the need to double bupropión dose. (Hogeland et al., 2007) However, in theory, ritonavir can also act as an inhibitor of this isoenzyme and, therefore, increase concentrations of bupropion, so caution is advised until more data are available.

\subsection{Drug interactions mediated by known enzyme inducers and consequences on Pls and NNRTIs efficacy (Benator et al., 2007; DHHS, 2011; Hamzeh et al., 2003; Kraft et al., 2004; Krikorian \& Rudorf, 2005; Lim et al., 2004; Mullin et al., 2004; Romanelli \& Pomeroy, 2003; Young, 2005)}

The pharmacological effect of PIs and NNRTIs may be diminished and thus may appear therapeutic failure, associated with the emergence of resistance, when these ARV are combined or used simultaneously with known enzyme inducers (rifampicin, rifabutin, phenobarbital, primidone, carbamazepine, phenytoin), including St. John's wort. Rifabutin, a drug in the same family as rifampicin (rifamycins), is also characterized by inducing the metabolism of certain drugs, although to a lesser degree than rifampicin. (Finch et al., 2002)

\subsubsection{Interactions of rifamycins with protease inhibitors (PIs) or with non-nucleoside reverse transcriptase inhibitors (NNRTIs)}

Rifampicin and PIs. (Blumberg et al., 2003; Finch et al., 2002; Sekar et al., 2010; Spradling et al., 2002) In general, the use of rifampicin (rifabutin is preferred due to their lesser inductive 
effect than rifabutin) with a single PI, including the most recent, such as amprenavir and atazanavir, is considered contraindicated (level 1: very high risk), because in most cases, rifampicin produces non-efficacy PIs concentrations. Besides, most treatment guidelines for HIV/AIDS patients extend this contraindication even to PIs combined with ritonavir used as a pharmacokinetic extension agent (enhancer). (DHHS, 2011) However, for saquinavir, (Rolla et al., 2006) atazanavir, (Burger et al., 2006) and lopinavir, (La Porte et al., 2004) some studies show that adding ritonavir may counteract such effect in to some extent. In these cases, both saquinavir/ritonavir dose must be adjusted to 400/400 mg twice daily, (Aaron et al., 2004; de Jong et al., 2004; Rolla et al., 2006;) and lopinavir/ritonavir to $400 / 400 \mathrm{mg} / 12$ hours or to $800 / 200 \mathrm{mg} / 12$ hours. (Aaron et al., 2004; de Jong et al., 2004; La Porte et al., 2004)

Regarding the concomitant administration of saquinavir/ritonavir (1000/100 mg once daily) with rifampicin, because of some reports of liver toxicity, it is recommended to avoid such a combination. (Kashuba, 2005) Additionally, some studies show that in order to achieve saquinavir adequate levels, when saquinavir is used in combination with ritonavir, it is advisable to use doses higher than $1.600 / 200 \mathrm{mg} /$ day of this combination; (Ribera et al., 2007) however this situation could further increase the likelihood of liver toxicity problems. With respect to the advisable rifampicin dose (despite the possible inhibitory effect of PI), changes are not recommended, but liver functioning must be monitored. (La Porte et al., 2004; de Jong et al., 2004; Aaron et al., 2004) For amprenavir (Polk et al., 2001) even with low-dose ritonavir (100 or $200 \mathrm{mg} /$ day), their use in combination with rifampicin is contraindicated (level 1: very high risk). (DHSS, 2011)

Rifampicin and NNRTI. (Finch et al., 2002; de Jong et al., 2004; Ribera et al., 2001; Ramachandran et al., 2006; Weiner et al., 2005) In general, the use of rifampicin together with delavirdine is considered contraindicated (Borin et al., 1997) (level 1: very high risk). When it needs to use simultaneously rifampin with efavirenz, it is recommended to increase the NNRTI dose from 600 to $800 \mathrm{mg} /$ day. (DHHS, 2011; Matteelli et al., 2007) However, some studies conducted with patients weighing $50 \mathrm{~kg}$ in Thailand and infected with HIV and tuberculosis, treated with rifampicin and HAART, indicate that the use of the standard efavirenz dose $(600 \mathrm{mg} /$ day) may be enough to reach the desired levels and effects with NNRTI at 24 and 48 weeks. (Manosuthi et al., 2005, 2006) Similarly, results achieved in African patients support the use of efavirenz $600 \mathrm{mg} /$ day. (Friedland et al., 2006) Additionally, there are some reports of toxicity with efavirenz at doses of $800 \mathrm{mg} /$ day. (Brennan et al., 2005) Therefore, it is believed that with black patients weighing less than 55 $\mathrm{kg}$, as well as with Hispanic or Asian patients who show evidence of susceptibility to efivarenz toxic effects, it is recommended to use a $600 \mathrm{mg} /$ day dose of this NNRTI. (Matteelli et al., 2007) Thus, when it is indicate to use rifampicin with efavirenz no adjustments are recommended in the dose of rifampicin.

Consequently, in general: a) for patients receiving HAART, rifabutin should preferred to the use of for rifampicin, due to its significantly lower enzyme-inducing effect, b) the use of single PIs with rifampicin should be avoided; c) some studies show that rifampicin could be used with saquinavir, atazanavir, and lopinavir enhanced with ritonavir, but it is needing more evidence on the effectiveness and safety of such combinations, d) the use of delavirdine in conjunction with rifampicin or rifabutin is considered absolutely contraindicated, and e) in most cases where rifabutin or rifampicin is combined with PIs, dosage must be adjusted and potential toxicity must be monitored, particularly liver toxicity due to rifamycins. 
Hypericum (St. John's wort) as enzyme inducer and ARV drugs. In general, the combination of this herbal antidepressant with PIs or with NNRTIs, due to high probability of generating therapeutic failure, is considered contraindicated (level 1: very high risk). Thus, there are several reports of therapeutic failure of ritonavir and nevirapine, associated with the use of this herbal product. (Izzo, 2004; Winston \& Boffito, 2005; Zhou et al., 2004) This substance may induce both the systemic metabolism by CYP3A4, and the presystemic metabolism by intestinal or hepatic CYP3A4; additionally it may induce P-gp activity (bioavailability related interactions). (Lee et al., 2006)

Table 7 contains details of interactions produced by rifamycins (rifampin and rifabutin), and table 8 contains detailed information on interactions mediated by traditional anticonvulsants.

\subsection{Drug interactions related to bioavailability}

\subsubsection{Drug interactions related to changes in gastrointestinal $\mathrm{pH}$}

In general, an increased gastrointestinal $\mathrm{pH}$ may affect the amount absorbed of delavirdine, indinavir, fosamprenavir, tipranavir, (Vourvahis \& Kashuba, 2007) and atazanavir. (DeSilva et al., 2001; DHHS, 2011; Tomilo et al., 2006) However, it is basic to clarify some aspects:

- Concomitant administration of atazanavir with proton-pump inhibitors (omeprazole, lansoprazole, pantoprazole) (Tomilo et al., 2006) is not recommended (Level 2: high risk). The use of delavirdine with $\mathrm{H}_{2}$ antihistamines (cimetidine, ranitidine, famotidine, and nizatidine) and with proton-pump inhibitors (level 2: high risk) is also not recommended, due to a decrease near to $90 \%$ of the amount absorbed and, consequently, to a reduction in antiretroviral plasma concentrations, which can lead to treatment failure. (DHHS, 2011) In the case of atazanavir, famotidine can be used as an alternative to omeprazole, spacing its administration about 10 hours. A similar result can be achieved by adding low-dose ritonavir $(100 \mathrm{mg})$ at $300 \mathrm{mg} /$ day atazanavir, or by increasing the dose to $400 \mathrm{mg} /$ day of atazanavir. (Kashuba, 2005)

Combined administration of antacids does not seem to significantly affect the amount absorbed of fosamprenavir, (Ford et al., 2005) or raltegravir; however it is recommended to separate the taking of the two drugs when they are used in pharmacological therapy. (Kiser et al., 2010)

\begin{tabular}{|c|c|c|c|}
\hline \multirow[t]{2}{*}{ Drug group or drugs affected } & \multicolumn{2}{|c|}{$\begin{array}{c}\text { Clinical relevance: } \\
\text { level }\end{array}$} & \multirow{2}{*}{$\begin{array}{l}\text { Comments and suggestions } \\
\text { (see text: rifampicin and PI } \\
\text { and rifampicin and NNRTI) }\end{array}$} \\
\hline & Rifampicin & Rifabutin & \\
\hline $\begin{array}{l}\text { PIs }{ }^{a} \text { (Aaron et al., 2004; Benator et al., } \\
\text { 2007; Blumberg et al., 2003; Burger et } \\
\text { al., 2006; DeJong et al. 2004; Finch et } \\
\text { al., 2002; Hamzeh et al., 2003; Kraft et } \\
\text { al., 2004; La Porte et al., 2004; Polk et } \\
\text { al., 2001; Ribera et al., 2007; Rolla et } \\
\text { al., 2006; Spradling et al., 2002) } \\
\text { Ritonavir (Aaron et al., 2004; } \\
\text { Blumberg et al., 2003; DeJong et al. } \\
\text { 2004; Finch et al., 2002; Spradling et } \\
\text { al., 2002) }\end{array}$ & $\begin{array}{l}\text { 1: very } \\
\text { high risk }\end{array}$ & 2: high risk & $\begin{array}{l}\text { Adjust rifabutin dose } \\
150 \mathrm{mg} / 48 \text { hours, with no } \\
\text { change in ritonavir }\end{array}$ \\
\hline
\end{tabular}




\begin{tabular}{|c|c|c|c|}
\hline $\begin{array}{l}\text { Indinavir (Hamzeh et al., 2003, Kraft } \\
\text { et al., 2004) }\end{array}$ & $\begin{array}{l}\text { 1: very } \\
\text { high risk }\end{array}$ & 2: high risk & $\begin{array}{l}\text { Adjust the dose of rifabutin } \\
150 \mathrm{mg} / 24 \text { hours and } \\
\text { indinavir at } 1,000 \mathrm{mg} / 8 \\
\text { hours }\end{array}$ \\
\hline $\begin{array}{l}\text { Saquinavir (Aaron et al., 2004; } \\
\text { Blumberg et al., 2003; DeJong et al. } \\
\text { 2004; Finch et al., 2002; Ribera et al., } \\
\text { 2007; Rolla et al., 2006; Spradling et } \\
\text { al., 2002) }\end{array}$ & $\begin{array}{l}\text { 1: very } \\
\text { high risk }\end{array}$ & 2: high risk & $\begin{array}{l}\text { Use usual dose of rifabutin } \\
(300 \mathrm{mg} / \text { day), with no } \\
\text { change in saquinavir }\end{array}$ \\
\hline $\begin{array}{l}\text { Nelfinavir (Aaron et al., 2004; } \\
\text { Benator et al., 2007; Blumberg et al., } \\
\text { 2003; DeJong et al. 2004; Finch et al., } \\
\text { 2002; Spradling et al., 2002) }\end{array}$ & $\begin{array}{l}\text { 1: very } \\
\text { high risk }\end{array}$ & 2: high risk & $\begin{array}{l}\text { Adjust rifabutin dose } 150 \\
\text { mg/24 hours and nelfinavir } \\
\text { to } 1,000 \mathrm{mg} / 8 \text { hours }\end{array}$ \\
\hline Atazanavir (Burger et al., 2006) & $\begin{array}{l}\text { 1: very } \\
\text { high risk }\end{array}$ & 2: high risk & $\begin{array}{l}\text { Adjust rifabutin dose } 150 \\
\text { mg/48hours, with no change } \\
\text { in atazanavir }\end{array}$ \\
\hline Amprenavir (Polk et al., 2001) & $\begin{array}{l}\text { 1: very } \\
\text { high risk }\end{array}$ & 2: high risk & $\begin{array}{l}\text { Adjust rifabutin dose } 150 \\
\text { mg/24 hours, with no } \\
\text { change in amprenavir }\end{array}$ \\
\hline $\begin{array}{l}\text { Fosamprenavir (Aaron et al., 2004; } \\
\text { DeJong et al. 2004) }\end{array}$ & $\begin{array}{l}\text { 1: very } \\
\text { high risk }\end{array}$ & 2: high risk & $\begin{array}{l}\text { Adjust rifabutin dose } 150 \\
\text { mg/24 hours, with no } \\
\text { changes in fosamprenavir } \\
\text { dose }\end{array}$ \\
\hline $\begin{array}{l}\text { Tipranavir (Vourvahis \& Kashuba, } \\
\text { 2007) }\end{array}$ & $\begin{array}{l}\text { 1: very } \\
\text { high risk }\end{array}$ & 2: high risk & $\begin{array}{l}\text { Adjust rifabutin dose } 150 \\
\text { mg/48hours, with no } \\
\text { changes in tipranavir dose } \\
\end{array}$ \\
\hline $\begin{array}{l}\text { Lopinavir/ritonavir (Aaron et al., } \\
\text { 2004; La Porte et al., 2004; Spradling } \\
\text { et al., 2002) }\end{array}$ & $\begin{array}{l}\text { 1: very } \\
\text { high risk }\end{array}$ & 2: high risk & $\begin{array}{l}\text { Adjust rifabutin dose } 150 \\
\mathrm{mg} / 24 \text { hours, with no } \\
\text { changes in } \\
\text { lopinavir/ritonavir dose }\end{array}$ \\
\hline Darunavir & 2: high risk & 2: high risk & $\begin{array}{l}\text { The predominant metabolite } \\
\text { of rifabutin (RFB) is 25-O- } \\
\text { desacetylrifabutin (desRFB), } \\
\text { darunavir by inhibition of } \\
\text { CYP3A4 may increase } \\
\text { plasma concentrations of } \\
\text { RFB, as well as its side effects } \\
\text { (Sekar et al., 2010) } \\
\text { Rifampin may significantly } \\
\text { decrease the plasma } \\
\text { concentrations of darunavir } \\
\text { (Tibotec, 2008) }\end{array}$ \\
\hline $\begin{array}{l}\text { Non-nucleosid transcriptase inhibitors } \\
\left(\text { NNRTI) }{ }^{a} \quad \text { (DeJong et al. 2004; Finch }\right. \\
\text { et al., 2002; Friedland et al., 2006; } \\
\text { Manosuthi et al., 2005, 2006; } \\
\end{array}$ & $\begin{array}{l}\text { 1: very } \\
\text { high risk }\end{array}$ & $\begin{array}{l}\text { 1: very high } \\
\text { risk }\end{array}$ & $\begin{array}{l}\text { This combination is } \\
\text { considered absolutely } \\
\text { contraindicated }\end{array}$ \\
\hline
\end{tabular}


McCance-Katz et al., 2006; Matteelli et al., 2007a; Ramachandran et al., 2006; Ribera et al., 2001)

Delavirdine (Borin et al., 1997;

DeJong et al. 2004; McCance-Katz et al., 2006; Spradling et al., 2002)

Nevirapine (Benator et al., 2007; Blumberg et al., 2003; Borin et al., 1997; Burger et al., 2006; Hamzeh et al., 2003; Kraft et al., 2004; La Porte et al., 2004; Polk et al., 2001; Ramachandran et al., 2006; Ribera et al., 2001, 2007; Rolla et al., 2006)

Efavirenz (Brennan et al., 2005; DeJong et al. 2004, Friedland et al., 2006; Manosuthi et al., 2005, 2006; Matteelli et al., 2007a, 2007b; Weiner et al., 2005)

\begin{tabular}{|l|l|l|l|}
\hline Etravirine (Abel et al., 2008) & 2: high risk & $\begin{array}{l}3: \text { medium } \\
\text { risk }\end{array}$ & $\begin{array}{l}\text { Co-administration of } \\
\text { etravirine with rifampicin } \\
\text { causes a significant decrease } \\
\text { in plasma concentrations of } \\
\text { etravirine, due to inductor } \\
\text { effect on CYP3A4 of } \\
\text { rifampicin } \\
\text { Co-administration of } \\
\text { etravirine with rifabutin } \\
\text { causes a decrease by 37\% of } \\
\text { plasma concentrations of } \\
\text { etravirine }\end{array}$ \\
\hline $\begin{array}{l}\text { Entry inhibitor: } \\
\text { Maraviroc (Ogbuokiri, 2009) }\end{array}$ & 2 high risk & $\begin{array}{l}\text { 3: medium } \\
\text { risk }\end{array}$ & $\begin{array}{l}\text { These drugs can reduce the } \\
\text { maraviroc plasma } \\
\text { concentrations by } 66 \% . \\
\text { Recommendation: dose } \\
\text { adjustment of maraviroc to } \\
600 \text { mg }\end{array}$ \\
\hline
\end{tabular}

a Rifampicin $\rightarrow$ rifabutin can induce CYP3A4 and increase the metabolism of these drugs, which can cause a decrease in plasma concentrations and virological response. In turn, some PIs or NNRTIs may modify the metabolism and concentrations or rifamycins, particularly by inhibition of CYP3A4, which can decrease their metabolism and increase plasma and concentrations and toxic effects of rifampicin and rifabutin, particularly liver and blood effects.

Table 7. Clinical relevant drug interactions mediated by rifampicin and rifabutin (DHHS, 2011; Tran et al., 2001) 


\begin{tabular}{|c|c|c|c|c|}
\hline \multirow{2}{*}{$\begin{array}{l}\text { Drug group or } \\
\text { drugs affected }\end{array}$} & \multicolumn{3}{|c|}{ Clinical relevance: level } & \multirow[t]{2}{*}{ Comments and suggestions } \\
\hline & $\begin{array}{l}\text { Phenobarbital } \\
\text { and primidone }\end{array}$ & Phenytoin & Carbamazepine & \\
\hline \multicolumn{5}{|c|}{ Protease Inhibitors (PI) a (Lim et al, 2004; Mullin et al., 2004; Romanelli \& Pomeroy, 2003) } \\
\hline - Ritonavir & 2: high risk & 2: high risk & 2: high risk & \multirow{8}{*}{$\begin{array}{l}\text { Traditional anticonvulsants } \\
\text { (except valproic acid) can } \\
\text { cause ineffectiveness of PI } \\
\text { (including their combination } \\
\text { with low dose ritonavir). } \\
\text { (Ogbuokiri, 2009) } \\
\text { It is recommended the use of } \\
\text { second-generation } \\
\text { anticonvulsants (gabapentine, } \\
\text { lamotrigine, vigabatrin and } \\
\text { topiramate). } \\
\text { Gabapentine, due to its } \\
\text { pharmacological properties (it } \\
\text { is not metabolized, it does not } \\
\text { affect CYP activity), is } \\
\text { considered the best option } \\
\text { (Mullin et al., 2004; Romanelli } \\
\& \text { Pomeroy, 2003) }\end{array}$} \\
\hline - Indinavir & 2: high risk & 2: high risk & 2: high risk & \\
\hline - Saquinavir & 2: high risk & 2: high risk & 2: high risk & \\
\hline - Nelfinavir & 2:high risk & 2:high risk & 2:high risk & \\
\hline - Atazanavir & 2:high risk & 2:high risk & 2:high risk & \\
\hline - Amprenavir & 2:high risk & 2:high risk & 2:high risk & \\
\hline - Fosamprenavir & 2:high risk & 2:high risk & 2:high risk & \\
\hline - Tipranavir & 2:high risk & 2:high risk & 2:high risk & \\
\hline $\begin{array}{l}\text { - Lopinavir/ } \\
\text { ritonavir }\end{array}$ & 2:high risk & 2:high risk & 2:high risk & $\begin{array}{l}\text { Lopinavir / ritonavir may } \\
\text { decrease the plasma } \\
\text { concentrations of phenytoin, } \\
\text { due to the induction of } \\
\text { CYP2C9 }\end{array}$ \\
\hline \multicolumn{5}{|c|}{$\begin{array}{l}\text { Non-nucleoside transcriptase inhibitors (NNRTI) a (Mullin et al., 2004; Romanelli \& Pomeroy, } \\
\text { 2003; Tran et al., 2001; Tseng E Foisy, 1999) }\end{array}$} \\
\hline - Delavirdine & $\begin{array}{l}\text { 1: very high } \\
\text { risk }\end{array}$ & $\begin{array}{l}\text { No } \\
\text { information } \\
\text { available }\end{array}$ & $\begin{array}{l}\text { 1:very high } \\
\text { risk }\end{array}$ & \multirow{3}{*}{$\begin{array}{l}\text { Delavirdine with any of the } \\
\text { traditional anticonvulsants } \\
\text { (except valproic acid) is } \\
\text { considered contraindicated. } \\
\text { Traditional anticonvulsants } \\
\text { (except valproic acid) can } \\
\text { cause NNRTI ineffectiveness } \\
\text { (Acosta, 2002) }\end{array}$} \\
\hline - Nevirapine & 2: high risk & $\begin{array}{l}\text { No } \\
\text { information } \\
\text { available }\end{array}$ & 2:high risk & \\
\hline - Efavirenz & 2:high risk & $\begin{array}{l}\text { No } \\
\text { information } \\
\text { available }\end{array}$ & 2: high risk & \\
\hline \multicolumn{5}{|l|}{ Entry inhibitor } \\
\hline $\begin{array}{l}\text { - Maraviroc } \\
\text { (Ogbuokiri, } \\
\text { 2009) }\end{array}$ & $\begin{array}{l}\text { 3: medium } \\
\text { risk }\end{array}$ & $\begin{array}{l}\text { 3: medium } \\
\text { risk }\end{array}$ & $\begin{array}{l}\text { 3: medium } \\
\text { risk }\end{array}$ & $\begin{array}{l}\text { Traditional anticonvulsants } \\
\text { (except valproic acid) can } \\
\text { cause ineffectiveness of } \\
\text { maraviroc }\end{array}$ \\
\hline
\end{tabular}

Table 8. Clinical relevant drug interactions mediated by traditional anticonvulsants (DHHS, 2011; Tran et al., 2001) 
- Etravirine-Ranitidine. The concurrently use of etravirine and ranitidine for a period of eight days may cause a reduction in absorption of etravirine due to decrease of gastric (Schöller-Gyüre et al., 2008) acidity (level 3: medium risk).

- Darunavir-Omeprazole/Ranitidine. Due to the absorption of some PIs is $\mathrm{pH}$ dependent gastric acid, antacids may inhibit absorption of PIs. However, some studies show that there are not clinically significant DIs between darunavir and omeprazole/ranitidine, thus no dose adjustment is required to concomitant administration. (Sekar et al., 2007b)

- Absorption of delavirdine is noticeably decreased at $\mathrm{pH}$ values below 3 . Therefore, its administration with antacids may cause a decrease on levels and effects (level 3: medium risk), and it is recommended to space its administration, at least an hour. (Tran et al., 2001)

Broadly, didanosine in buffered tablets may decrease absorption, levels and efficacy of delavirdine and indinavir (as well of other drugs that requiring an acidic environment for absorption), due to didanosine excipients maintain gastric $\mathrm{pH}$ above 3 . However, the addition of didanosine in a new dosage form (enteric-coated granules within capsules) minimizes this effect, as evidenced by the results of studies on drugs whose bioavailability may be decreased by interactions with antacids such as indinavir, ketoconazole, and ciprofloxacin. (Damle et al., 2002b) Furthermore, the buffered tablet effect cannot be generalized for drugs requiring an acidic $\mathrm{pH}$ for absorption, such as itraconazole or fluconazole, whose bioavailability is not altered when administered simultaneously with didanosine, regardless of dosage form used. (Damle et al., 2002a)

Related to this type of mechanism, in general, food (drug-food interactions) can decrease the amount absorbed and effects of several ARV drugs. For example, in the case of didanosine (buffered tablet and enteric-coated granules within capsules), it is recommended to take it on an empty stomach ( 2 hours before or two hours after meals), (la Porte et al., 2005) because a decrease (between 18 and 55\%) in the amount absorbed can be produced (level 3: risk medium). (Damle et al., 2002c; Kearney et al., 2005) Such effect may be minimized if didanosine is administered in combination with indinavir/ritonavir or with tenofovir (La Porte et al., 2005; Kearney et al., 2005) (see didanosine- tenofovir Interaction below).

\subsubsection{Interactions related to presystemic metabolism and/or P-glycoprotein activity}

P-gp (ABCB1) is one of the most extensively studied transporters regarding DIs. P-gp is expressed in multiple key organs in drug disposition such as small intestine, blood-brain barrier, kidney, and liver. Therefore, P-gp mediated DIs can occur at various organs and tissues. Thus, the induction or inhibition of P-gp can lead to drug-drug interactions. For instance, induction of the intestinal P-glycoprotein activity can cause reduced bioavailability of orally administered drugs and cause therapeutic failure. In contrast, the inhibition of the intestinal P-gp activity can lead to increased bioavailability, concentrations and adverse drug effects. (Josephson, 2010)

Broadly, PIs, particularly ritonavir, are mechanism-based intestinal P-gp efflux activity inhibition, which partially explains the effect on the levels and clinical results of some drugs, (de Maat et al., 2003) such as cyclosporine (Izzedine et al. 2004) and digoxin. (Penzak et al., 2004) Ritonavir can also inhibit renal P-gp activity, which may lead to a decrease in renal clearance of digoxin, associated with an increase in the levels and effects of this drug. (Ding et al., 2004) 
Simultaneous administration of saquinavir $500 \mathrm{mg}$ (low dose ritonavir) with omeprazole is associated with an increase of $82 \%$ in AUC of saquinavir. Although the mechanism is unclear, it is believed that this effect is due to the fact that omeprazole can inhibit P-gp activity and other membrane transporters activity. (Winston et al., 2006) Similarly, saquinavir bioavailability increases significantly when administered with cimetidine, (Boffito et al., 2002) ranitidine or food, an effect independent of $\mathrm{pH}$ increase. (Kakuda \& Falcon, 2006) These findings could lead to believe that food is likely to increase solubility and to decrease the hepatic first pass effect, while the effect of cimetidine, ranitidine, and omeprazole, similar to what has been observed with other known inhibitors of intestinal and hepatic CYP3A4, may be associated to decreased presystemic metabolism and, to a lesser extent, to decreased P-gp intestinal activity. (Amariles, 2007)

\section{Clinically relevant pharmacodynamic drug interactions in HIV-infected patients receiving antiretroviral therapy}

Due to it is important some examples related to pharmacodynamic DIs in HIV-infected patients are detailed:

- Lamivudine and emtricitabine: this combination is considered inadequate, since these drugs show a minimum additive effect, as well as a similar profile of resistance (DHHS, 2011) (level 2: high risk).

- Stavudine and zidovudine: this combination is considered inadequate, because their chemical similarity can lead to competition for intracellular phosphorylation zidovudine inhibits phosphorylation and effects of stavudine (Ray, 2005) which might cause therapeutic failure (Level 2: high risk). Concerning this interaction, it is important to note that prior exposure to zidovudine ( 2 to 45 months) does not influence the ability of HIV-infected patients to phosphorylate stavudine. (Hoggard et al., 2001) Due to similar mechanism, the lamivudine and zalcitabine combination is considered inadequate, since lamivudine may inhibit the phosphorylation of zalcitabine, which may lead to therapeutic failure (Becher et al., 2004; DHHS, 2011; Havlir et al., 2000) (level 2: high risk).

- Abacavir, tenofovir, and lamivudine (or emtricitabine) as a triple NRTI therapy: This combination is considered contraindicated due to the rapid onset of virologic failure, when used as initial therapy (level 2: high risk). (DHHS, 2011) A similar consideration has been established for combination of tenofovir, didanosine, and lamivudine (emtricitabine). (DHHS, 2011)

- Amprenavir and fosamprenavir: This combination is not recommended, because fosamprenavir is a prodrug of amprenavir, thus their combined use increases the risk of adverse drug reactions without additional benefits (level 2: high risk).(DHHS, 2011)

- Stavudine and aminopterin: This combination is not recommended, because the combination causes an inhibition of hepatic mitochondrial DNA and hepatic toxicity (Setzer et al., 2008) (level 1: very high risk).

- Tenofovir and cisplatin or pemetrexed: The use of tenofovir in patients receiving cisplatin or pemetrexed may increase the risk of renal toxicity (3: medium risk). (Makinson et al., 2010)

- Tenofovir/emtricitabine, disulfiram and nifedipine: The use of nifedipine in HIVinfected patients receiving tenofovir/emtricitabine and disulfiram may cause lactic acidosis (level 2: high risk). ( Moling et al., 2009) 
- Zidovudine, cisplatin or pemetrexed: The use of zidovudine in patients receiving cisplatin or pemetrexed may increase the risk of hematological toxicity (Makinson et al., 2010) (level 3: medium risk).

- Broadly, the use of drugs with a similar unsafe profile leads to an increased probability and severity of adverse effects, for instance:

- Stavudine and didanosine: This combination is considered absolutely contraindicated (level 1: very high risk), due to increased likelihood of significant synergism of adverse drug problems associated with mitochondrial toxicity, which may manifest, especially as peripheral neuropathy, pancreatitis, and lactic acidosis. (Boubaker et al., 2001; Catanzaro et al., 2004; Coghlan et al., 2001; DHHS, 2011) Similarly, didanosine/zalcitabine and stavudine/zalcitabine combinations are considered contraindicated because of a greater likelihood and severity of peripheral neuropathy (Dalakas et al., 2001; Simpson \& Tagliati, 1995) (level 1: very high risk). In general, NRTIs (lower risk with abacavir and lamivudine) may inhibit competitively the mitochondrial DNA polymerase gamma, an enzyme responsible for repairing mitochondrial DNA associated with oxidative alterations. (Dagan et al., 2002) Therefore, the use of NRTI may lead to depletion of DNA and uncoupling of mitochondrial respiratory chain and thus, to the accumulation of radicals and free fatty acids, as well as dicarboxylic acids, responsible for mitochondrial toxicity associated with this group of drugs. (Petit et al, 2005)

- Zidovudine and ganciclovir: This combination increases the likelihood of developing bone marrow suppression, a condition associated with the occurrence of severe hematologic toxicity and life threatening infections, including the progression of cytomegalovirus infection (Hochster et al., 1990) (level 1 : very high risk). Related to this type of interaction, another combination with additive hematologic toxicity combination is zidovudine with ribavirin, associated with an increased risk of anemia (DHHS, 2011) (level 2: high risk).

- Didanosine and ribavirin. This combination increases the likelihood of mitochondrial toxicity, which can lead to lactic acidosis and pancreatitis (Fleischer et al., 2004; Perronne et al. 2006) (level 2: high risk). Similarly, the didanosine-adefovir combination increases the risk of pancreatitis (Perronne et al. 2006) (level 2: high risk).

- Atazanavir and indinavir: This combination increases the likelihood of developing hyperbilirubinemia (DHHS, 2011) (level 2: high risk).

- Drugs with additive hepatic toxicity: In general, concomitant use of hepatotoxic drugs enhances the probability of hepatic alterations, for instance, when rifampicin is used with isoniazid (Steele et al., 1991) or with pyrazinamide (Yee et al., 2003) for treatment of tuberculosis; or when acetaminophen is used with zidovudine (Shriner \& Goetz, 1992) (Level 2 or 3: high or middle risk).

- Drugs with additive renal toxicity: Simultaneous use of renal toxic drugs increases the likelihood of problems in this organ, for instance the simultaneously use of adefovir, acyclovir (intravenously), cidofovir, foscarnet, indinavir, ritonavir, tenofovir, pentamidine, aminoglycosides, and amphotericin B (Fisher et al., 1989) (level 2 or 3: high risk or middle).

\section{Clinically relevant drug-disease interactions in HIV-infected patients receiving antiretroviral treatment}

Due to it is important some examples related to clinically relevant drug-disease interactions in HIV-infected patients are detailed: 
- Pregnancy

- Amprenavir oral solution, due to the high content of propylene glycol, as an excipient, can cause toxicity problems (DHHS, 2011) (level 2: high risk).

- Efavirenz, especially during the first 3 months, because it represents a potential teratogenic risk, it is included in the D category of FDA classification of drugs for teratogenic risk (AIDS Patient Care, 2005; DHHS, 2011) (level 2: high risk).

- Didanosine and stavudine, because there are reports of severe and even fatal lactic acidosis in pregnant women receiving stavudine and didanosine (DHHS, 2011) (level 2: high risk).

- Moderate (Child Pugh score: 7-9 points) or severe (Child Pugh: over 9 points) liver failure

- $\quad$ NNRTI and PI. In general this class of drugs should be administered with caution or avoided altogether in patients with moderate or severe liver failure (DHHS, 2011) (level 3: medium risk).

- Amprenavir, fosamprenavir. Avoid their use in the presence of severe liver failure (Amariles et al., 2007c; DHHS, 2011) (level 3: medium risk).

- Amprenavir/ritonavir, tipranavir / ritonavir. Their use is contraindicated in severe liver failure (Amariles et al., 2007c; DHHS, 2011) (level 2: high risk).

- Children under 4 years. Amprenavir oral solution. Due to the high content of propylene glycol, as an excipient, it can cause toxicity problems (DHHS, 2011) (level 2: high risk).

- Women with CD4 lymphocyte counts $>250 \mathrm{cells} / \mathrm{mm}^{3}$ or men with CD4 lymphocyte counts $>400$ cells $/ \mathrm{mm}^{3}$. For these groups of patients there are reports of serious, sometimes fatal, liver impairments attributed to nevirapine (DHHS, 2011) (level 2: high risk).

- Cirrhosis. The combination of didanosine and ribavirin can lead to liver decompensation. Therefore, this combination is considered no-adequate for patients with advanced liver fibrosis (Perronne, et al. 2006) (level 2: high risk).

- HIV. The use of rifapentine (rifamycin) is considered inadequate, due to fewer efficacies in preventing the onset of tuberculosis in this group of patients. For this reason this could be considered as a possible drug-disease interaction (DHHS, 2011) (level 2: high risk).

\section{Other clinically relevant drug interactions in HIV-infected patients attributed to different or unclear mechanisms}

Other examples of clinically relevant DIs in HIV-infected patients attributed to different or unclear mechanisms are:

- Zidovudine and enzyme inducers. Zidovudine is metabolized and inactivated by glucuronyl transferase. Thus, inducers or inhibitors of this enzyme can affect its levels and effects (Kiang et al, 2005). For example, rifampicin may increase zidovudine metabolism and decrease its levels and effects. (Gallicano et al., 1999)

- Tenofovir and didanosine. Tenofovir increases didanosine levels and toxicity (particularly, pancreatitis), (Martinez et al., 2004) apparently due to inhibition of its metabolism by purine nucleoside phosphorylase. (Ray et al., 2004) It is thus recommended to reduce the dose from $400 \mathrm{mg}$ to $250 \mathrm{mg} / 24$ hours in patients weighing 
less than 60 kg. (Kearney et al., 2005; Martinez et al., 2004; Antoniou et al., 2003) Additionally, even using reduced dose, didanosine toxicity signs and symptoms should be monitored (significant increase in serum amylase or lipase, neuropathy, paresthesia, nausea, vomiting, and abdominal pain). (DHHS, 2011) There are reports of didanosine toxicity (deadly lactic acidosis and acute hepatic failure), (Guo \& Fung, 2004; Masía et al., 2005) at doses of $200 \mathrm{mg} /$ day, especially in women weighing 60 or less $\mathrm{kg}$. A similar effect could be observed when administering didanosine with other purine nucleoside phosphorylase inhibitory drugs, such as allopurinol, ganciclovir (Moling et al., 2009) and valganciclovir. (Tseng \& Salit, 2007) Additionally, the use of didanosine-tenofovir combination may lead to decrease in the CD4 lymphocyte levels, thus this combination should be avoided (Anderson \& Kakuda, 2006; Barreiro \& Soriano, 2006) (level 1 or 2: Very high risk" or higher). Similarly, the didanosine and ganciclovir combination has also been associated with decreased CD4 cell levels (Tseng \& Salit, 2007) (level 2: high risk).

- Amprenavir oral solution (propylene glycol) and oral ritonavir (ethanol excipient). Because propylene glycol and ethanol are metabolized by the same enzyme, accumulation and toxicity may occur. (DHHS, 2011) Also, related to the presence of excipients, the use of lopinavir/ritonavir oral solution (4.2\% in ethanol) combined with metronidazole or disulfiram, substances that may inhibit the alcohol dehydrogenase, may lead to the development of disulfiram effect. (Cvetkovic \& Goa, 2003; de Maat et al., 2003)

- Atazanavir and tenofovir. Tenofovir reduces atazanavir levels through a mechanism still to be established, so it is recommend using atazanavir $(300 \mathrm{mg})$ together with low dose ritonavir (100 mg) as an enhancing agent. (Taburet et al., 2004) In addition, lopinavir/ritonavir and atazanavir may increase levels and toxic renal effects of tenofovir. (Perronne et al., 2006) It is important to illustrate that the inductive effects of tenofovir on the metabolism of atazanavir have not been documented to others PIs (Boffito et al., 2005)

- Saquinavir and adefovir. Adefovir (antiviral used in the treatment of hepatitis B) lowers saquinavir levels and effects. (Perronne et al. 2006)

- Tenofovir and enzyme inducers. NRTIs with inducing capacity (efavirenz and nevirapine) (Droste et al., 2006) and probably rifampicin, (Droste et al., 2005) do not cause variation in tenofovir levels and effects. Therefore, it is not recommended to adjust the dose of either drug, when it is necessary to use such combinations.

- Amprenavir capsules, vitamin E, and warfarin levels. Amprenavir capsules contain an amount of vitamin $\mathrm{E}$ that exceeds recommended daily dose, so in patients treated with amprenavir in this dosage form, vitamin E supplementation should be avoided. In patients under treatment with warfarin, high levels of vitamin E (associated with intakes higher than 400 IU per day) increase the risk of bleeding. (Amsay et al, 2005, Heck et al., 2000)

- Strong or moderate inhibitors and PIs. Some known enzyme inhibitors such as ketoconazole may decrease the extra-intestinal P-gp efflux activity and increasing PIs levels and effects in certain body areas, such as the cerebrospinal fluid, which have been evidenced for ritonavir and saquinavir. (Cvetkovic \& Goa, 2003; Oldfield \& Plosker, 2006; Lin \& Yamazaki, 2003; Lin, 2003; Khaliq et al., 2000)

- Rosiglitazone and nevirapine. Rosiglitazone, apparently due to increased CYP3A4 or P-gp activity, may decrease nevirapine levels. This effect does not occur when using 
lopinavir/ritonavir with nevirapine. (Oette et al., 2005) Thus, it is recommended to monitor nevirapine levels and effects, when this drug is used in combination with rosiglitazone (level 2: high risk).

- Maraviroc and raltegravir. The concomitantly administration of these drugs can reduce peak concentrations of both drugs due to changes in pre-systemic elimination associated to changes in absorption and/or first pass metabolism; however, the exact mechanism of interaction has not been determined (Andrews et al., 2010) (3: medium risk).

\section{Software for evaluating and predicting clinical relevance of drug interactions in HIV-infected patients receiving antiretroviral therapy}

The identification, systematization, evaluation, and prediction of DIs may be easier by using computer applications. Additionally, these kinds of informatics tools may contribute to reduce the risk to arise of clinical relevant DIs, and thus, the negative effect in goals of HAAR or cART in patients with HIV/SIDA. Thus, the design software that facilitates the identification and prediction the clinical relevance of drug interactions may be an important contribution to get the possible outcomes best in HIV-infected patients receiving HAART or cART. (Amariles et al., 2008) In this way, the following tasks have been done:

a. Structured and systematic review of publications on Pubmed/Medline and other electronic databases, supplemented by other primary and secondary information sources to identify DIs in HIV-infected patients receiving ART therapy. Thus, published articles of DIs in HIV-infected patients were identified by a comprehensive literature search using electronic databases of information sources (Medline/Pubmed, SIETES, Medscape, and Tripdatabase) to identify all full text or abstracts published in English and Spanish from January 1996 to February 2011. Additionally, a search was done in some specify journals: New England Journal of Medicine, British Medical Journal, and other recognized information sources: the electronic sheet drug interactions: www.drug-interactions.com; Philip D. Hansten, John R. Horn. Managing Clinically Important Drug Interactions, facts and comparison, 2003; Stockley IH. Drug Interactions. First edition. Pharma Editores. Spain, 2004, and Drug Interaction Facts, Micromedex (Drug-REAX) computer program.

b. Classification of the clinical relevance of pairs of the identified DIs. The accessing and predicting the clinical relevance of pairs of the identified DIs were based on the severity and probability of occurrence of the DIs. Based on the possible combinations of severity and probability of occurrence, DIs were grouped into 4 categories: Level 1 (very high risk), level 2 (high risk), level 3 (medium risk), an level 4 (low risk) as it was mentioned in numeral 2.3 (Determining and predicting of the clinical relevance level) (Amariles et al., 2007a)

c. Software design. The results of the review and evaluation of clinical relevance of the DIs were used to design a platform with alert generator, report generator, constant evolution support systems data integrity, network management intranet and Internet Web and Windows platforms. The developed software facilitates the identification, evaluation, and prediction of clinical relevant of 1,082 drug pairs of potential DIs, near to $80 \%$ of them due to pharmacokinetic mechanism (changes in plasma concentration), mainly associated to enzyme inhibition. The scaling of these 1,082 drug pairs of the recognized DIs according to different dosage forms and strengths of identified drugs, generates a total of 6,087 pairs of DIs, which, according their clinical relevance, 4,158 
(68.3\%) are clinical relevant (Levels 1 and 2) in HIV-infected patients receiving ARV therapy. Thus, the software meets the requirements defined for this type of programs. (Gaikwad et al., 2007; Rodríguez et al., 2009)

d. Software implementation. The program, posterior to entering drug treatment of a specific patient, generate the list of possible interactions with clinical relevance, accompanied by the suggestion of the most appropriate process to be followed by the healthcare professional user of the program. In Colombia, the program have been implemented in 24 health institutions, in addition the access is free and it is available on the website http:www.udea.edu.co/pypfarmaceutica. However, further investigation to evaluate in more detail information regarding to positive predictive and negative predictive values of alert generates by program is required.

\section{Conclusions}

Due to ARV therapy is one of the most dynamic in terms of launching new products in the market, it is necessary to carry out structured and systematic review of publications on Pubmed/Medline and other electronic databases to identify new DIs in HIV-infected patients, receiving ART therapy, which must be complemented with evaluating the scientific evidence and classifying their clinical relevance.

In HIV-infected patients receiving antiretroviral therapy, closer to $80 \%$ of relevant clinical pharmacokinetic DIs are associated to induction or inhibition of the systemic hepatic metabolism, which is associated to systemic clearance for a significant number drugs. While closer to $20 \%$ of relevant clinical pharmacokinetic DIs are associated to changes in bioavailability related interactions, including changes in gastrointestinal $\mathrm{pH}$, presystemic metabolism and/or P-gp transport activity. Therefore, in HIV-infected patients receiving HAART of cART, the assessing and predicting clinical relevance of a given pharmacokinetic DI need to understand and use of concepts related to induction and, particularly with enzyme inhibition (mainly in CYP3 and CYP2 subfamilies) and, to a lesser extent, to bioavailability (especially with changes in a drug presystemic metabolism and/or P-gp transport activity).

It is becoming more evident that ARV drugs are metabolized via common pathways by CYP450 enzymatic complex, which leads to an increased probability of new clinical relevant pharmacokinetic DIs due to the inhibition or stimulation of CYP isoforms (mainly in CYP3 and CYP2 subfamilies).

In general, for a patient using more than one drug with differential capacity to modify CYP3A4 and/or P-gp enzymatic activity (some induce while some others inhibit), it is difficult to predict the net effect on the levels and effects of a drug whose metabolism is affected. (Spradling et al., 2002) Therefore, it is necessary, from a theoretical perspective, to avoid the use of such schemes, which is difficult in patients with HIV/AIDS. In this context, some studies conducted to establish the influence of several antiretroviral drugs on CYP3A activity show that ritonavir/nelfinavir inhibitory effect is maintained and it counteracts efavirenz/nevirapine inducing effect, when they are administered in combination. Additionally, it has been found that chronic administration of ritonavir ( $200 \mathrm{mg} /$ day) or nelfinavir (2.5 g/12 hours) does not increase CYP3A activity. (Fellay et al., 2005; Mouly et al., 2006)

Similar to other groups of patients, it is important to systematize, distribute, and use guidelines and recommendations based on the findings of studies indicating which 
combinations of a specified drug, of some specify therapeutic class, are the most appropriate for patients receiving cART or HAART. Such appropriate combinations should be chosen considering the lowest probability of the DIs, which should lead to a decrease in the use of less adequate combinations, as evidenced by studies in other countries. (Hulgan et al., 2005) The developing software based both on the severity of the effect, and on the probability of occurrence of a specify DI, including quality and quantity of literature that supports the interaction (evidence) in order to establish its clinical relevance, could be a notable contribution to the management of DIs in VIH-infected patients receiving ARV therapy.

Since proprietary databases and clinician assessment of severe DIs do not agree, developing a knowledge base for a DI alert system likely requires proprietary database information in conjunction with clinical opinion. (Smithburger et al., 2010) Thus, evaluation and prediction of relevant clinical DIs involves not only using DI alert system, but also the clinical interpretation of the alert and information, including drug history and the patient's clinical condition. In this task, a computer program that facilitates the evaluation, prediction, and decision on the clinical relevance of the DIs in HIV-infected patients receiving ART therapy have been designed, however its clinical utility requires be assessed in a study designed for this goal.

\section{References}

Aaron, L.; Saadoun, D.; Calatroni, I.; Launay, O.; Memain, N.; Vincent, V.; et al. (2004). Tuberculosis in HIV-infected patients: A comprehensive review. Clin Microbiol Infect, Vol. 10, pp. 388-398, ISSN 1469-0691.

Abel, S.; Jenkins, T.; Whitlock, L.; Ridgway, C. \& Muirhead, G. (2008). Effects of CYP3A4 inducers with and without CYP3A4 inhibitors on the pharmacokinetics of maraviroc in healthy volunteers. Br. J. Clin. Pharmacol, Vol. 65, No. 1, pp. 38-46, 1365-2125.

Aberg, J. (2008). Drug-drug interactions with newer antiretroviral agents. Top HIV Med, Vol.16, pp. 146-150, ISSN 15428826.

Aberg, J.; Rosenkranz, S.; Fichtenbaum, C.; Alston, B.; Brobst, S.; Segal, Y.; et al. (2006). Pharmacokinetic interaction between nelfinavir and pravastatin in HIVseronegative volunteers: ACTG Study A5108. AIDS, Vol. 20, pp. 725-729, ISSN 1473-5571.

Abrams, D.; Hilton, J. \& Leiser, R. (2003). Short-term effects of cannabinoids in patients with HIV-1 infection: A randomized, placebo-controlled clinical trial. Ann Intern Med, Vol. 139, pp. 258-266, ISSN 1539-3704.

Acosta, E. (2002). Pharmacokinetic enhancement of protease inhibitors. J Acquir Immune Defic Syndr, Vol. 29, No. 1, pp. 11-18, ISSN 1525-4135.

AIDS Patient Care. (2005). Antiviral briefs: Pregnancy category change for efavirenz. AIDS Patient Care STDS Vol. 19, pp. 413, ISSN 1557-7449.

Altice, F.; Friedland, G. \& Cooney, E. (1999). Nevirapine induced opiate withdrawal among injection drug users with HIV infection receiving methadone. AIDS, Vol. 13, pp. 957-962, ISSN 1473-5571.

Amariles, P. (2002). Interacciones medicamentosas. En: Amariles P, editor. El medicamento. Compendio básico para su utilización correcta. Medellín: Impresos LTDA, pp. 155246. 
Amariles, P. (2007). Food consumption, cytochrome P450 3A4 (CYP3A4) presystemic inhibitors, and bioavailability of saquinavir. Farm Hosp, Vol. 31, pp. 68-70, ISSN 1130-6343.

Amariles, P.; Giraldo, N. \& Faus, M. (2007a) Clinical relevance of drug interactions. Med Clin (Barc), Vol. 129, pp. 27-35, ISSN 1578-8989.

Amariles, P.; Giraldo, N. \& Faus, M. (2007b). Interacciones medicamentosas en pacientes infectados con el VIH: aproximación para establecer y evaluar su relevancia clínica. Farm Hosp. Vol. 31, pp. 283-302, ISSN 1130-6343.

Amariles, P.; Giraldo, N.; Faus, M. \& Gutiérrez, F. (2008). Proposal of a software for analysis of clinical relevance of antiretroviral drug interactions. Pharm World Sci. Vol. 30, pp. 711, ISSN 2210-7711.

Amariles, P.; Lacampa, P. \& Sáez-Benito, L. (2007c). Dosage adjustments for hepatic dysfunction Am J Health Syst Pharm, Vol. 64, No. 24, pp. 2536-2538, ISSN 1535-2900, December.

Amsay, N.; Kenny, M.; Davies, G. \& Patel, J. (2005). Complimentary and alternative medicine use among patients starting warfarin. Br J Haematol, Vol. 130, pp. 777-780, ISSN 1365-2141.

Anderson, P. \& Kakuda, T. (2006). Comment on: Suboptimal CD4 gains in HIV-infected patients receiving didanosine plus tenofovir. J Antimicrob Chemother, Vol. 58, pp. 220-221, ISSN 1460-2091.

Armstrong, S. \& Cozza, K. (2003). Pharmacokinetic drug interactions of morphine, codeine, and their derivatives: Theory and clinical reality, Part II. Psychosomatics, Vol .44, pp. 515-520, ISSN 1545-7206.

Arrington-Sanders, R.; Hutton, N.; \& Siberry, G. (2006). Ritonavir-fluticasone interaction causing Cushing syndrome in HIV-infected children and adolescents. Pediatr Infect Dis J, Vol . 25, pp. 1044-1048, ISSN 0891-3668.

Andrews, E.; Glue, P.; Fang, J.; Crownover, P.; Tressler, R. \& Damle, B. (2010). Assessment of the pharmacokinetics of co-administered maraviroc and raltegravir. $\mathrm{Br} J \mathrm{Clin}$ Pharmacol, Vol. 69, No. 1, pp. 51-57, ISSN 1365-2125, January.

Antoniou, T.; Park-Wyllie, L. \& Tseng, A. (2003). Tenofovir: A nucleotide analog for the management of human immunodeficiency virus infection. Pharmacotherapy, Vol. 23, pp. 29-43, ISSN 0277-0008.

Back, D.; Gibbons, S. \& Khoo, S. (2003). Pharmacokinetic drug interactions with nevirapine. J Acquir Immune Defic Syndr, Vol. 34, No. 1, pp. 8-14, ISSN 1077-9450.

Baker, R. (1998). Efavirenz and Fortovase. BETA. Jul:9.

Barreiro, P. \& Soriano, V. (2006). Suboptimal CD4 gains in HIV-infected patients receiving didanosine plus tenofovir. J Antimicrob Chemother, Vol. 57, pp. 806-809, ISSN 14602091.

Bays, H. (2006). Statin safety: An overview and assessment of the data-2005. Am J Cardiol, Vol. 97, No. 1, pp. 6-26, ISSN 0029149.

Becher, F.; Landman, R.; Mboup, S.; Kane, C.; Canestri, A.; Liegeois, F.; et al. (2004). Monitoring of didanosine and stavudine intracellular trisphosphorylated anabolite concentrations in HIV-infected patients. AIDS, Vol. 18, pp. 181-187, ISSN 1473-5571.

Benator, D.; Weiner, M.; Burman, W.; Vernon, A.; Zhao, Z.; Khan, A. et al. (2007). Clinical evaluation of the nelfinavir-rifabutin interaction in patients with tuberculosis and 
human immunodeficiency virus infection. Pharmacotherapy, Vol. 27, pp. 793-800, ISSN 0277-0008.

Benesic, A.; Zilly, M.; Kluge, F.; Weissbrich, B.; Winzer, R.; Klinker, H.; et al. (2004). Lipid lowering therapy with fluvastatin and pravastatin in patients with HIV infection and antiretroviral therapy: Comparison of efficacy and interaction with indinavir. Infection, Vol . 32, pp. 229-233, ISSN 0153-4453.

Bergshoeff, A.; Fraaij, P. \& Ndagijimana, J. (2005). Increased dose of lopinavir/ritonavir compensates for efavirenz-induced drug-drug interaction in HIV-1-infected children. J Acquir Immune Defic Syndr, Vol. 39, pp. 63-68, ISSN 1077-9450.

Blumberg, H.; Burman, W.; Chaisson, R.; Daley, C.; Etkind, S.; Friedman, L.; et al. (2003). American Thoracic Society/Centers for Disease Control and Prevention/Infectious Diseases Society of America: Treatment of tuberculosis. Am J Respir Crit CareMed, Vol. 167, pp. 603-662, ISSN 0003-0805.

Boffito, M.; Carriero, P. \& Trentini, L. (2002). Pharmacokinetics of saquinavir coadministered with cimetidine. J Antimicrob Chemother, Vol. 50, pp. 1081-1084, ISSN 1460-2091.

Boffito, M.; Maitland, D. \& Pozniak, A. (2006). Practical perspectives on the use of tipranavir in combination with other medications: Lessons learned from pharmacokinetic studies. J Clin Pharmacol, Vol. 46, pp. 130-139, ISSN 0091-2700.

Boffito, M.; Pozniak, A.; Kearney, B.; Higgs, C.; Mathias, A.; Zhong, L.; et al. (2005). Lack of pharmacokinetic drug interaction between tenofovir disoproxil fumarate and nelfinavir mesylate. Antimicrob Agents Chemother, Vol. 49, pp. 4386-4389, ISSN 10986569.

Bonate, P.; Reith, K. \& Weir, S. (1998). Drug interactions at the renal level. Implications for drug development. Clin Pharmacokinet, Vol. 34, pp. 375-404, ISSN 1179-1926.

Borin, M.; Chambers, J.; Carel, B.; Gagnon, S. \& Freimuth, W. (1997). Pharmacokinetic study of the interaction between rifampin and delavirdine mesylate. Clin Pharmacol Ther, Vol. 61, pp. 544-553, ISSN 1532-6535.

Boubaker, K.; Flepp, M.; Sudre, P.; Furrer, H.; Haensel, A.; Hirschel, B.; et al. (2001). Hyperlactatemia and antiretroviral therapy: The Swiss HIV Cohort Study. Clin Infect Dis Vol. 33, pp. 1931-1937, ISSN 1537-6591.

Brennan, P.; Lyus, R.; Harrison, T.; Pakianathan, M. \& Macallan, D. (2005). Pharmacokinetic interactions between efavirenz and rifampicin in the treatment of HIV and tuberculosis: One size does not fit all. AIDS, Vol. 19, pp. 1541-1543. ISSN 1473-5571.

Brophy, D.; Israel, D.; Pastor, A.; Gillotin, C.; Chittick, G.; Symonds, W.; et al. (2000). Pharmacokinetic interaction between amprenavir and clarithromycin in healthy male volunteers. Antimicrob Agents Chemother, Vol. 44, pp. 978-984, ISSN 1098-6596.

Bruce, R.; Altice, F.; Gourevitch, M. \& Friedland, G. (2006). Pharmacokinetic drug interactions between opioid agonist therapy and antiretroviral medications: Implications and management for clinical practice. J Acquir Immune Defic Syndr, Vol. 41, pp. 563-572, ISSN 1077-9450.

Burger, D.; Agarwala, S.; Child, M; Been-Tiktak, A.; Wang, Y. \& Bertz, R. (2006). Effect of rifampicin on steady-state pharmacokinetics of atazanavir with ritonavir in healthy volunteers. Antimicrob Agents Chemother, Vol. 50, pp. 3336-3342, ISSN 1098-6596. 
Caballero, J. \& Nahata, M. (2005). Use of selective serotonin-reuptake inhibitors in the treatment of depression in adults with HIV. Ann Pharmacother, Vol. 39, pp. 141-145, ISSN 0012-6667.

Cao, Y.; Smith, P. \& Wire, M. (2008). Pharmacokinetics and pharmacodynamics of methadone enantiomers after coadministration with fosamprenavir-ritonavir in opioid-dependent subjects. Pharmacotherapy, Vol. 28, pp. 863-874, ISSN 0277-0008.

Catanzaro, L.; Slish, J.; DiCenzo, R. \& Morse, G. (2004). Drug interactions with antiretrovirals. Curr Infect Dis Rep Vol. 1, pp. 89-96.

Chan, L. (2002). Drug-nutrient interaction in clinical nutrition. Curr Opin Clin Nutr Metab Care. Vol. 5, pp. 327-332, ISSN 1473-6519.

Clarke, S.; Mulcahy, F.; Tjia, J.; Reynolds, H.; Gibbons, S.; Barry, M.; et al. (2001). The pharmacokinetics of methadone in HIV-positive patients receiving the nonnucleoside reverse transcriptase inhibitor efavirenz. Br J Clin Pharmacol, Vol. 51, pp. 213-217, ISSN 1365-2125.

Coghlan, M.; Sommadossi, J.; Jhala, N.; Many, W.; Saag, M. \& Johnson, V. (2001). Symptomatic lactic acidosis in hospitalized antiretroviral treated patients with human immunodeficiency virus infection: A report of 12 cases. Clin Infect Dis, Vol. 33, pp. 1914-1921, ISSN 1537-6591.

Colombo, S.; Buclin, T.; Franc, C.; Guignard, N.; Khonkarly, M.; Tarr, P.; et al. (2006). Ritonavir-boosted atazanavir-lopinavir combination: A pharmacokinetic interaction study of total, unbound plasma and cellular exposures. Antivir Ther Vol. 11, pp. 53-62, ISSN 2040-2058.

Cooper, K.; Martin, P.; Dane, A.; Warwick, M.; Schneck, D. \& Cantarini, M. (2003). Effect of itraconazole on the pharmacokinetics of rosuvastatin. Clin Pharmacol Ther, Vol. 73, pp. 322-329, ISSN 0009-9236.

Currier, M.; Molina, G. \& Kato, M. (2004). Citalopram treatment of major depressive disorder in Hispanic HIV and AIDS patients: A prospective study. Psychosomatics, Vol. 45, pp. 210-216, ISSN 1545-7206.

Cvetkovic, R. \& Goa, K. (2003). Lopinavir/ritonavir: A review of its use in the management of HIV infection. Drugs, Vol. 63, pp. 769-802, ISSN 0012-6667.

Dagan, T.; Sable, C.; Bray, J. \& Gerschenson, M. (2002). Mitochondrial dysfunction and antiretroviral nucleoside analogue toxicities: What is the evidence? Mitochondrion Vol. 1, pp. 397-412, ISSN 1567-7249.

Dailly, E.; Allavena, C.; Raffi, F. \& Jolliet, P. (2005). Pharmacokinetic evidence for the induction of lopinavir metabolism by efavirenz. Br J Clin Pharmacol, Vol. 60, pp. 3234, ISSN 1365-2125.

Dailly, E.; Tribut, O.; Tattevin, P.; Arvieux, C.; Perré, P.; Raffi, F.; et al. (2006). Influence of tenofovir, nevirapine and efavirenz on ritonavir-boosted atazanavir pharmacokinetics in HIV-infected patients. Eur J Clin Pharmacol, Vol. 62, pp. 523526, ISSN 1432-1041.

Dalakas, M.; Semino-Mora, C. \& Leon-Monzon, M. (2001). Mitochondrial alterations with mitochondrial DNA depletion in the nerves of AIDS patients with peripheral neuropathy induced by 2'3'dideoxycytidine (ddC). Lab Invest, Vol. 81, pp. 15371544, ISSN 1530-0307.

Damle, B.; Hess, H.; Kaul, S. \& Knupp, C. (2002a). Absence of clinically relevant drug interactions following simultaneous administration of didanosine-encapsulated, 
enteric-coated bead formulation with either itraconazole or fluconazole. Biopharm Drug Dispos, Vol. 23, pp. 59-66, ISSN 0142-2782.

Damle, B.; Mummaneni, V.; Kaul, S. \& Knupp, C. (2002b). Lack of effect of simultaneously administered didanosine encapsulated enteric bead formulation (Videx EC) on oral absorption of indinavir, ketoconazole, or ciprofloxacin. Antimicrob Agents Chemother, Vol. 46, pp. 385-391, ISSN 1098-6569.

Damle, B.; Yan, J.; Behr, D.; O'Mara, E.; Nichola, P.; Kaul, S.; et al. (2002c). Effect of food on the oral bioavailability of didanosine from encapsulated enteric-coated beads. J Clin Pharmacol, Vol. 42, pp. 419-427, ISSN 0091-2700.

Daveluy, A.; Raignoux, C.; Miremont-Salamé, G.; Girodet, P.; Moore, N.; Haramburu, F.; et al. (2009). Drug interactions between inhaled corticosteroids and enzymatic inhibitors. Eur J Clin Pharmacol, Vol.65, No. 7, pp. 743-745, ISSN 1432-1041. July

Davis, J.; Schöller-Gyre, M.; Kakuda, T.; Ridgway, C.; Tweedy, S.; Ndongo, N.; et al. (2007). An open, randomized, two period, crossover study in 2 cohorts to investigate the effect of steady state TMC125 and the combination of TMC/darunavir/ritonavir on the steady state pharmacokinetics of oral maraviroc in healthy subjects. 11th European AIDS Conference. Madrid, Spain. Oct 24-27. Abstract P4.3/02.

De Jong, B.; Israelski, D.; Corbett, E. \& Small, P. (2004). Clinical management of tuberculosis in the context of HIV infection. Annu Rev Med, Vol. 55, pp. 283-301, ISSN 0066-4219.

De Maat, M.; Ekhart, G.; Huitema, A.; Koks, C.; Mulder, J. \& Beijnen J. (2003). Drug interactions between antiretroviral Drugs and comedicated agents. Clin Pharmacokinet, Vol. 42; pp. 223-282, ISSN 0312-5963.

DeJesus, E.; Piliero, P.; Summers, K.; Wire, M.; Stein, D.; Masterman, A.; et al. (2006). Interaction between fosamprenavir, with and without ritonavir, and nevirapine in human immunodeficiency virus-infected subjects. Antimicrob Agents Chemother, Vol. 50, pp. 3157-3159, ISSN 1098-6569.

DeSilva, K.; Le Flore, D.; Marston, B. \& Rimland, D. (2001). Serotonin syndrome in HIVinfected individuals receiving antiretroviral therapy and fluoxetine. AIDS, Vol .15, pp. 1281-1285, ISSN 1473-5571.

DHHS. (2005). Panel on Antiretroviral Guidelines for Adult and Adolescents. Guidelines for the use of antiretroviral agents in HIV-infected adults and adolescents. Department of Health and Human Services. In: AIDS INFO, 10.01.2011; Available from: http://www.AIDSinfo.nih.gov/contentfiles/AdultandAdolescentGL.pdf. ISBN 1588-080048

DiCenzo, R.; Peterson, D.; Cruttenden, K.; Mariuz, P.; Rezk, N.; Hochreiter, J.; et al. (2008). Effects of minocycline and valproic acid coadministration on atazanavir plasma concentrations in human immunodeficiency virus-infected adults receiving atazanavir-ritonavir. Antimicrob Agents Chemother, Vol. 52, pp. 3035-3059, ISSN 0066-4804.

Ding, R.; Tayrouz, Y.; Riedel, K.; Burhenne, J.; Weiss, J.; Mikus, G.; et al. (2004). Substantial pharmacokinetic interaction between digoxin and ritonavir in healthy volunteers. Clin Pharmacol Ther, Vol. 76, pp. 73-84, ISSN 1532-6535.

Dixit, V.; Hariparsad, N.; Li, F.; Desai, P.; Thummel, K. \& Unadkat J. (2007). Cytochrome P450 enzymes and transporters induced by antihuman immunodeficiency virus 
protease inhibitors in human hepatocytes: Implications for predicting clinical drug interactions. Drug Metab Dispos, Vol. 35, pp.1853-1859, ISSN 0090-9556.

Droste, J.; Kearney, B.; Hekster, Y. \& Burger, D. (2006). Assessment of drug-drug interactions between tenofovir disoproxil fumarate and the nonnucleoside reverse transcriptase inhibitors nevirapine and efavirenz in HIV-infected patients. J Acquir Immune Defic Syndr, Vol. 41, pp. 37-43, ISSN 1077-9450.

Droste, J.; Verweij-van, C.; Kearney, B.; Buffels, R.; Vanhorssen, P.; Hekster, Y.; et al. (2005). Pharmacokinetic study of tenofovir disoproxil fumarate combined with rifampin in healthy volunteers. Antimicrob Agents Chemother, Vol. 49, pp. 680-684, ISSN 10986569.

Etravirine: R165335, TMC 125, TMC-125, TMC125. Drugs R \& D 2006;7(6):367-73.

FDA. (1999). US Food and Drug Administration. Guidance for Industry: In vivo Drug Metabolism/Drug Interaction Studies - Study Design, Data Analysis, and Recommendations for Dosing and Labeling. Center for Drug Evaluation and Research (CDER), Center for Biologics Evaluation and Research (CBER), Rockville, MD, November 1999.

FDA. (2005). Food and Drug Administration. Title 21, Volume 5: CFR 320.33. Code of Federal Regulations. Available from:

http://www.accessdata.fda.gov/scripts/cdrh/cfdocs/cfcfr/CFRSearch.cfm?fr=32 0.24 .

Fellay, J.; Marzolini, C.; Decosterd, L.; Golay, K.; Baumann, P.; Buclin, T.; et al. (2005). Variations of CYP3A activity induced by antiretroviral treatment in HIV-1 infected patients. Eur J Clin Pharmacol, Vol. 60, pp 865-873, ISSN 1432-1041.

Fichtenbaum, C. \& Gerber J. (2002). Interactions between antiretroviral Drugs and Drugs used for the therapy of the metabolic complications encountered during HIV infection. Clin Pharmacokinet, Vol . 41, pp. 1195-1211, ISSN 84-7666-135-5.

Fichtenbaum, C.; Gerber, J.; Rosenkranz, S.; Segal, Y.; Aberg, J.; Blaschke, T.; et al. (2002). Pharmacokinetic interactions between protease inhibitors and statins in HIV seronegative volunteers: ACTG Study A5047. AIDS, Vol . 16, pp. 569-577, ISSN 1473-5571.

Finch, C.; Chrisman, C.; Baciewicz, A. \& Self, T. (2002). Rifampin and rifabutin drug interactions: An update. Arch Intern Med, Vol. 162, pp. 985-989, ISSN 1538-3679.

Fisher, M.; Talbot, G.; Maislin, G.; McKeon, B.; Tynan, K. \& Strom, B. (1989). Risk factors for amphotericin B-associated nephrotoxicity. Am J Med, Vol. 87, pp. 547-552.

Fleischer, R.; Boxwell, D. \& Sherman, K. (2004). Nucleoside analogues and mitochondrial toxicity. Clin Infect Dis, Vol. 38, pp. 79-80, ISSN 1537-6591.

Fletcher, C.; Acosta, E.; Cheng, H.; Haubrich, R.; Fischl, M.; Raasch, R.; et al. (2000). Competing drug-drug interactions among multidrug antiretroviral regimens used in the treatment of HIV- infected subjects: ACTG 884. AIDS, Vol. 14, No. 16, pp. 2495-2501, ISSN 1473-5571.

Ford, S.; Wire, M.; Lou, Y.; Baker, K. \& Stein DS. (2005). Effect of antacids and ranitidine on the single-dose pharmacokinetics of fosamprenavir. Antimicrob Agents Chemother, Vol. 49, pp. 467-469, ISSN 1098-6569.

Ford, J.; Boffito, M.; Maitland, D.; Hill, A.; Back, D.; Khoo, S.; et al. (2006). Influence of atazanavir $200 \mathrm{mg}$ on the intracellular and plasma pharmacokinetics of saquinavir 
and ritonavir 1600/100 mg administered once daily in HIV-infected patients. J Antimicrob Chemother, Vol. 58, pp. 1009-1016, ISSN 1460-2091.

Friedland, G.; Andews, L. \& Schreibman, T. (2005). Lack of an effect of atazanavir on steadystate pharmacokinetics of methadone in patients chronically treated for opiate addiction. AIDS, Vol. 19, pp. 1635-1641, ISSN 1473-5571.

Friedland, G.; Khoo, S.; Jack, C. \& Lalloo, U. (2006). Administration of efavirenz (600 $\mathrm{mg} /$ day) with rifampicin results in highly variable levels but excellent clinical outcomes in patients treated for tuberculosis and HIV. J Antimicrob Chemother, Vol. 58, pp. 1299-1302. ISSN 1460-2091.

Gaikwad, R.; Sketris, I.; Shepherd, M. \& Duffy, J. (2007). Evaluation of accuracy of drug interaction alerts triggered by two electronic medical record systems in primary healthcare. Health Informatics J, Vol. 13, pp. 163-177, ISSN 1741-2811.

Gallicano, K.; Sahai, J.; Shukla, V.; Seguin, I.; Pakuts, A.; Kwok, D.; et al. (1999). Induction of zidovudine glucuronidation and amination pathways by rifampicin in HIVinfected patients. Br J Clin Pharmacol, Vol. 48, pp. 168-179, ISSN 1365-2125.

Gerber, J.; Rosenkranz, S.; Fichtenbaum, C.; Vega, J.; Yang, A.; Alston, B.; et al. (2005). Effect of efavirenz on the pharmacokinetics of simvastatin, atorvastatin, and pravastatin: Results of AIDS Clinical Trials Group 5108 Study. J Acquir Immune Defic Syndr, Vol. 39, pp. 307-312, ISSN 1077-9450.

Giraldo, N.; Amariles, P.; Gutiérrez, F.; Monsalve, M. \& Faus, M. (2010). Aproximación para establecer y evaluar la relevancia clínica de las interacciones medicamentosas en pacientes infectados con virus de la inmunodeficiencia humana: actualización 2009. Farm Hosp, Vol. 34, pp. 90-93, ISSN 1130-6343.

Gray, D.; Roux, P.; Carrihill, M. \& Klein, M. (2010). Adrenal suppression and Cushing's syndrome secondary to ritonavir and budesonide. S Afr Med J, Vol. 100, No. 5, pp. 296-297, ISSN 2078-5131.

Grimes, R.; Lal, L. \& Lewis, S. (2002). Frequency of medical history items, drug interactions, and lifestyle characteristics that may interfere with antiretroviral medications. HIV Clin Trials, Vol. 3, pp. 161-167, ISSN 1528-4336.

Gruber, V. \& McCance-Katz, E. (2010). Methadone, buprenorphine, and street drug interactions with antiretroviral medications. Curr HIV/AIDS Rep, Vol. 7, No. 3, pp. 152-160, ISSSN 1548-3576.

Guo, Y. \& Fung, H. (2004). Fatal lactic acidosis associated with coadministration of didanosine and tenofovir disoproxil fumarate. Pharmacotherapy, Vol. 24, pp. 10891094, ISSN 0277-0008.

Hamzeh, F.; Benson, C.; Gerber, J.; Currier, J.; McCrea, J.; Deutsch, P.; et al. (2003). Steadystate pharmacokinetic interaction of modified-dose indinavir and rifabutin. Clin Pharmacol Ther Vol. 73, pp. 159-69, ISSN 1532-6535.

Hantson, P.; Di Fazio, V. \& Wallemacq, P. (2010). Toxicokinetic interaction between quetiapine and antiretroviral therapy following quetiapine overdose. Drug Metab Lett, Vol.4, No. 1, pp.7-8, ISSN 1872-3128.

Hare, C.; Vu, M.; Grunfeld, C. \& Lampiris, H. (2002). Simvastatin-nelfinavir interaction implicated in rhabdomyolysis and death. Clin Infect Dis, Vol . 35, pp. 111-112, ISSN 1058-4838.

Harris, M.; Zala, C.; Ramírez, S.; Woodfall, B.; Peeters, M.; Scholler, M.; et al. (2006). Pharmacokinetics and safety of adding TMC125 to stable regimens of saquinavir, 
lopinavir and ritonavir in HIV+adults. 13th Conference on Retroviruses and Opportunistic Infections.Denver (CO). 5-8 Feb. Abstract 575.

Havlir, D.; Tierney, C.; Friedland, G.; Pollard, R.; Smeaton, L.; Sommadossi, J.; et al. (2000). In vivo antagonism with zidovudine plus stavudine combination therapy. J Infect Dis, Vol. 182, pp. 321-325, ISSN 1537-6613.

Heck, A.; DeWitt, B. \& Lukes, A. (2000). Potential interactions between alternative therapies and warfarin. Am J Health Syst Pharm, Vol. 57, pp. 1221-1227, ISSN 1535-2900.

Ho, R. \& Kim, R. (2005). Transporters and drug therapy: implications for drug disposition and disease. Clin Pharmacol Ther, Vol. 78, pp. 260-277, ISSN 1532-6535.

Hochster, H.; Dieterich, D.; Bozzette, S.; Reichman, R.; Connor, J.; Liebes, L.; et al. (1990). Toxicity of combined ganciclovir and zidovudine for cytomegalovirus disease associated with AIDS. An AIDS Clinical Trials Group Study. Ann Intern Med, Vol. 113, pp. 111-117, ISSN 1539-3704.

Hogeland, G.; Swindells, S.; McNabb, J.; Kashuba, A.; Yee, G. \& Lindley, C. (2007). Lopinavir/ritonavir reduces bupropion plasma concentrations in healthy subjects. Clin Pharmacol Ther Vol. 81, pp. 69-75, ISSN 1532-6535.

Hoggard, P.; Sales, S.; Phiboonbanakit, D.; Lloyd, J.; Maher, B.; Khoo, S.; et al. (2001). Influence of prior exposure to zidovudine on stavudine phosphorylation in vivo and ex vivo. Antimicrob Agents Chemother, Vol. 45, pp. 577-582, ISSN 1098-6569.

Holdich, T.; Shiveley, L. \& Sawyer, J. (2007). Effect of Lamivudine on the plasma and intracellular pharmacokinetics of apricitabine, a novel nucleoside reverse transcriptase inhibitor, in healthy volunteers. Antimicrob Agents Chemother, Vol. 51. No. 8, pp. 2943-2947, ISNN 1098-6569.

Huang, L.; Wring, S.; Woolley, J.; Brouwer, K.; Serabjit-Singh, C. \& Polli, J. (2001). Induction of P-glycoprotein and cytochrome P450 3A by HIV protease inhibitors. Drug Metab Dispos Vol. 29, pp. 754-760, ISSN 0090-9556.

Hulgan, T.; Sterling, T.; Daugherty, J.; Arbogast, P.; Raffanti, S. \& Ray, W. (2005). Prescribing of contraindicated protease inhibitor and statin combinations among HIV-infected persons. J Acquir Immune Defic Syndr, Vol. 38, pp. 277-282, ISSN 1077-9450.

Hughes, C.; Freitas, A. \& Miedzinski LJ. (2007). Interaction between lopinavir / ritonavir and warfarin. CMAJ, Vol. 177, pp. 357-359, ISSN 1488-2329.

Isbister, G. \& Buckley, N. (2005). The pathophysiology of serotonin toxicity in animals and humans. Clin Neuropharmacol, Vol. 28, pp. 205-214, ISSN 0362-5664.

Izzedine, H.; Launay-Vacher, V.; Baumelou, A. \& Deray, G. (2004). Antiretroviral and immunosuppressive drug-drug interactions: An update. Kidney Int, Vol. 66, pp. 532-541, ISSN 1523-1755.

Izzo, A. (2004). Drug interactions with St. John's Wort (Hypericum perforatum): A review of the clinical evidence. Int J Clin Pharmacol Ther, Vol. 42, pp. 139-148, ISSN 01744879 .

Jacobson, T. (2004). Comparative pharmacokinetic interaction profiles of pravastatin, simvastatin, and atorvastatin when coadministered with cytochrome P450 inhibitors. Am J Cardiol, Vol . 94, pp. 1140-1146, ISSN 0029149.

Jain, A.; Venkataramanan, R.; Shapiro, R.; Scantlebury, V.; Potdar, S.; Bonham, C.; et al. (2002). The interaction between antiretroviral agents and tacrolimus in liver and kidney transplant patients. Liver Transpl. Vol. 8, pp. 841-845, ISSN 1527-6473. 
Josephson, F. (2010). Drug-drug interactions in the treatment of HIV infection: focus on pharmacokinetic enhancement through CYP3A inhibition. J Intern Med, Vol. 268, No. 6, pp. 530-539, ISSN 1365-2796, December.

Justesen, U. (2006). Therapeutic drug monitoring and human immunodeficiency virus (HIV) antiretroviral therapy. Basic Clin Pharmacol Toxicol, Vol. 98, pp. 20-31, ISSN 17427843.

Kakuda, T. \& Falcon, R. (2006). Effect of food and ranitidine on saquinavir pharmacokinetics and gastric $\mathrm{pH}$ in healthy volunteers. Pharmacotherapy Vol. 26, pp. 1060-1068, ISSN 0277-0008.

Kakuda, T.; Schöller-Gyüre, M.; Woodfall, B.; De Smedt, G.; Peeters, M.; Vandermeulen, K.; et al. (2006). TMC125 in combination with other medications: summary of drugdrug interaction studies. 8th International Congress on Drug Therapy in HIV Infection. Glasgow. 12-16 November. Oral presentation PL5.2.

Kashuba, A.; Tierney, C.; Downey, G.; Acosta, E.; Vergis, E.; Klingman, K.; et al. (2005). Combining fosamprenavir with lopinavir/ritonavir substantiallyreduces amprenavir and lopinavir exposure: ACTG protocol A5143 results. AIDS, Vol. 19, pp. 145-152, ISSN 1473-5571.

Kashuba, A. (2005). Drug-drug interactions and the Pharmacotherapy of HIV infection. Top HIV Med, Vol. 13, pp. 64-69, ISSN 1542-8826.

Katzung, B. (2009). Basic and Clinical Pharmacology. 11th ed. McGraw Hill Medical, ISBN 0071604057.

Kearney, B.; Sayre, J.; Flaherty, J.; Chen, S.; Kaul, S. \& Cheng, A. (2005). Drug-drug and drug-food interactions between tenofovir disoproxil fumarate and didanosine. $J$ Clin Pharmacol, Vol. 45, pp. 1360-1367, ISSN 0091-2700.

Khaliq, Y.; Gallicano, K., Venance, S.; Kravcik, S. \& Cameron, D. (2000). Effect of ketoconazole on ritonavir and saquinavir concentrations in plasma and cerebrospinal fluid from patients infected with human immunodeficiency virus. Clin Pharmacol Ther, Vol. 68, pp. 637-646, ISSN 1532-6535.

Kiang, T.; Ensom, M. \& Chang, T. (2005). UDP-glucuronosyltransferases and clinical drugdrug interactions. Pharmacol Ther, Vol. 106, pp. 97-132, ISSN 0163-7258.

Kiser, J.; Bumpass, J.; Meditz, A.; Anderson, P.; Bushman, L.; Ray, M.; et al. (2010). Effect of antacids on the pharmacokinetics of raltegravir in human immunodeficiency virusseronegative volunteers. Antimicrob Agents Chemother, Vol. 54, No. 12, pp. 49995003, ISSN 1098-6569.

Kraft, W.; McCrea, J.; Winchell, G.; Carides, A.; Lowry, R.; Woolf, E.; et al. (2004). Indinavir and rifabutin drug interactions in healthy volunteers. J Clin Pharmacol, Vol. 44, pp. 305-313, ISSN 1552-4604.

Krikorian, S. \& Rudorf, D. (2005). Drug-drug interactions and HIV therapy: What should pharmacists know? J Pharm Pract, Vol. 18, pp. 278-294, ISSN 0897-1900.

La Porte, C.; Colbers, E.; Bertz, R.; Voncken, D.; Wikstrom, K.; Boeree, M.; et al. (2004). Pharmacokinetics of adjusted-dose lopinavir-ritonavir combined with rifampin in healthy volunteers. Antimicrob Agents Chemother, Vol. 48, pp. 1553-1560, ISSN 10986596.

La Porte, C.; Verweij-van, C.; van Ewijk, N.; Aarnoutse, R.; Koopmans, P.; Reiss, P.; et al. (2005). Pharmacokinetic interaction study of indinavir/ritonavir and the enteric- 
coated capsule formulation of didanosine in healthy volunteers. J Clin Pharmacol, Vol. 45, pp. 211-218, ISSN 0091-2700.

Laganiere, S.; Davies, R.; Carignan, G.; Foris, K.; Goernert, L.; Carrier, K.; et al. (1996). Pharmacokinetic and pharmacodynamic interactions between diltiazem and quinidine. Clin Pharmacol Ther, Vol. 60, pp. 255-264, ISSN 1532-6535.

Launay, V.; Izzedine, H.; Karie, S.; Hulot, J.; Baumelou, A. \& Deray, G. (2006). Renal tubular drug transporters. Nephron Physiol, Vol. 103, pp. 97-106, ISSN 1660-2137.

Lee, L.; Andrade, A. \& Flexner, C. (2006). Interactions between natural health products and antiretroviral Drugs: Pharmacokinetic and pharmacodynamic effects. Clin Infect Dis Vol. 43, pp. 1052-1059, ISSN 1537-6591.

Levêque, D.; Santucci, R.; Pavillet, J.; Herbrecht, R. \& Bergerat J. (2009). Paralytic ileus possibly associated with interaction between ritonavir/lopinavir and vincristine. Pharm World Sci, Vol. 31, No. 6, pp. 619-621, ISSN 2210-7711.

Lim, M.; Min, S.; Eron, J.; Bertz, R.; Robinson, M.; Gaedigk, A.; et al. (2004). Coadministration of lopinavir/ritonavir and phenytoin results in twoway drug interaction through cytochrome P-450 induction. J Acquir Immune Defic Syndr. Vol. 36, pp. 1034-40, ISSN 1077-9450.

Lin, J. \& Yamazaki, M. (2003). Role of P-glycoprotein in pharmacokinetics: Clinical implications. Clin Pharmacokinet, Vol. 42, pp. 59-98, ISSN 0312-5963.

Lin, J. (2003). Drug-drug interaction mediated by inhibition and induction of P-glycoprotein. Adv Drug Deliv Rev, vol. 55, pp. 53-81, ISSN 0169-4092.

Maddox, R.; Groves, W. \& Ensom, R. (1980). Automated pharmaceutical assistance systems. I. Clinical pharmacokinetics. II. Drug interference with laboratory tests results. J Med Syst, Vol. 4, pp. 151-168, ISSN 0148-5598.

Makinson, A.; Pujol, J.; Le Moing, V.; Peyriere, H. \& Reynes J. (2010). Interactions between cytotoxic chemotherapy and antiretroviral treatment in human immunodeficiency virus-infected patients with lung cancer. J Thorac Oncol, Vol. 5, No. 4, pp. 562-571, ISSN 1556-1380.

Manosuthi, W.; Sungkanuparph, S.; Thakkinstian, A.; Vibhagool, A.; Kiertiburanakul, S.; Rattanasiri, S.; et al. (2005). Efavirenz levels and 24- week efficacy in HIV-infected patients with tuberculosis receiving highly active antiretroviral therapy and rifampicin. AIDS, Vol. 19, pp. 1481-1486, ISSN 1473-5571.

Manosuthi, W.; Kiertiburanakul, S.; Sungkanuparph, S.; Ruxrungtham, K.; Vibhagool, A.: Rattanasiri, S.; et al. (2006). Efavirenz $600 \mathrm{mg} /$ day versus efavirenz $800 \mathrm{mg} /$ day in HIV-infected patients with tuberculosis receiving rifampicin: 48 weeks results. AIDS , Vol. 20, pp. 131-132, ISSN 1473-5571.

Markowitz, J. \& DeVane, C. (2001). The emerging recognition of herb-drug interactions with a focus on St. John's wort (Hypericum perforatum). Psychopharmacol Bull. Vol. 35, pp. 53-64.

Martinez, E.; Milinkovic, A.; de Lazzari, E.; Ravasi, G.; Blanco, J.; Larrousse, M:; et al. (2004). Pancreatic toxic effects associated with co-administration of didanosine and tenofovir in HIV-infected adults. Lancet, Vol. 364, pp. 65-67, ISSN 0140-6736.

Masía, M.; Gutiérrez, F.; Padilla, S.; Ramos, J. \& Pascual, J. (2005). Severe toxicity associated with the combination of tenofovir and didanosine: Case report and review. Int $J$ STD AIDS Vol. 16, pp. 646-648, ISSN 0956-4624. 
Matteelli, A.; Regazzi, M.; Villani, P.; De Iaco, G.; Cusato, M.; Carvalho, A.; et al. (2007). Multiple-dose pharmacokinetics of efavirenz with and without the use of rifampicin in HIV-positive patients. Curr HIV Res, Vol. 5, pp. 349-353, ISSN 15701621.

Mauss, S.; Scholten, S.; Wolf, E.; Berger, F.; Schmutz, G.; Jaeger, H.; et al. (2004). A prospective, controlled study assessing the effect of lopinavir on amprenavir concentrations boosted by ritonavir. HIV Med, Vol. 5, pp. 15-17, ISSN ISSN 15428826.

McCance-Katz, E.; Moody, D. \& Morse, G. (2006). Interactions between buprenorphine and antiretrovirals I: Non-nucleoside reverse transcriptase inhibitors: Efavirenz and delavirdine. Clin Infect Dis, Vol. 43, pp. S224-S234, ISSN 1537-6591.

McCance-Katz, E.; Rainey, P. \& Friedland G. (2003). The protease inhibitor lopinavir/ritonavir may produce opiate withdrawal in methadone-maintained patients. Clin Infect Dis, Vol. 37, pp. 476-482, ISSN 1537-6591.

McCance-Katz, E.; Rainey, P. \& Smith, P. (2004). Drug interactions between opioide and antiretroviral medications: Interaction between methadone, LAAM and nelfinavir. Am J Addict, Vol.13, pp.163-180, ISSN 1521-0391.

Menard, A.; Solas, C.; Mokthari, S; Bregigeon, S.; Drogoul, M.; Tamalet, C.; et al. (2009). Etravirine- raltegravir, a marked interaction in HIV-1 infected patients: about four cases. AIDS, Vol. 23, pp. 869-871, ISSN 1473-5571.

Mildvan, D.; Yarrish, R.; Marshak, A.; Hutman, H.; McDonough, M.; Lamson, M.; et al. (2002). Pharmacokinetic interaction between nevirapine and ethinyl estradiol/norethindrone when administered concurrently to HIV-infected women. J Acquir Immune Defic Syndr, Vol. 29, pp. 471-477, ISNN 1077-9450.

Miller, C.; El-Kholi, R-; Faragon, J. \& Lodise, T. (2007). Prevalence and risk factors for clinically significant drug interactions with antiretroviral therapy. Pharmacotherapy, Vol. 27, No. 10, pp. 1379-1386, ISSN 0277-0008.

Moling, O.; Rimenti, G.; Pristerà, R.; Vedovelli, C.; Pagani, L.; Spoladore, G.; et al. (2009). Fatal lactic acidosis precipitated by nifedipine in a patient treated with disulfiram and antiretrovirals. Drug Metab Lett. Vol. 3, No. 3, pp. 176-180, ISSN 1872-3128.

Mouly, S.; Rizzo-Padoin, N.; Simoneau, G.; Verstuyft, C.; Aymard, G.; Salvat, C.; et al. (2006). Effect of widely used combinations of antiretroviral therapy on liver CYP3A4 activity in HIV-infected patients. Br J Clin Pharmacol, Vol. 62, pp. 200-209, ISSN 1365-2125.

Mullin, P.; Green, G. \& Bakshi, R. (2004). Special populations: The management of seizures in HIV-positive patients. Curr Neurol Neurosci Rep, Vol.4, pp.308-14, ISSN: 15346293.

Nettles, R.; Kieffer, T.; Parsons, T.; Johnson, J.; Cofrancesco, J.; Gallant, J.; et al. (2006). Marked intraindividual variability in antiretroviral concentrations may limit the utility of therapeutic drug monitoring. Clin Infect Dis, Vol. 42, pp. 1189-1196, ISSN 1537-6591.

Obach, R.; Walsky, R.; Venkatakrishnan, K.; Gaman, E.; Houston, J. \& Tremaine, L. (2006). The utility of in vitro cytochrome P450 inhibition data in the prediction of drugdrug interactions. Pharmacol Exp Ther, Vol. 316, pp. 336-348, ISSN 1521-0103.

Obach, R.; Walsky, R.; Venkatakrishnan, K.; Houston, J. \& Tremaine, L. (2005) In vitro cytochrome P450 inhibition data and the prediction of drug-drug interactions: 
qualitative relationships, quantitative predictions, and the rank-order approach. Clin Pharmacol Ther, Vol. 78, pp. 582-592, ISSN 1532-6535.

Oette, M.; Kurowski, M.; Feldt, T.; Kroidl, A.; Sagir, A.; Vogt, C.; et al. (2005). Impact of rosiglitazone treatment on the bioavailability of antiretroviral compounds in HIV-positive patients. J Antimicrob Chemother, Vol. 56, pp. 416-419, ISSN 14602091.

Ogbuokiri, J. (2009). Optimizing care for patients on maraviroc salvage therapy. HIV Clin Summer, Vol. 21, No. 3, pp. 11-15, ISSN 1551-8850

Oldfield, V. \& Plosker, G. (2006). Lopinavir/ritonavir: A review of its use in the management of HIV infection. Drugs, Vol. 66, pp. 1275-1299, ISSN 0012-6667.

Ouellet, D.; Hsu, A.; Qian, J.; Locke, C.; Eason, C.; Cavanaugh, J.; et al. (1998). Effect of ritonavir on the pharmacokinetics of ethinyl estradiol in healthy female volunteers. Br J Clin Pharmacol, Vol. 46, pp. 111-116, ISSN 1365-2125.

Pau, A. \& Boyd, S. (2010). Recognition and management of significant drug interactions in HIV patients: challenges in using available data to guide therapy. Clin Pharmacol Ther, Vol. 88, No. 5, pp. 712-719, ISSN 1532-6535.

Penzak, S.; Shen, J.; Alfaro, R.; Remaley, A.; Natarajan, V. \& Falloon J. (2004). Ritonavir decreases the nonrenal clearance of digoxin in healthy volunteers with known MDR1 genotypes. Ther Drug Monit, Vol. 26, pp. 322-330, ISSN 1536-3694.

Pérez, V.; Sánchez, P. \& Villar, S. (2009). Etravirine drug interactions. Enferm Infecc Microbiol Clin, Vol. 27, No. 2, pp. 27-31, ISSN 1578-1852.

Perronne, C. (2006). Antiviral hepatitis and antiretroviral drug interactions. J Hepatol, Vol. 44, No. 1, pp. 119-125.

Petit, F.; Fromenty, B.; Owen, A. \& Estaquier, J. (2005). Mitochondria are sensors for HIV Drugs. Trends Pharmacol Sci, Vol. 26, pp. 258-264, ISSN 0165-6147.

Piscitelli, S \& Gallicano, K. (2001). Interactions among Drugs for HIV and opportunistic infections. N Engl Med, Vol . 344, pp. 984-996, ISSN 0028-4793.

Polk, R.; Brophy, D.; Israel, D.; Patron, R.; Sadler, B.; Chittick; G.; et al. (2001). Pharmacokinetic interaction between amprenavir and rifabutin or rifampin in healthy males. Antimicrob Agents Chemother, Vol. 45, pp. 502-508, ISSN 1098-6596.

Polk, R.; Crouch, M.; Israel, D.; Pastor, A.; Sadler, B.; Chittick, G.; et al. (1999). Pharmacokinetic interaction between ketoconazole and amprenavir after single doses in healthy men. Pharmacotherapy, Vol.19, pp. 1378-1384, ISSN 0277-0008.

Ramachandran, G.; Hemanthkumar, A.; Rajasekaran, S.; Padmapriyadarsini, C.; Narendran, G.; Sukumar, B.; et al. (2006). Increasing nevirapine dose can overcome reduced bioavailability due to rifampicin coadministration. J Acquir Immune Defic Syndr, Vol. 42, pp. 36-41. ISSN 1077-9450.

Rathbun, R. \& Rossi, D. (2002). Low-dose ritonavir for protease inhibitor pharmacokinetic enhancement. Ann Pharmacother Vol. 36, pp. 702-706, ISSN 0012-6667.

Ray, A.; Olson, L. \& Fridland, A. (2004). Role of purine nucleoside phosphorylase in interactions between $2^{\prime}, 3^{\prime}$-dideoxyinosine and allopurinol, ganciclovir, or tenofovir. Antimicrob Agents Chemother Vol. 48, pp. 1089-1095, ISSN 1098-6569.

Ray, A. (2005). Intracellular interactions between nucleos(t)ide inhibitors of HIV reverse transcriptase. AIDS Rev, Vol. 7, pp. 113-125. 
Ray, W.; Murray, K.; Meredith, S.; Narasimhulu, S.; Hall, K. \& Stein, C. (2004). Oral erythromycin and the risk of sudden death from cardiac causes. $N$ Engl J Med, Vol. 351, pp. 1089-1096, ISSN 1533-4406.

Ribera, E.; Azuaje, C.; López, R.; Díaz, M.; Feijoo, M.; Pou, L.; et al. (2006). Atazanavir and lopinavir/ritonavir: Pharmacokinetics, safety and efficacy of a promising doubleboosted protease inhibitor regimen. AIDS, Vol. 20, pp. 1131-1139, ISSN 1473-5571.

Ribera, E.; Azuaje, C.; López, R.; Domingo, P.; Curran, A.; Feijoo, M.; et al. (2007). Pharmacokinetic interaction between rifampicin and the once-daily combination of saquinavir and low-dose ritonavir in HIV-infected patients with tuberculosis. $J$ Antimicrob Chemother, Vol. 59, pp. 690-697, ISSN 1460-2091.

Ribera, E.; Pou, L.; López, R.; Crespo, M.; Falco, V.; Ocaña, I.; et al. (2001). Pharmacokinetic interaction between nevirapine and rifampicin in HIVinfected patients with tuberculosis. J Acquir Immune Defic Syndr, Vol. 28, pp. 450-453, ISSN 1077-9450.

Robertson, S.; Penzak, S. \& Pau, A. (2005). Drug interactions in the management of HIV infection. Expert Opin Pharmacother, Vol. 6, pp. 233-253, ISSN 1744-7666.

Rodríguez, A.; Caraballo, M.; Palma, D.; Santos, B.; Molina, T.; Desongles, T.; et al. (2009). Quality of interaction database management systems. Farm Hosp, Vol. 33, pp. 134146, ISSN 1130-6343.

Rolla, V.; da Silva Vieira, M.; Pereira, P.; Lourenço, M.; de Jesus, C.; Gonçalves, M.; et al. (2006). Safety, efficacy and pharmacokinetics of ritonavir $400 \mathrm{mg} /$ saquinavir 400 mg twice daily plus rifampicin combined therapy in HIV patients with tuberculosis. Clin Drug Investig, Vol. 26, pp. 469-479, ISSN 1179-1918.

Romanelli, F. \& Pomeroy, C. (2003). Concurrent use of antiretrovirals and anticonvulsants in human immunodeficiency virus (HIV) seropositive patients. Curr Pharm Des, Vol.9, pp. 1433-1439, ISSN 1381-6128.

Santos, C. \& Boullata, J. (2005). An approach to evaluating drug-nutrient interactions. Pharmacotherapy. Vol. 25, pp. 1789-1800, ISSN 0277-0008.

Saraga, M.; Preisig, M. \& Zullino, D. (2006). Reduced valproate plasma levels possible after introduction of efavirenz in a bipolar patient. Bipolar Disord, Vol. 8, pp. 415-417, ISSN 1399-5618.

Sax, P. (2006). Strategies for management and treatment of dyslipidemia in HIV/AIDS. AIDS Care, Vol . 18, pp. 149-157, ISSN 0954-0121.

Schmidt, G.; Hoehns, J.; Purcell, J.; Friedman, R. \& Elhawi, Y. (2007). Severe rhabdomyolysis and acute renal failure secondary to concomitant use of simvastatin, amiodarone, and atazanavir. J Am Board Fam Med, Vol. 20, pp. 411-416, ISSN 1558-7118.

Schöller, M.; Woodffall, B.; De Marez, T.; De Smedt, G.; Peeters, M.; Vandermeulen, K.; et al. (2006). Pharmacokinetics of TMC125 with atazanavir and atazanavir/ritonavir. 8th Internacional Congress on Drug Therapy in HIV Infection. Glasgow. Nov 12-16, Abstract P278.

Schöller-Gyüre, M.; Kakuda, T.; De Smedt, G.; Vanaken, H.; Bouche, M.; Peeters, M.; et al. (2008). A pharmacokinetic study of etravirine (TMC125) co-administered with ranitidine and omeprazole in HIV-negative volunteers. Br J Pharmacol, Vol. 66, pp. 508-516, ISSN 1476-5381.

Schöller-Gyüre, M.; Kakuda, T.; De Smedt, G.; Woodfall, B.; Bollen, S.; Peeters, M.; et al. (2007). Pharmacokinetic interaction between the non-nucleoside reverse transcriptase inhibitor TMC125 and atorvastatin in HIV-negative volunteers. 4th 
International AIDS Society Conference on HIV Pathogenesis, Treatment and Prevention. Sydney. 22-25 Jul. Abstract WEPEA 106.

Schöller-Gyüre, M.; Woodfall, B.; Debroye, C.; De Marez, T.; Peeters, M.; Vandermeulen, K.; et al. (2006). Pharmacokinetic interaction between TMC125 and rifabutin. 44th Annual Meeting of the Infectious Diseases Society of America. Toronto. 12-15 Oct. Abstract 963

Sekar, V.; De Pauw, M. \& Mariën, K. (2007a). Pharmacokinetic interaction between TMC114/r and efavirenz in healthy volunteers. Antivir Ther, Vol. 12, No. 4, pp. 509-514, ISSN 2040-2058.

Sekar, V.; Lefebvre, E. \& Boogaerts, G. (2006a). Pharmacokinetic interaction between the protease inhibitors TMC114 and lopinavir/ritonavir. 46th Interscience Conference on Antimicrobial Agents and Chemotherapy, Sep 27-30, San Francisco (CA), abstract no. A-367.

Sekar, V.; Lefebvre, E.; De Marez, T.; De Pauw, M.; De Paepe, E.; Vangeneugden, T.; et al. (2008). Effect of repeated doses of darunavir plus low-dose ritonavir on the pharmacokinetics of sildenafil in healthy male subjects: phase I randomized, openlabel, two-way crossover study. Clin Drug Investig, Vol. 28, No. 84, pp. 79-85, ISSN 1179-1918.

Sekar, V.; Lefebvre, E. \& De Paepe, E.; et al. (2007b). Pharmacokinetic interaction between darunavir boosted with ritonavir and omeprazole or ranitidine in human immunodeficiency virus-negative healthy volunteers. Antimicrob Agents Chemother, Vol. 51, No. 3, pp. 958-961, ISSN 1098-6569, March.

Sekar, V.; Lefebvre, E. \& Mariën, K. (2006b). Pharmacokinetic interaction between the HIV protease inhibitors TMC114 and saquinavir, in the presence of low-dose ritonavir. 44th Annual Meeting of the Infectious Disease Society of America, Oct 12-15; Toronto (ON). abstract no. 959.

Sekar, V.; Lavreys, L; Van de Casteele, T; Berckmans, C.; Guzman, S.; Vangeneugden, T.; et al. (2010). Pharmacokinetics of darunavir/ritonavir and rifabutin coadministered in HIV-negative healthy volunteers. Antimicrob Agents Chemother, Vol. 54, No. 10, pp. 4440-4445, ISSN 1098-6569.

Setzer, B.; Lebrecht, D. \& Walker, U. (2008). Pyrimidine nucleoside depletion sensitizes to the mitochondrial hepatotoxicity of the reverse transcriptase inhibitor stavudine. Am J Pathol. Vol. 172, No. 3, pp. 681-690.

Shriner, K. \& Goetz, M. (1992). Severe hepatotoxicity in a patient receiving both acetaminophen and zidovudine. Am J Med, Vol. 93, pp. 94-96.

Simpson, D. \& Tagliati, M. (1995). Nucleoside analogue associated peripheral neuropathy in human immunodeficiency virus infection. I Acquir Immune Defic Syndr Hum Retrovirol, Vol. 9, pp. 153-161.

Smithburger, P.; Kane-Gill, S.; Benedict, N.; Falcione, B. \& Seybert, A. (2010). Grading the severity of drug-drug interactions in the intensive care unit: a comparison between clinician assessment and proprietary database severity rankings. Ann Pharmacother. Vol. 44, No. 11, pp. 1718-1724, ISSN 0012-6667.

Solas, C.; Poizot-Martin, I.; Drogoul, M.; Ravaux, I.; Dhiver, C.; Lafeuillade, A.; et al. (2004). Therapeutic drug monitoring of lopinavir/ritonavir given alone or with a nonnucleoside reverse transcriptase inhibitor. Br J Clin Pharmacol, Vol. 57, pp. 436-440, ISSN 1365-2125. 
Spradling, P.; Drociuk, D.; McLaughlin, S.; Lee, L.; Peloquin, C.; Gallicano, K.; et al. (2002). Drug-drug interactions in inmates treated for human immunodeficiency virus and Mycobacterium tuberculosis infection or disease: An institutional tuberculosis outbreak. Clin Infect Dis, Vol. 35, pp. 1106-1112, ISSN 1537-6591.

Steele, M.; Burk, R. \& DesPrez, R. (1991). Toxic hepatitis with isoniazide and rifampin: A meta-analysis. Chest, Vol. 99, pp. 465-71, ISSN 1931-3543.

Stocker, H.; Kruse, G. \& Kreckel, P. (2004). Nevirapine significantly reduces the levels of racemic methadone and (R)-methadone in human immunodeficiency virus-infected patients. Antimicrob Agents Chemother, Vol. 48, pp. 4148-4153, ISSN 1098-6569.

Sudano, I.; Spieker, L.; Noll, G.; Corti, R.; Weber, R. \& Luscher, T. (2006). Cardiovascular disease in HIV infection. Am Heart J, Vol . 151, pp. 1147-1155, ISSN 0002-8703.

Taburet, A.; Piketty, C.; Chazallon, C.; Vincent, I.; Gerard, L.; Calvez, V:; et al. (2004). Interactions between atazanavir-ritonavir and tenofovir in heavily pretreated human immunodeficiency virus-infected patients. Antimicrob Agents Chemother, Vol. 48, pp. 2091-2096, ISSN 1098-6569.

Taburet, A.; Raguin, G.; Le Tiec, C.; Droz, C.; Barrail, A.; Vincent, I.; et al. (2004). Interactions between amprenavir and the lopinavir-ritonavir combination in heavily pretreated patients infected with human immunodeficiency virus. Clin Pharmacol Ther, Vol 75, pp. 310-323, ISSN 1532-6535.

Tibotec (2008). Prezista ${ }^{\circledR}$ (darunavir) tablet: US prescribing information. Raritan (NJ), 25.03.2009 Available from:

http://www.prezista.com/prezista/documents/us_package_insert.pdf

Tomilo, D.; Smith, P.; Ogundele, A.; Difrancesco, R.; Berenson, C.; Eberhardt, E.; et al. (2006). Inhibition of atazanavir oral absorption by lansoprazole gastric acid suppression in healthy volunteers. Pharmacotherapy, vol. 26, pp. 341-436, ISSN 0277-0008.

Tong, L.; Phan, T.; Robinson, K.; Babusis, D.; Strab, R.; Bhoopathy, S.; et al. (2007). Effects of human immunodeficiency virus protease inhibitors on the intestinal absorption of tenofovir disoproxil fumarate in vitro. Antimicrob Agents Chemother, Vol.51, pp. 3498-3504, ISSN 1098-6596.

Touzot, M.; Beller, C.; Touzot, F.; Louet, A. \& Piketty, C. (2006). Dramatic interaction between levothyroxine and lopinavir/ritonavir in a HIV-infected patient. AIDS, Vol. 20, pp. 1210-1212, ISSN 1473-5571.

Tran, J.; Gerber, J. \& Kerr, B. (2001). Delavirdine: Clinical pharmacokinetics and drug interactions. Clin Pharmacokinet, Vol. 40, pp. 207-226, ISSN 0312-5963.

Tran, J.; Petersen, C.; Garrett, M.; Hee, B. \& Kerr, B. (2002). Pharmacokinetic interaction between amprenavir and delavirdine: Evidence of induced clearance by amprenavir. Clin Pharmacol Ther, Vol. 72, pp. 615-626, ISSN 1532-6535.

Tseng, A.; Nguyen, M.; Cardella, C.; Humar, A \& Conly, J. (2002). Probable interaction between efavirenz and cyclosporine. AIDS, Vol. 16, pp. 505-506, ISSN 1473-5571.

Tseng, A. \& Foisy M. (1999). Significant interactions with new antiretrovirals and psychotropic Drugs. Ann Pharmacother, Vol. 33, pp. 461-473, ISSN 0012-6667.

Tseng, A. \& Salit, I. (2007). CD4+ cell count decline despite HIV suppression: A probable didanosine-valganciclovir interaction. Ann Pharmacother, Vol. 41, pp. 512-517, ISSN 0012-6667. 
Tucker, G.; Houston, J. \& Huang, S. (2001). Optimizing drug development: strategies to assess drug metabolism/transporter interaction potential-toward a consensus. Clin Pharmacol Ther, Vol. 70, pp. 103-114, ISSN 1532-6535.

Van der Lee, M.; Dawood, L.; Hofstede, H.; Graaff-Teulen, M.; Ewijk-Beneken, Caliskan, N.; et al. (2006). Lopinavir/ritonavir reduces lamotrigine plasma concentrations in healthy subjects. Clin Pharmacol Ther, Vol. 80, pp. 159-168, ISSN 1532-6535.

Vogel, M.; Voigt, E.; Michaelis, H.; Sudhop, T.; Wolff, M.; Turler, A.; et al. (2004). Management of drug-to-drug interactions between cyclosporine $\mathrm{A}$ and the protease-inhibitor lopinavir/ritonavir in liver-transplanted HIV-infected patients. Liver Transpl, Vol 10, pp. 939-944, ISSN 1524-6473.

Von, L.; Greenblatt, D.; Duan, S.; Daily, J.; Harmatz, J. \& Shader, R. (1998). Inhibition of desipramine hydroxylation (Cytochrome P450-2D6) in vitro by quinidine and by viral protease inhibitors: Relation to drug interactions in vivo. J Pharm Sci, Vol. 87, pp. 1184-1189, ISSN 1520-6017.

Vourvahis, M. \& Kashuba, A. (2007). Mechanisms of pharmacokinetic and pharmacodynamic drug interactions associated with ritonavirenhanced tipranavir. Pharmacotherapy, Vol. 27, pp.888-909, ISSN 0277-0008.

Weiner, M.; Benator, D.; Peloquin, C.; Burman, W.; Vernon, A.; Engle, M.; et al. (2005). Evaluation of the drug interaction between rifabutin and efavirenz in patients with HIV infection and tuberculosis. Clin Infect Dis,Vol.41, pp. 1343-1349. ISSN 15376591.

Wertheimer, B.; Freedberg, K.; Walensky, R.; Yazdanapah, Y. \& Losina, E. (2006). Therapeutic drug monitoring in HIV treatment: A literature review. HIV Clin Trials, Vol. 7, pp. 59-69, ISSN 1528-4336.

Winston, A.; Back, D.; Fletcher, C.; Robinson, L.; Unsworth, J.; Tolowinska, I.; et al. (2006). Effect of omeprazole on the pharmacokinetics of saquinavir-500 mg formulation with ritonavir in healthy male and female volunteers. AIDS, Vol. 20, pp. 1401-1406, ISSN 1473-5571.

Winston, A. \& Boffito, M. (2005). The management of HIV-1 protease inhibitor pharmacokinetic interactions. J Antimicrob Chemother, Vol . 56, pp. 1- 5, ISSN 03057453.

Wire, M.; Shelton, M. \& Studenberg S. (2006). Fosamprenavir: Clinical pharmacokinetics and drug interactions of the amprenavir prodrug. Clin Pharmacokinet, Vol . 45, pp. 137168, ISSN 0312-5963.

Wittkop, L.; Breilh, D.; DaSilva, D.; Duffau, P.; Mercié, P.; Raymond, I.; et al. (2009). Virological and immunological response in HIV-1-infected patients with multiple treatment failures receiving raltegravir and optimized background therapy, ANRS CO3 Aquitaine Cohort. J Antimicrob Chemother, Vol. 63, 1251-1255, ISSN 1460-2091.

Yee, D.; Valiquette, C.; Pelletier, M.; Parisien, I.; Rocher, I. \& Menzies, D. (2003). Incidence of serious side effects from first-line antituberculosis Drugs among patients treated for active tuberculosis. Am J Respir Crit Care Med, Vol. 167, pp. 1472-1477.

Young, B. (2005). Review: Mixing new cocktails: Drug interactions in antiretroviral regimens. AIDS Patient Care STDS, Vol. 19, pp. 286-297, ISSN 1557-7449. 
Zeldin, R. \& Petruschke, R. (2004). Pharmacological and therapeutic properties of ritonavirboosted protease inhibitor therapy in HIV-infected patients. J Antimicrob Chemother, Vol. 53, pp. 4-9, ISSN 1460-2091.

Zhou, S.; Chan, E.; Pan, S.; Huang, M. \& Lee, E. (2004). Pharmacokinetic interactions of Drugs with St John's wort. J Psychopharmacol, Vol. 18, pp. 262-276, ISSN 1461-7285. 


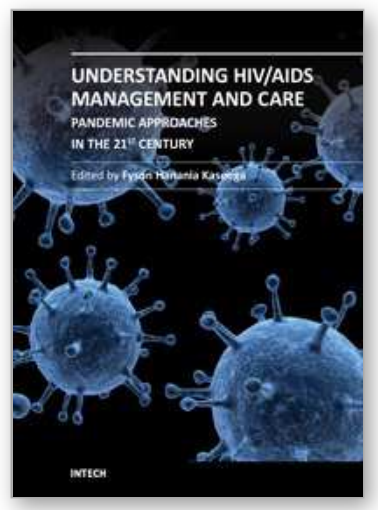

\author{
Understanding HIV/AIDS Management and Care - Pandemic \\ Approaches in the 21st Century \\ Edited by Dr. Fyson Kasenga
}

ISBN 978-953-307-603-4

Hard cover, 384 pages

Publisher InTech

Published online 14, December, 2011

Published in print edition December, 2011

Like any other book on the subject of HIV/AIDS, this book is not a substitute or exhausting the subject in question. It aims at complementing what is already in circulation and adds value to clarification of certain concepts to create more room for reasoning and being part of the solution to this global pandemic. It is further expected to complement a wide range of studies done on this subject, and provide a platform for the more updated information on this subject. It is the hope of the authors that the book will provide the readers with more knowledge and skills to do more to reduce HIV transmission and improve the quality of life of those that are infected or affected by HIV/AIDS.

\title{
How to reference
}

In order to correctly reference this scholarly work, feel free to copy and paste the following:

Pedro Amariles, Newar Giraldo Alzate and Maria Jose Faus (2011). Clinical Relevance of Drug Interactions in HIV-Infected Patients Receiving Antiretroviral Therapy, Understanding HIV/AIDS Management and Care Pandemic Approaches in the 21st Century, Dr. Fyson Kasenga (Ed.), ISBN: 978-953-307-603-4, InTech, Available from: http://www.intechopen.com/books/understanding-hiv-aids-management-and-care-pandemicapproaches-in-the-21st-century/clinical-relevance-of-drug-interactions-in-hiv-infected-patients-receivingantiretroviral-therapy

\section{INTECH}

open science | open minds

\author{
InTech Europe \\ University Campus STeP Ri \\ Slavka Krautzeka 83/A \\ 51000 Rijeka, Croatia \\ Phone: +385 (51) 770447 \\ Fax: +385 (51) 686166 \\ www.intechopen.com
}

\author{
InTech China \\ Unit 405, Office Block, Hotel Equatorial Shanghai \\ No.65, Yan An Road (West), Shanghai, 200040, China \\ 中国上海市延安西路65号上海国际贵都大饭店办公楼 405 单元 \\ Phone: +86-21-62489820 \\ Fax: +86-21-62489821
}


(C) 2011 The Author(s). Licensee IntechOpen. This is an open access article distributed under the terms of the Creative Commons Attribution 3.0 License, which permits unrestricted use, distribution, and reproduction in any medium, provided the original work is properly cited. 